\title{
Search for direct production of electroweakinos in final states with missing transverse momentum and a Higgs boson decaying into photons in pp collisions at $\sqrt{\mathrm{s}}=13 \mathrm{TeV}$ with the ATLAS detector
}

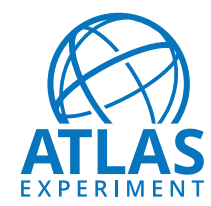

\section{The ATLAS collaboration}

E-mail: atlas.publications@cern.ch

ABSTRACT: A search for a chargino-neutralino pair decaying via the $125 \mathrm{GeV}$ Higgs boson into photons is presented. The study is based on the data collected between 2015 and 2018 with the ATLAS detector at the LHC, corresponding to an integrated luminosity of $139 \mathrm{fb}^{-1}$ of $p p$ collisions at a centre-of-mass energy of $13 \mathrm{TeV}$. No significant excess over the expected background is observed. Upper limits at $95 \%$ confidence level for a massless $\tilde{\chi}_{1}^{0}$ are set on several electroweakino production cross-sections and the visible cross-section for beyond the Standard Model processes. In the context of simplified supersymmetric models, $95 \%$ confidence-level limits of up to $310 \mathrm{GeV}$ in $m\left(\tilde{\chi}_{1}^{ \pm} / \tilde{\chi}_{2}^{0}\right)$, where $m\left(\tilde{\chi}_{1}^{0}\right)=0.5 \mathrm{GeV}$, are set. Limits at $95 \%$ confidence level are also set on the $\tilde{\chi}_{1}^{ \pm} \tilde{\chi}_{2}^{0}$ cross-section in the mass plane of $m\left(\tilde{\chi}_{1}^{ \pm} / \tilde{\chi}_{2}^{0}\right)$ and $m\left(\tilde{\chi}_{1}^{0}\right)$, and on scenarios with gravitino as the lightest supersymmetric particle. Upper limits at the $95 \%$ confidence-level are set on the higgsino production crosssection. Higgsino masses below $380 \mathrm{GeV}$ are excluded for the case of the higgsino fully decaying into a Higgs boson and a gravitino.

KEYWORDS: Hadron-Hadron scattering (experiments)

ARXIV EPRINT: 2004.10894 


\section{Contents}

1 Introduction 1

2 ATLAS detector 3

3 Data and simulation samples 4

4 Event reconstruction $\quad 6$

5 Event selection $\quad \mathbf{9}$

5.1 Baseline selection $\quad 9$

$\begin{array}{llr}5.2 & \text { Follow-up selection } & 10\end{array}$

6 Signal and background parameterisation $\quad 10$

$\begin{array}{lll}7 & \text { Systematic uncertainties } & 11\end{array}$

8 Results $\quad 14$

$\begin{array}{lll}\text { 8.1 Limits on the visible cross-section } & 15\end{array}$

8.2 Interpretation of the wino-like $\tilde{\chi}_{1}^{ \pm} \tilde{\chi}_{2}^{0} \rightarrow W^{ \pm} \tilde{\chi}_{1}^{0} h \tilde{\chi}_{1}^{0}$ model 16

$\begin{array}{lll}8.3 & \text { Interpretation of the higgsino-like } h \tilde{G} h \tilde{G} \text { model } & 17\end{array}$

$\begin{array}{llr}9 & \text { Conclusion } & 18\end{array}$

$\begin{array}{lr}\text { The ATLAS collaboration } & 29\end{array}$

\section{Introduction}

Theoretical and experimental arguments suggest that the Standard Model (SM) is an effective theory valid up to a certain energy scale. The SM Higgs boson, denoted by $h$, is observed by the ATLAS and CMS collaborations [1-4]. The Higgs boson mass is strongly sensitive to quantum corrections from physics at very high energy scales and demands a high level of fine-tuning, known as the hierarchy problem [5-8]. Supersymmetry (SUSY) [9-14] resolves the hierarchy problem by introducing, for each known particle state, a new partner (superpartner) that shares the same mass and internal quantum numbers with the exception of spin if supersymmetry is unbroken. However, these superpartners have not been observed, so SUSY must be a broken symmetry and the mass scale of the supersymmetric particles is as yet undetermined. The possibility of a supersymmetric dark matter candidate [15, 16] is closely related to the conservation of $R$-parity [17]. Under the $R$-parity conservation hypothesis, the lightest supersymmetric particle (LSP) is stable. If the LSP is weakly interacting, it may provide a viable dark matter candidate. The nature 


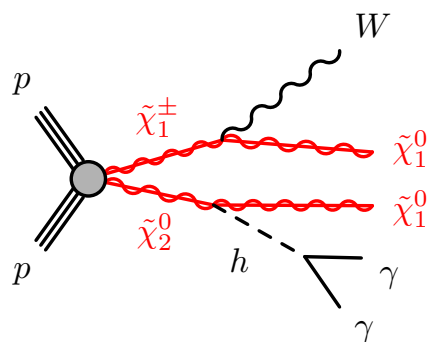

(a)

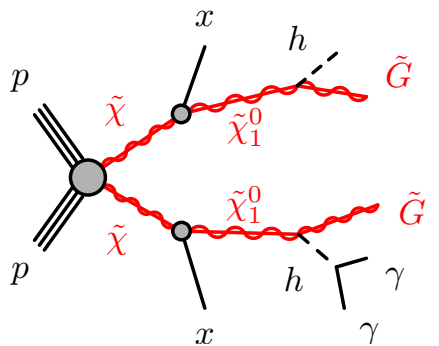

(b)

Figure 1. Signal diagrams illustrating (a) $\tilde{\chi}_{1}^{ \pm} \tilde{\chi}_{2}^{0}$ production, and (b) a higgsino production mode from a GMSB model: $\tilde{\chi}_{1}^{0} \rightarrow h \tilde{G}$. For $\tilde{\chi}_{1}^{ \pm} \tilde{\chi}_{2}^{0}$ production, the lightest chargino $\left(\tilde{\chi}_{1}^{ \pm}\right)$and nextto-lightest neutralino $\left(\tilde{\chi}_{2}^{0}\right)$ are nearly mass degenerate. In the higgsino models, the two lightest neutralinos, $\tilde{\chi}_{1}^{0}$ and $\tilde{\chi}_{2}^{0}$, and the lightest chargino $\tilde{\chi}_{1}^{ \pm}$are approximately mass degenerate, and the $\tilde{\chi}_{1}^{0}$ is the lightest of the four nearly degenerate higgsino states, $x$ is the particle with low momentum from the promptly decay of $\tilde{\chi}_{1}^{ \pm}$and $\tilde{\chi}_{2}^{0}$.

of the LSP is defined by the mechanism that spontaneously breaks supersymmetry and the parameters of the chosen theoretical framework.

In the SUSY scenarios considered as a first benchmark in this paper, the LSP is the lightest of the neutralinos $\tilde{\chi}_{j}^{0}(j=1,2,3,4)$ that, together with the charginos $\tilde{\chi}_{i}^{ \pm}(i=1,2)$, represent the mass eigenstates formed from the mixture of the $\gamma, W, Z$ and Higgs bosons' superpartners (the winos, binos and higgsinos). The neutralinos and charginos are collectively referred to as electroweakinos. Specifically, the electroweakino mass eigenstates are designated in order of increasing mass. Naturalness considerations [18, 19] suggest that the lightest of the charginos and neutralinos have masses near the electroweak scale. Their direct production may be the dominant mechanism at the Large Hadron Collider (LHC) if the superpartners of the gluons and quarks are heavier than a few TeV. In SUSY models where the heaviest (pseudoscalar, charged) minimal supersymmetric Standard Model (MSSM) Higgs bosons and the superpartners of the leptons have masses larger than those of the lightest chargino and next-to-lightest neutralino, the former might decay into the $\tilde{\chi}_{1}^{0}$ and a $W$ boson $\left(\tilde{\chi}_{1}^{ \pm} \rightarrow W \tilde{\chi}_{1}^{0}\right)$, while the latter could decay into the $\tilde{\chi}_{1}^{0}$ and the lightest MSSM Higgs boson or $Z$ boson $\left(\tilde{\chi}_{2}^{0} \rightarrow h / Z \tilde{\chi}_{1}^{0}\right)$ [17, 20, 21]. The decay via the Higgs boson is dominant for many choices of parameters as long as the mass-splitting between the two lightest neutralinos is larger than the Higgs boson mass and the higgsinos are heavier than the winos. SUSY models of this kind could provide a possible explanation for the discrepancy between measurements of the muon's anomalous magnetic moment $g-2$ and SM predictions [22-25].

This paper presents a search in proton-proton $(p p)$ collisions produced at the LHC at a centre-of-mass energy $\sqrt{s}=13 \mathrm{TeV}$ for the direct pair production of electroweakinos that promptly decay into the LSP, producing at least one Higgs boson, decaying into two photons in each event. The primary model, for which the search is optimised, involves the production of a chargino in association with a next-to-lightest neutralino, which promptly decay as $\tilde{\chi}_{1}^{ \pm} \rightarrow W \tilde{\chi}_{1}^{0}$ and $\tilde{\chi}_{2}^{0} \rightarrow h \tilde{\chi}_{1}^{0}$ respectively (see figure 1a), the $\tilde{\chi}_{1}^{0}$ in the final state 
leading to a signature of missing transverse momentum, whose magnitude is denoted by $E_{\mathrm{T}}^{\text {miss }}$. A simplified SUSY model $[26,27]$ is considered for the optimisation of the search and the interpretation of results. The $\tilde{\chi}_{1}^{ \pm} \rightarrow W \tilde{\chi}_{1}^{0}$ and $\tilde{\chi}_{2}^{0} \rightarrow h \tilde{\chi}_{1}^{0}$ decays are each assumed to have a $100 \%$ branching fraction. The Higgs boson branching fractions are assumed to be the same as in the SM [28]. The result from the CMS experiment using an integrated luminosity of $77.5 \mathrm{fb}^{-1}$ of $p p$ collision data is given in ref. [29]. Although the branching fraction of the SM Higgs boson decaying into a pair of photons is small, the diphoton system presented in this paper falls in a narrower mass range around the Higgs boson mass than in refs. [30, 31] where the SM Higgs boson decay into a pair of $b$-quarks. With the diphoton trigger, this channel is more sensitive in the low $E_{\mathrm{T}}^{\text {miss }}$ region than the channel with the SM Higgs boson decaying into a pair of $b$-quarks, which relies on the high $E_{\mathrm{T}}^{\text {miss }}$ trigger. In addition, a prior search from ATLAS [32] for this process making use of $36.1 \mathrm{fb}^{-1}$ of $p p$ collision data, based purely on leptonic decays of the $W$ boson, observed a small excess of events above the SM prediction. This prior search is also updated to the full Run 2 data, and referred to as 'follow-up' analysis.

The analysis optimised for the search for $\tilde{\chi}_{1}^{ \pm} \tilde{\chi}_{2}^{0}$ production is also used to search for a gauge-mediated supersymmetry breaking (GMSB) [33-35] scenario featuring direct production of pairs of higgsinos [36-38], collectively denoted by $\tilde{\chi} \tilde{\chi}$. In this model, the two lightest neutralinos, $\tilde{\chi}_{1}^{0}$ and $\tilde{\chi}_{2}^{0}$, and the lightest chargino $\tilde{\chi}_{1}^{ \pm}$are approximately mass degenerate, and the $\tilde{\chi}_{1}^{0}$ is the lightest of the four nearly degenerate higgsino states. The masses are assumed to be related by $m\left(\tilde{\chi}_{1}^{ \pm}\right)=m\left(\tilde{\chi}_{2}^{0}\right)=m\left(\tilde{\chi}_{1}^{0}\right)+1 \mathrm{GeV}$. The effective cross-section for higgsino production is a combination of the cross-sections for $\tilde{\chi}_{1}^{0} \tilde{\chi}_{2}^{0}, \tilde{\chi}_{1}^{0}$ $\tilde{\chi}_{1}^{ \pm}, \tilde{\chi}_{2}^{0} \tilde{\chi}_{1}^{ \pm}$, and $\tilde{\chi}_{1}^{ \pm} \tilde{\chi}_{1}^{\mp}$ production. In the GMSB scenarios considered (figure 1b), a $100 \%$ branching fraction for $\tilde{\chi}_{1}^{0} \rightarrow h \tilde{G}$ is assumed, where $\tilde{G}$ indicates the gravitino (the superpartner of the graviton). This scenario is denoted by $h \tilde{G} h \tilde{G}$ in the following. In this scenario, $\tilde{G}$ in the final state is stable, weakly interacting, and nearly massless, which leads to an $E_{\mathrm{T}}^{\mathrm{miss}}$ signature.

The general strategy of the analysis is to search for beyond the Standard Model (BSM) events by using a simultaneous signal-plus-background fit to the full $m_{\gamma \gamma}$ spectrum for different categories. The paper is organised as follows. Section 2 presents a brief description of the ATLAS detector. Section 3 introduces the data, the signal and background Monte Carlo (MC) simulation samples used. Section 4 outlines the event reconstruction, while section 5 explains the optimisation of the event selection and categorisation. Section 6 discusses the signal and background modelling. Section 7 summarises the experimental and theoretical systematic uncertainties that affect the results. Section 8 describes the results and their interpretations, and conclusions are drawn in section 9 .

\section{ATLAS detector}

The ATLAS detector [39] is a multipurpose particle detector with a forward-backward symmetric cylindrical geometry and nearly $4 \pi$ coverage in solid angle. ${ }^{1}$ The inner tracking

\footnotetext{
${ }^{1}$ ATLAS uses a right-handed coordinate system with its origin at the nominal interaction point in the centre of the detector. The positive $x$-axis is defined by the direction from the interaction point to the
} 
detector (ID) consists of pixel and microstrip silicon detectors covering the pseudorapidity region $|\eta|<2.5$, surrounded by a transition radiation tracker that enhances electron identification in the region $|\eta|<2.0$. A new inner pixel layer, the insertable B-layer [40, 41], was added at a mean radius of $3.3 \mathrm{~cm}$ during the period between Run 1 and Run 2 of the LHC. The inner detector is surrounded by a thin superconducting solenoid providing an axial $2 \mathrm{~T}$ magnetic field and by a lead/liquid-argon electromagnetic (EM) sampling calorimeter covering $|\eta|<3.2$, with a fine-granularity region up to $|\eta|=2.5$. A steel/scintillatortile hadronic sampling calorimeter provides coverage in the central pseudorapidity range $(|\eta|<1.7)$. The endcap and forward regions $(1.5<|\eta|<4.9)$ of the hadronic calorimeter are made of liquid-argon active layers with either copper or tungsten as the absorber material. A muon spectrometer with an air-core toroid magnet system surrounds the calorimeters. Three layers of high-precision tracking chambers provide coverage in the range $|\eta|<2.7$, while dedicated fast chambers allow triggering in the region $|\eta|<2.4$. The ATLAS trigger system consists of a hardware-based first-level trigger followed by a software-based high-level trigger [42].

\section{Data and simulation samples}

The analysis uses $p p$ collision data with a bunch crossing interval of $25 \mathrm{~ns}$, collected from 2015 to 2018 at $\sqrt{s}=13 \mathrm{TeV}$. Only events that were recorded in stable beam conditions, when relevant detector components were functioning properly, are considered. A diphoton trigger [43] was used to collect the events by requiring two reconstructed photon candidates with transverse energies $\left(E_{\mathrm{T}}\right)$ of at least $35 \mathrm{GeV}$ and $25 \mathrm{GeV}$ for the $E_{\mathrm{T}^{-}}$ordered leading and subleading photons respectively. The trigger efficiency relative to the offline-reconstructed photons was $99 \%$. The data sample corresponds to an integrated luminosity of $139.0 \pm$ $2.4 \mathrm{fb}^{-1}$. There are, on average, 25 to 38 interactions in the same bunch crossing (in-time pile-up) in the data sample.

The MC simulation of signal and background processes is used to optimise the selection criteria, estimate uncertainties and study the shapes of the signal and background diphoton invariant mass $\left(m_{\gamma \gamma}\right)$ distributions. Signal events were generated with up to two additional partons in the matrix element using MADGRAPH_aMC@NLO 2.6.2 [44] at leading order (LO) in quantum chromodynamics (QCD) using the NNPDF3.0LO [45] parton distribution function (PDF) set and CKKW-L merging scheme. Parton showering and hadronisation were handled by the PyтhiA 8.230 [46] event generator with the A14 [47] set of tuned parameters (tune), using the NNPDF2.3LO PDF set [48]. MC samples for the $\tilde{\chi}_{1}^{ \pm} \tilde{\chi}_{2}^{0}$ production were generated assuming $m\left(\tilde{\chi}_{1}^{ \pm}\right)=m\left(\tilde{\chi}_{2}^{0}\right)$ for a range of values of $m\left(\tilde{\chi}_{1}^{0}\right)$. As shown in figure $2 \mathrm{a}$, the transverse momentum $\left(p_{\mathrm{T}}\right)$ distribution of the $\tilde{\chi}_{1}^{0} \tilde{\chi}_{1}^{0}$ system is broader for higher values of the difference $m\left(\tilde{\chi}_{1}^{ \pm} / \tilde{\chi}_{2}^{0}\right)-m\left(\tilde{\chi}_{1}^{0}\right)$. The $p_{\mathrm{T}}$ distributions of

centre of the LHC ring, with the positive $y$-axis pointing upwards, while the beam direction defines the $z$-axis. Cylindrical coordinates $(r, \phi)$ are used in the transverse plane, $\phi$ being the azimuthal angle around the $z$-axis. The pseudorapidity $\eta$ is defined in terms of the polar angle $\theta$ by $\eta=-\ln \tan (\theta / 2)$. Rapidity is defined as $y=0.5 \ln \left[\left(E+p_{z}\right) /\left(E-p_{z}\right)\right]$ where $E$ denotes the energy and $p_{z}$ is the component of the momentum along the beam direction. The angular distance $\Delta R$ is defined as $\sqrt{(\Delta y)^{2}+(\Delta \phi)^{2}}$. 


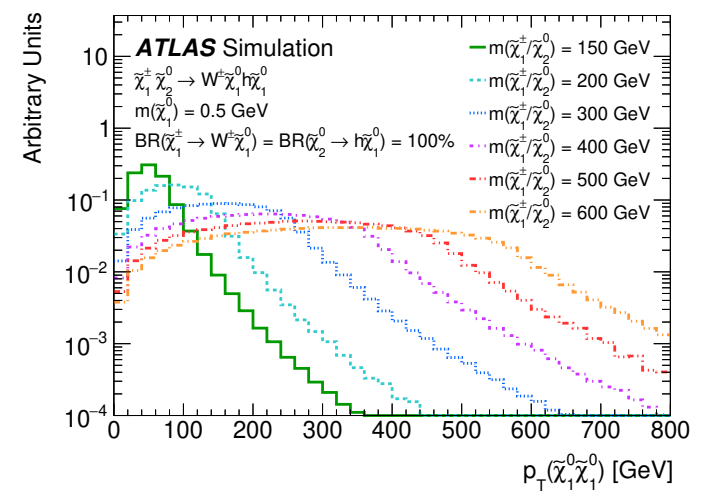

(a)

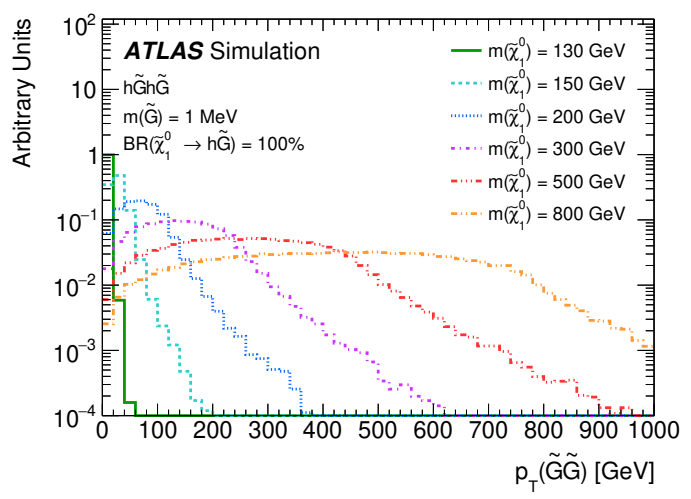

(b)

Figure 2. The $p_{\mathrm{T}}$ distribution of (a) the $\tilde{\chi}_{1}^{0} \tilde{\chi}_{1}^{0}$ in $W^{ \pm} \tilde{\chi}_{1}^{0} h \tilde{\chi}_{1}^{0}$ production and (b) $\tilde{G} \tilde{G}$ in $h \tilde{G} h \tilde{G}$ production.

the $\tilde{G} \tilde{G}$ system for the higgsino production of $h \tilde{G} h \tilde{G}$ are presented in figure $2 \mathrm{~b}$. The MC samples include $\tilde{\chi}_{1}^{0} \tilde{\chi}_{2}^{0}, \tilde{\chi}_{1}^{0} \tilde{\chi}_{1}^{ \pm}, \tilde{\chi}_{2}^{0} \tilde{\chi}_{1}^{ \pm}$, and $\tilde{\chi}_{1}^{ \pm} \tilde{\chi}_{1}^{\mp}$ production. The kinematic distributions depend strongly on the mass of the $\tilde{\chi}_{1}^{0}$, where the mass of the $\tilde{G}$ is assumed to be $1 \mathrm{MeV}$.

Signal cross-sections were calculated to NLO in the strong coupling constant, $\alpha_{\mathrm{S}}$, adding the resummation of soft gluon emission at next-to-leading-logarithm accuracy $(\mathrm{NLO}+\mathrm{NLL})$ [49-53]. The nominal cross-section and its uncertainty are taken from an envelope of cross-section predictions using different PDF sets and factorisation and renormalisation scales, as described in ref. [54].

The dominant backgrounds are resonant SM $h \rightarrow \gamma \gamma$ processes, and non-resonant processes that include $\gamma \gamma, \gamma+$ jets, $V \gamma(V=W, Z)$ and $V \gamma \gamma$ production. Both the shape and normalisation of the total non-resonant background are obtained directly from data, as described in section 6 . Simulation events for the total non-resonant background are used in figure 3 and for the choice of background analytic parametrisation as described in section 6 . For the production of the resonant SM Higgs boson, events from the $W h$ and $Z h$ processes were generated with PYTHIA 8.186 with the A14 tune and the NNPDF2.3LO PDF set. The gluon-gluon fusion ( $\mathrm{ggF}$ ) and vector-boson fusion (VBF) samples were generated with Powheg-Box v2 [55-59] interfaced to Pythia 8.186 with the AZNLO [60] tune and the CT10 PDF set [61]. Samples of $t \bar{t} h$ events were generated with MAdGraPH_aMC@NLO 2.2.3 interfaced to Pythia 8.186 with the NNPDF3.0LO PDF set. Samples of $b \bar{b} h$ events were generated with MADGRAPH_aMC@NLO 2.2.3 interfaced to PyTHIA 8.186 with the A14 tune and the NNPDF2.3LO PDF set. The non-resonant diphoton processes with associated jets were generated using SHERPA 2.2.4 [62]. Matrix elements (ME) were calculated with up to three partons at LO and merged with the SHERPA 2.2.4 parton shower (PS) [63] using the ME+PS@LO prescription [64]. The CT10 PDF set was used in conjunction with a dedicated parton-shower tuning developed by the authors of SHERPA 2.2.4. The $V \gamma$ and $V \gamma \gamma$ samples were generated using SHERPA 2.2.4 with the CT10 PDF set.

The cross-sections for the SM Higgs boson processes were calculated at next-to-leading order (NLO) in electroweak theory and next-to-next-to-leading order (NNLO) in QCD for the VBF, $Z h$ and $W h$ samples $[28,65-71]$ and next-to-next-to-next-to-leading order plus 


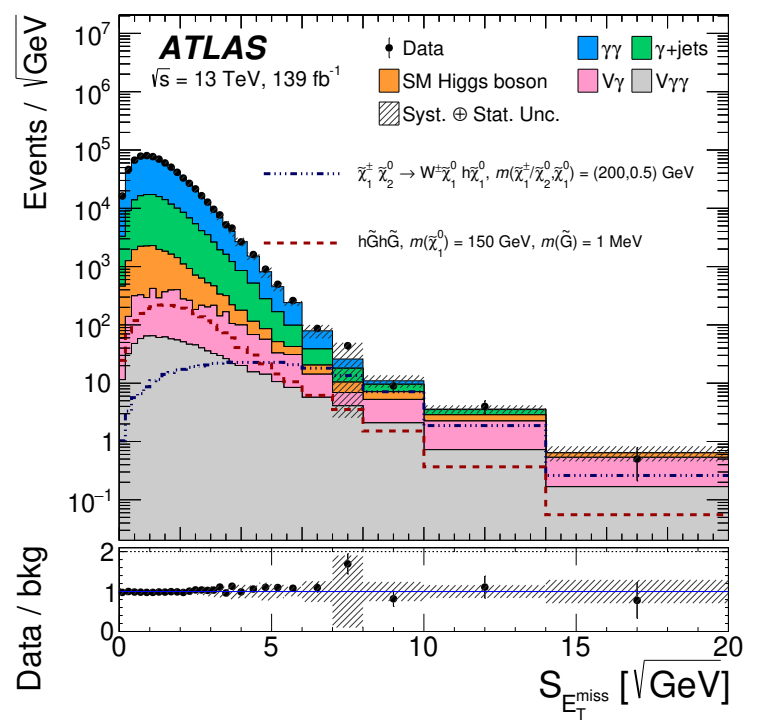

Figure 3. The distribution of $S_{E_{\mathrm{T}}^{\text {miss }}}$ after the selection of diphoton candidates with $120<m_{\gamma \gamma}<$ $130 \mathrm{GeV}$. Expected distributions are shown for the $\tilde{\chi}_{1}^{ \pm} \tilde{\chi}_{2}^{0} \rightarrow W^{ \pm} \tilde{\chi}_{1}^{0} h \tilde{\chi}_{1}^{0}$ signal with $m\left(\tilde{\chi}_{1}^{ \pm} / \tilde{\chi}_{2}^{0}\right)=$ $200 \mathrm{GeV}$ and $m\left(\tilde{\chi}_{1}^{0}\right)=0.5 \mathrm{GeV}$, and the $h \tilde{G} h \tilde{G}$ signal with $m\left(\tilde{\chi}_{1}^{0}\right)=150 \mathrm{GeV}$ and $m(\tilde{G})=1 \mathrm{MeV}$. These overlaid signal points are representative of the model kinematics. The sum in quadrature of the MC statistical and experimental systematic uncertainties in the total background is shown as the hatched bands, while the theoretical uncertainties in the background normalisation are not included. The $t t \gamma$ and $t t \gamma \gamma$ processes have a negligible contribution and are not represented. Overflow events are included in the rightmost bin. The lower panel shows the ratio of the data to the background, called "bkg".

next-to-next-to-leading logarithm $\left(\mathrm{N}^{3} \mathrm{LO}+\mathrm{NNLL}\right)$ in QCD for the ggF sample [28, 72-75]. The $t \bar{t} h$ cross-section was calculated with NLO accuracy in QCD with NLO electroweak corrections [76-79]. The $b \bar{b} h$ cross-section was obtained by matching the five-flavor scheme cross section accurate to NNLO in QCD with the four-flavor scheme cross section accurate to NLO in QCD [80-82]. The SM Higgs boson mass was set to $125.09 \mathrm{GeV}$ [3] and its branching fraction to decay into two photons was $0.227 \%$ [28].

Different pile-up conditions from same and neighbouring bunch crossings as a function of the instantaneous luminosity were simulated by overlaying minimum-bias events, generated with Pythia 8.186 with the MSTW2008LO PDF set [83] and the A3 [84] tune, onto all hard-process events. Differences between the simulated and observed distributions of the number of interactions per bunch crossing were corrected for by applying weights to simulated events. Detector effects were simulated using a full simulation [85] performed using GEANT4 [86] for the signals, SM Higgs boson processes, $V \gamma$ and $V \gamma \gamma$ backgrounds. The diphoton continuum background and some of the signal samples were simulated using a fast simulation of the calorimeter based on AtLFASTII [85].

\section{Event reconstruction}

Photons are reconstructed in the region $|\eta|<2.37$, excluding the EM calorimeter transition region $1.37<|\eta|<1.52$, from clusters of energy deposits in the EM calorimeters. Clusters 
without a matching track or reconstructed conversion vertex in the ID are classified as unconverted photons. Those with a matching reconstructed conversion vertex or with a matching track, consistent with originating from a photon conversion, are classified as converted photons. The reconstruction efficiency is $99 \%$ for photons and the conversion reconstruction efficiency is $70 \%$ [87]. The photon energy is calibrated using a multivariate regression algorithm trained with fully reconstructed $\mathrm{MC}$ samples and then corrected using data-driven techniques [87]. The overall energy scale in data and the difference in the constant term on the resolution between data and simulation are estimated from using a sample with $Z$ boson decays into electrons [87]. The photon direction is estimated using either EM calorimeter longitudinal segmentation (if unconverted) or the conversion vertex position (if converted), together with constraints from the $p p$ collision point.

To reduce the misidentification of hadronic jets containing a high- $p_{\mathrm{T}}$ neutral hadron (e.g. $\pi^{0}$ ) decaying into two photons, 'Tight' identification criteria [87] are applied. The photon identification is based on the lateral profile of the energy deposits in the first and second layers of the EM calorimeter, and on the shower leakage fraction in the hadronic calorimeter. The selection requirements are tuned for converted and unconverted photon candidates, separately. The identification efficiency for unconverted and converted photons ranges from $85 \%$ to $99 \%$ between $30 \mathrm{GeV}$ and $250 \mathrm{GeV}$ [87]. Corrections are applied to the EM shower-shape variables for simulated photons, to account for small differences between data and simulation.

To further suppress hadronic backgrounds, requirements on two photon isolation variables are applied. The first variable, $E_{\mathrm{T}}^{\text {iso }}$, calculates the sum of the transverse energies deposited in topological clusters [88] in the calorimeter within a cone of size $\Delta R=0.2$ around each photon. The photon cluster energy and an estimate of the energy deposited by the photon outside its associated cluster are also subtracted from this sum. To reduce underlying-event and pile-up effects, $E_{\mathrm{T}}^{\text {iso }}$ is further corrected using the method described in refs. [89-91]. The second variable expresses track-based isolation, defined as the scalar sum of the transverse momenta of all tracks with $p_{\mathrm{T}}>1 \mathrm{GeV}$ and consistent with originating from the primary vertex (PV) within a cone of size $\Delta R=0.2$ around each photon. The isolation efficiency for photons, which is mostly independent of their kinematic variables, is about $90 \%$.

Events are required to have at least one PV, defined as a vertex associated with at least two tracks with $p_{\mathrm{T}}>0.5 \mathrm{GeV}$. In each event, the PV most likely to be the origin of the diphoton, selected from the PV candidates using a neural network [92], is required to be consistent with the PV with the highest sum of squared transverse momenta of associated tracks. The neural network algorithm selects a diphoton vertex within $0.3 \mathrm{~mm}$ of the true $h \rightarrow \gamma \gamma$ production vertex in $79 \%$ of simulated gluon-gluon fusion events. For the other Higgs production modes this fraction ranges from $84 \%$ to $97 \%$, increasing with jet activity or the presence of charged leptons [92].

Electrons are reconstructed from energy deposits measured in the EM calorimeter that are matched to tracks from ID [87]. They are required to satisfy $|\eta|<2.47$, excluding the EM calorimeter transition region $1.37<|\eta|<1.52$, and to have $p_{\mathrm{T}}>10 \mathrm{GeV}$. The electrons are identified using a likelihood-based algorithm that uses track and shower-shape variables. 
The 'MediumLLH' criteria are applied, providing an identification efficiency varying from $85 \%$ to $95 \%$ as a function of $E_{\mathrm{T}}$ [87]. Loose calorimeter and track isolation requirements are applied to electrons. The efficiency of the isolation requirements is $98 \%$ [93].

Muons are reconstructed from high-quality track segments in the muon spectrometer. In the region $|\eta|<2.5$, they must be matched to ID tracks. They are required to have $p_{\mathrm{T}}>10 \mathrm{GeV}$ and $|\eta|<2.7$. The muon 'medium' criteria are applied with a 96\% [94] identification efficiency. The muon candidates must also satisfy loose calorimeter and track isolation criteria. The combined isolation efficiency varies from $95 \%$ to $99 \%$ as a function of $p_{\mathrm{T}}$ from $25 \mathrm{GeV}$ to $60 \mathrm{GeV}$ [94].

The significance of the track's transverse impact parameter relative to the PV is required to be $\left|d_{0}\right| / \sigma_{d_{0}}<5$ (3) for electrons (muons). The longitudinal impact parameter $z_{0}$ must satisfy $\left|z_{0}\right| \sin \theta<0.5 \mathrm{~mm}$ for electrons and muons.

Jets are reconstructed from three-dimensional topological clusters using the anti- $k_{t}$ algorithm $[95,96]$ with a radius parameter of $R=0.4$. The jets are required to have $p_{\mathrm{T}}>20 \mathrm{GeV}$ and $|\eta|<4.5$ for the $E_{\mathrm{T}}^{\text {miss }}$ calculation and $p_{\mathrm{T}}>25 \mathrm{GeV}$ and $|\eta|<4.4$ for the event selection. Jets with $|\eta|<2.4$ and $p_{\mathrm{T}}<60 \mathrm{GeV}$ must satisfy the jet vertex tagger (JVT) selection [97], in which a jet is identified as originating from the PV depending on a likelihood value calculated from the track information. In addition, quality criteria are applied to the jets, and events with jets consistent with noise in the calorimeter or non-collision backgrounds are rejected [98].

Reconstruction ambiguities between photons, electrons, muons, and jets are resolved using an 'overlap removal' procedure among all the objects in the following order. First, electrons, muons, and jets found within $\Delta R=0.4$ of a photon are removed. Next, jets found within $\Delta R=0.2$ of an electron are removed. Lastly, electrons and muons within $\Delta R=0.4$ of the remaining jets are removed. A different overlap removal strategy was used in the previous study [32] and the selection is discussed in section 5.2. It was motivated by the prioritisation of electrons, as opposed to photons. The results show no significant difference in sensitivity between these two strategies.

Jets containing a $b$-hadron are identified using the MV2c10 $[99,100]$ multivariate discriminant built with information from track impact parameters and the presence of reconstructed secondary vertices, which applies a multi-vertex fitter to reconstruct the hadron decay chain $b \rightarrow c$. A value of the discriminating variable is chosen such that it provides a $b$-tagging efficiency of $70 \%$ in simulated $t \bar{t}$ events. The rejection for $c$-jets and jets originating from gluons or light $(u, d, s)$ quarks are 8.9 and 300 [99], respectively. An additional energy correction is applied to $b$-jets to account for the presence of muons in the jet [99].

The $E_{\mathrm{T}}^{\text {miss }}$ is calculated as the magnitude of the negative vectorial sum of the transverse momenta of calibrated photons, electrons, muons and jets associated with the PV. The transverse momenta of all remaining tracks that originate from the PV but are not already used in the $E_{\mathrm{T}}^{\text {miss }}$ calculation are summed and taken into account in the $E_{\mathrm{T}}^{\text {miss }}$ calculation. This term is defined as the track-based soft term [101]. In this way, the $E_{\mathrm{T}}^{\text {miss }}$ is adjusted for the best calibration of the jets and the other identified physics objects above, while maintaining pileup independence in the soft term. 


\section{Event selection}

\subsection{Baseline selection}

Each event is first required to contain at least two photons with $p_{\mathrm{T}}>22 \mathrm{GeV}$. The photons are ordered by their $p_{\mathrm{T}}$. The leading and subleading photons are then required to have $p_{\mathrm{T}}^{\gamma} / m_{\gamma \gamma}>0.35$ and 0.25 , respectively, where $m_{\gamma \gamma}$ is the invariant mass of the leading and subleading photon pair. The signal region is defined as $105<m_{\gamma \gamma}<160 \mathrm{GeV}$, where $m_{\gamma \gamma}$ is calculated using the photon momentum vectors recomputed relative to the PV. The selected events are divided into 12 categories based on the number of leptons $\left(N_{\ell}\right)$, number of jets $\left(N_{j}\right)$, the invariant mass of the two highest- $p_{\mathrm{T}}$ jets $\left(m_{j j}\right)$, and the $E_{\mathrm{T}}^{\mathrm{miss}}$ significance $S_{E_{\mathrm{T}}^{\text {miss }}}=E_{\mathrm{T}}^{\text {miss }} / \sqrt{\sum E_{\mathrm{T}}}$. The total transverse energy $\sum E_{\mathrm{T}}$ is calculated from the scalar sum of the transverse momenta of the calibrated photons, electrons, muons and jets used in the $E_{\mathrm{T}}^{\text {miss }}$ calculation described in section 4 , as well as the tracks not associated with these but consistent with originating from the PV. Because both the $E_{\mathrm{T}}^{\text {miss }}$ and $\sum E_{\mathrm{T}}$ resolutions increase linearly with the number of pileup events, $S_{E_{\mathrm{T}}^{\mathrm{miss}}}$ is more resilient to pileup than $E_{\mathrm{T}}^{\mathrm{miss}}$. No $b$-jet veto is applied in the baseline selection. The 12 categories are defined in table 1 . The $\tilde{\chi}_{1}^{ \pm} \tilde{\chi}_{2}^{0}$ signal sample with $m\left(\tilde{\chi}_{1}^{ \pm} / \tilde{\chi}_{2}^{0}\right)=150 \mathrm{GeV}$ and $m\left(\tilde{\chi}_{1}^{0}\right)=0.5 \mathrm{GeV}$ is used to optimise the boundary of each category to maximise the significance when combining all 12 categories. This signal point has low $E_{\mathrm{T}}^{\text {miss }}$, where the diphoton channel is expected to have a better sensitivity than the channel with the SM Higgs boson decaying into a pair of $b$-quarks [30, 31]. The 'Leptonic' and 'Hadronic' categories are used to accommodate the most clearly identifiable leptonic and hadronic decays of the $W$ boson, while the 'Rest' category retains all additional signal topologies. The signal $\tilde{\chi}_{1}^{ \pm} \tilde{\chi}_{2}^{0} \rightarrow W^{ \pm} \tilde{\chi}_{1}^{0} h \tilde{\chi}_{1}^{0}$ has the highest expected significance in the Leptonic categories, and the $h \tilde{G} h \tilde{G}$ signals have the highest expected significance in the Rest categories. Because the different signal models and mass points have different $p_{\mathrm{T}}$ distributions as shown in figure 2 , and since $p_{\mathrm{T}}$ and $S_{E_{\mathrm{T}}^{\mathrm{miss}}}$ distributions are highly correlated, each region is divided into $S_{E_{\mathrm{T}}^{\mathrm{miss}}}$ bins to improve the sensitivity. The regions do not change significantly if a different mass point is used for optimisation.

Figure 3 shows the distribution of $S_{E_{T}^{\text {miss }}}$ after the selection of diphoton candidates with $120<m_{\gamma \gamma}<130 \mathrm{GeV}$, where signal dominates. The shapes and normalisations of the $V \gamma$ and $V \gamma \gamma$ contributions are obtained from the MC simulation. The shape of the $\gamma \gamma$ contribution is obtained from the $\mathrm{MC}$ simulation while the normalisation is fixed to the yields in the sidebands $\left(105<m_{\gamma \gamma} \leq 120 \mathrm{GeV}, 130 \leq m_{\gamma \gamma}<160 \mathrm{GeV}\right)$ of the data multiplied by the diphoton purity among all the backgrounds. The diphoton purity is measured in the data, using a two-dimensional sideband technique by counting the number of events in which one or both photons satisfy or fail to satisfy the identification or isolation requirements [102]. The diphoton purity varies from $65 \%$ to $93 \%$ for different categories. The shape of the $\gamma+$ jets contribution is obtained using the data distribution in a control region where the event selection is the same as for the signal region but one of the photons fails to satisfy the identification criteria, after subtracting the contamination from $\gamma \gamma, V \gamma$ and $V \gamma \gamma$ using MC simulation. Its normalisation is fixed to the $\gamma+$ jets purity and varies from $34 \%$ to $7 \%$ of the total yield in different categories. 


\begin{tabular}{|ccc|}
\hline Channels & Names & Selection \\
\hline \multirow{5}{*}{ Leptonic } & Category 1 & $0<S_{E_{\mathrm{T}}^{\text {miss }}} \leq 2, N_{\ell} \geq 1$ \\
& Category 2 & $2<S_{E_{\mathrm{T}}^{\text {miss }}} \leq 4, N_{\ell} \geq 1$ \\
& Category 3 & $4<S_{E_{\mathrm{T}}^{\text {miss }}} \leq 6, N_{\ell} \geq 1$ \\
& Category 4 & $S_{E_{\mathrm{T}}^{\text {miss }}}>6, N_{\ell} \geq 1$ \\
\hline \multirow{5}{*}{ Hadronic } & Category 5 & $5<S_{E_{\mathrm{T}}^{\text {miss }}} \leq 6, N_{\ell}=0, N_{j} \geq 2, m_{j j} \in[40,120] \mathrm{GeV}$ \\
& Category 6 & $6<S_{E_{\mathrm{T}}^{\text {miss }}} \leq 7, N_{\ell}=0, N_{j} \geq 2, m_{j j} \in[40,120] \mathrm{GeV}$ \\
& Category 7 & $7<S_{E_{\mathrm{T}}^{\text {miss }}} \leq 8, N_{\ell}=0, N_{j} \geq 2, m_{j j} \in[40,120] \mathrm{GeV}$ \\
& Category 8 & $S_{E_{\mathrm{T}}^{\text {miss }}}>8, N_{\ell}=0, N_{j} \geq 2, m_{j j} \in[40,120] \mathrm{GeV}$ \\
\hline \multirow{5}{*}{ Rest } & Category 9 & $6<S_{E_{\mathrm{T}}^{\text {miss }}} \leq 7, N_{\ell}=0, N_{j}<2$ or $\left(N_{j} \geq 2, m_{j j} \notin[40,120] \mathrm{GeV}\right)$ \\
& Category 10 & $7<S_{E_{\mathrm{T}}^{\text {miss }}} \leq 8, N_{\ell}=0, N_{j}<2$ or $\left(N_{j} \geq 2, m_{j j} \notin[40,120] \mathrm{GeV}\right)$ \\
& Category 11 & $8<S_{E_{\mathrm{T}}^{\text {miss }}} \leq 9, N_{\ell}=0, N_{j}<2$ or $\left(N_{j} \geq 2, m_{j j} \notin[40,120] \mathrm{GeV}\right)$ \\
& Category 12 & $S_{E_{\mathrm{T}}^{\text {miss }}}>9, N_{\ell}=0, N_{j}<2$ or $\left(N_{j} \geq 2, m_{j j} \notin[40,120] \mathrm{GeV}\right)$ \\
\hline
\end{tabular}

Table 1. Criteria used in the categorisation.

\subsection{Follow-up selection}

To check the small excess of events observed in the previous search from ATLAS using $36.1 \mathrm{fb}^{-1}$ of $p p$ collision data [32], two signal regions ('SR1L $\gamma \gamma-\mathrm{a}$ ' and 'SR1L $\gamma \gamma-\mathrm{b}$ ') defined in the previous search are reused in this analysis. Events are required to have exactly one lepton with $p_{\mathrm{T}}>25 \mathrm{GeV}$ and exactly two photons with $p_{\mathrm{T}}>40(30) \mathrm{GeV}$ for the leading (subleading) photon. The invariant mass of the two photons is required to be $105<m_{\gamma \gamma}<160 \mathrm{GeV}$, with $E_{\mathrm{T}}^{\text {miss }}>40 \mathrm{GeV}$. The difference in azimuthal angle in the transverse plane between the diphoton system and the lepton plus $E_{\mathrm{T}}^{\text {miss }}$ vector is required to be greater than 2.25 radians. To reduce contributions from $t \bar{t} h$, a $b$-jet veto is used in both the signal regions.

To further reduce contributions from SM backgrounds, the transverse mass $m_{\mathrm{T}}^{W}[32]$ of the lepton and $E_{\mathrm{T}}^{\mathrm{miss}}$, and the three-body transverse mass $m_{\mathrm{T}}^{W \gamma i}[32]$ of the lepton, $E_{\mathrm{T}}^{\text {miss }}$ and the $i^{t h}$ photon ordered by $p_{\mathrm{T}}$ are used to define the two orthogonal signal regions. For both signal regions, events are required to have $m_{\mathrm{T}}^{W \gamma 1}>150 \mathrm{GeV}$ and $m_{\mathrm{T}}^{W \gamma 2}>80 \mathrm{GeV}$. The first signal region, 'SR1L $\gamma \gamma$-a', selects events with $m_{\mathrm{T}}^{W}>110 \mathrm{GeV}$ and $m_{\mathrm{T}}^{W \gamma 2}>140 \mathrm{GeV}$ while the events that fail to satisfy these requirements define the second signal region ('SR1L $\gamma \gamma-b$ ').

\section{Signal and background parameterisation}

The signals and the SM Higgs boson background mass distributions are described independently using double-sided Crystal Ball functions (as defined in ref. [103]). The parameter values for the functions are extracted by fitting the diphoton invariant mass distributions of the MC simulation for each category. The expected normalisations are calculated from 
the theoretical cross-sections multiplied by the acceptance and efficiency from the MC simulation.

The normalisation and shape of the non-resonant background are extracted by fitting the diphoton invariant mass distribution in data for each category. Following the method used in the measurement of the SM Higgs boson decaying into two photons [104], several candidate analytic functions are chosen for the non-resonant background parameterisation: the exponential functions of different-order polynomials, Bernstein polynomials of different order, and an adapted dijet function [105]. The potential bias, denoted by $\Delta N_{\mathrm{bkg}}^{\text {non-res }}$, from the functional form modelling the continuum background in each category is estimated. It is defined as the maximal signal yield extracted from the fit to a continuum-background-only diphoton invariant mass distribution. This distribution is taken from MC simulations and is normalised to the integrated luminosity of $139 \mathrm{fb}^{-1}$, with small statistical uncertainty, using a signal-plus-background model. The Higgs boson mass varies from $115 \mathrm{GeV}$ to $135 \mathrm{GeV}$ [104]. This is to ensure the bias from choosing different background models is conservatively estimated. For categories 2 to 12 , the functional form with $\Delta N_{\mathrm{bkg}}^{\text {non-res }}$ less than $20 \%$ of the statistical uncertainty in data and with the fewest free parameters is chosen as the nominal background function. In the case of Category 1, with large MC statistical uncertainty, none of the functional forms satisfies the criterion on the fraction of the statistical uncertainty in data, thus the functional form with the smallest $\Delta N_{\mathrm{bkg}}^{\text {non-res }}$ is chosen. The $\Delta N_{\mathrm{bkg}}^{\text {non-res }}$ value of the chosen functional form is taken as the non-resonant background modelling uncertainty in each category and is shown in table 2 .

\section{$7 \quad$ Systematic uncertainties}

Uncertainties from experimental and theoretical sources that affect the signal efficiency and the SM Higgs boson background yield are estimated from the MC simultation. The non-resonant background is obtained directly from the fit to the data. The only systematic uncertainty in the non-resonant background is the potential bias in $\Delta N_{\mathrm{bkg}}^{\text {non-res }}$ from the choice of background modelling. A summary of the experimental and theoretical uncertainties in the yield from the SM Higgs boson background processes, non-resonant background, and signal production is shown in table 3 .

The uncertainty in the combined 2015-2018 integrated luminosity is $1.7 \%$ [106], obtained using the LUCID-2 detector [107] for the primary luminosity measurements.

The efficiency of the diphoton trigger used to select events is evaluated in MC simulation using a trigger matching technique and in data using a bootstrap method [43]. The uncertainty in the trigger efficiency for events with $105<m_{\gamma \gamma}<160 \mathrm{GeV}$ is found to be $0.4 \%$.

The uncertainty in the vertex selection efficiency is assessed by comparing the efficiency of finding photon-pointing vertices in $Z \rightarrow e^{+} e^{-}$events in data with that in MC simulation [108]. The resulting uncertainty is found to be negligible in the inclusive photon selection.

The systematic uncertainties due to the photon energy scale and resolution are obtained from ref. [87]. The uncertainty in the energy scale has an effect below $1 \%$ on the 


\begin{tabular}{|llcc|}
\hline Category & Function & $\Delta N_{\text {bkg }}^{\text {non-res }}$ & $\Delta N_{\text {bkg }}^{\text {non-res }} / N_{\text {bkg }}^{\text {non-res. }}[\%]$ \\
\hline 1 & $\left(1-x^{1 / 3}\right)^{b} \cdot x^{a}$ & 5.5 & 2.4 \\
2 & $\sum_{j=0}^{3} C_{3}^{j} x^{j}(1-x)^{3-j} b_{j, 3}$ & 1.8 & 2.4 \\
3 & $\exp (a \cdot x)$ & 0.6 & 3.6 \\
4 & $\exp (a \cdot x)$ & 0.3 & 3.7 \\
5 & $\exp (a \cdot x)$ & 1.6 & 2.8 \\
6 & $\exp (a \cdot x)$ & 0.5 & 3.3 \\
7 & $\exp (a \cdot x)$ & 0.3 & 5.1 \\
8 & $\exp (a \cdot x)$ & 0.2 & 4.6 \\
9 & $\exp (a \cdot x)$ & 1.5 & 2.3 \\
10 & $\exp (a \cdot x)$ & 0.6 & 2.5 \\
11 & $\exp (a \cdot x)$ & 0.4 & 5.6 \\
12 & $\exp (a \cdot x)$ & 0.4 & 3.0 \\
\hline
\end{tabular}

Table 2. The analytic functions used to model the non-resonant background, the extracted signals from the background-only fits $\left(\Delta N_{\mathrm{bkg}}^{\text {non-res }}\right)$ to the $\mathrm{MC}$ and the relative uncertainty in the nonresonant background within $120<m_{\gamma \gamma}<130 \mathrm{GeV}\left(\Delta N_{\text {bkg }}^{\text {non-res }} / N_{\text {bkg }}^{\text {non-res. }}\right)$ for each category. The variable $x$ is defined as $m_{\gamma \gamma} / \sqrt{s}$ while $a$ and $b$ are parameters of the background functions. The $C_{3}^{j}$ are binomial coefficients and the $b_{j, 3}$ are the fitted parameters for the third order Bernstein polynomial parameterization.

normalisation of the signals and the SM Higgs boson background in the $p_{\mathrm{T}}$ range of the photons used in the analysis. The uncertainty in the energy resolution has an effect below $2 \%$ on the normalisation of the signals and the SM Higgs boson background. The uncertainties affecting the signal and the SM Higgs boson background mass distributions due to the photon energy scale and resolution are also evaluated. The uncertainties vary from below $1 \%$ to $20 \%$ for different categories and for different SM Higgs boson production processes. Overall, they amount to less than $3 \%$ of the total SM Higgs boson background.

Uncertainties in photon identification and isolation efficiencies are estimated [87], and their impact on the number of events in each category is quantified. The photon identification uncertainty varies in the range $1 \%-3 \%$ for the SM Higgs boson background and $1 \%-2 \%$ for the signals in all categories. The uncertainty in the photon calorimeter isolation efficiency is calculated from efficiency differences between applying and not applying corrections derived from inclusive photon events to the isolation variables in simulation. The measurements of the efficiency correction factors using inclusive photon events are used to derive the uncertainty in the photon track isolation efficiency. The photon isolation efficiency uncertainty is found to be in the range 1\%-3\% for the SM Higgs boson background and $1 \%-2 \%$ for the signals.

Migration of events among categories occurs if the energies of identified particles, jets and the $E_{\mathrm{T}}^{\mathrm{miss}}$, are varied within their uncertainties. The uncertainties in the jet energy scale, resolution [109] and jet vertex tagger are propagated to the $E_{\mathrm{T}}^{\text {miss }}$ calculation. In 


\begin{tabular}{|c|c|c|c|}
\hline \multirow[b]{2}{*}{ Source } & \multirow[b]{2}{*}{ Signals $[\%]$} & \multicolumn{2}{|c|}{ Backgrounds [\%] } \\
\hline & & SM Higgs boson & $\begin{array}{c}\text { Non-resonant } \\
\text { background }\end{array}$ \\
\hline \multicolumn{4}{|l|}{ Experimental } \\
\hline Luminosity & \multicolumn{2}{|r|}{1.7} & - \\
\hline Jets (scale, resolution, JVT) & $0.2-3.3$ & $0.9-31$ & - \\
\hline Electron/Photon (scale, resolution) & $0.3-1.5$ & $0.6-2.7$ & - \\
\hline Photon (identification, isolation, trigger) & $2.2-2.6$ & $2.8-4.3$ & - \\
\hline Electron (identification isolation) & $0.0-0.5$ & $0.0-0.6$ & - \\
\hline Muon (identification, isolation, scale, resolution) & $<0.6$ & $<0.3$ & - \\
\hline$E_{\mathrm{T}}^{\text {miss }}$ reconstruction (jets, soft term) & $<0.7$ & $0.4-14$ & - \\
\hline Pile-up reweighting & $0.3-1.8$ & $1.3-1.5$ & - \\
\hline Non-resonant background modelling & & - & $2-6$ \\
\hline \multicolumn{4}{|l|}{ Theoretical } \\
\hline Factorisation and renormalisation scale & $<1$ & $4.1-6.5$ & - \\
\hline $\mathrm{PDF}+\alpha_{\mathrm{S}}$ & $<6.6$ & $3.3-6.4$ & - \\
\hline Multiple parton-parton interactions & \multicolumn{2}{|r|}{$<1$} & - \\
\hline $\mathcal{B}(H \rightarrow \gamma \gamma)$ & \multicolumn{2}{|r|}{1.73} & - \\
\hline
\end{tabular}

Table 3. Breakdown of the dominant systematic uncertainties. The uncertainties (in \%) in the yield of signals, the background from the SM Higgs boson processes and non-resonant background are shown. All production modes of the SM Higgs boson are considered together. A "- " indicates that the systematic uncertainty is not applicable to the corresponding sample. If a given source has a different impact on the various categories, the given range corresponds to the smallest and largest impacts among categories or among the different signal models used in the analysis. In addition, the potential bias coming from non-resonant background modelling is shown relative to the background in the signal region $120<m_{\gamma \gamma}<130 \mathrm{GeV}$.

addition, the uncertainties in the scale and resolution of the $E_{\mathrm{T}}^{\text {miss }}$ soft term are estimated by using the method described in ref. [101]. The overall jet and $E_{\mathrm{T}}^{\text {miss }}$ uncertainties in the SM Higgs boson processes vary from $1.0 \%$ to $34 \%$ for each category and for different SM Higgs boson production processes. Overall, they amount to $0.4 \%-14 \%$ for the total SM Higgs boson background. For the signal processes, the overall jet and $E_{\mathrm{T}}^{\mathrm{miss}}$ uncertainties range from $0.2 \%$ to $3.3 \%$. An uncertainty in the pile-up modelling in MC simulation is accounted for. This results in an uncertainty of $0.3 \%-1.8 \%$ in the signal yield and $1.3 \%^{-}$ $1.5 \%$ in the SM Higgs boson yield. The uncertainties related to the $b$-tagging of jets are typically less than $1.5 \%$ in the SM Higgs boson yield used in the 'follow-up' analysis.

The predicted cross-sections of the SM Higgs boson and signal processes are affected by uncertainties due to missing higher-order terms in perturbative QCD. These uncertainties are estimated by varying the factorisation and renormalisation scales up and down from their nominal values by a factor of two, recalculating the cross-section in each case, and taking the largest deviation from the nominal cross-section as the uncertainty. The acceptance uncertainty related to the renormalisation and factorisation scales is less than 
$1 \%$ for the signal and 3.7\%-5.9\% for the SM Higgs boson processes [28]. The normalisation uncertainty of the SM Higgs boson processes is $1.7 \%$ to $2.8 \%$. For the signal processes, the effect of PDF and $\alpha_{\mathrm{S}}$ uncertainties in the acceptance times selection efficiency is below $6.6 \%$. It is estimated by using the recommendations of PDF4LHC [28]. Both the intra$\mathrm{PDF}$ and inter-PDF uncertainties are extracted. Intra-PDF uncertainties are obtained by varying the parameters of the NNPDF3.0LO PDF set, while inter-PDF uncertainties are estimated by using alternative PDF sets (CT14 [110] at LO and MMHT2014 [111] at LO). The final inter-PDF uncertainty is the maximum deviation among all the variations from the central value obtained using the NNPDF3.0LO PDF set. In the case of the SM Higgs boson processes, the acceptance effect of $\alpha_{\mathrm{S}}$ and the choice of PDFs ranges from $2.1 \%$ to $2.9 \%$, and its normalisation effect is $2.5 \%$ to $5.7 \%$. The uncertainty in the branching fraction of $h \rightarrow \gamma \gamma$ is $1.73 \%$ [28]. The uncertainty in the effect of multiple parton-parton interactions is estimated by switching them on and off in PутніA in the production of the ggF SM Higgs boson and signal samples. The resulting uncertainty in the number of events in this sample conservatively reaches $1 \%$ for all the categories.

\section{Results}

The results are derived from an unbinned likelihood fit to the $m_{\gamma \gamma}$ distributions in the range $105<m_{\gamma \gamma}<160 \mathrm{GeV}$ in each category simultaneously. The impact of the SM Higgs boson mass uncertainty is negligible. The signal strength and the background shape parameters are free parameters. The SM Higgs boson yields are taken from the SM predictions as discussed in section 3. The systematic uncertainty in each nuisance parameter is taken into account by multiplying the likelihood by a Gaussian penalty function centred on the nominal value of this parameter with a width set to its uncertainty. The nominal value of each SM Higgs boson background nuisance parameter (including its yield) is taken from the simulation normalised to the SM theoretical predictions.

Figures 4,5 and 6 show the $m_{\gamma \gamma}$ distribution as well as the analytical signal-plusbackground fits, for all 12 signal categories. The total background contains the nonresonant background and the predicted SM Higgs boson contribution. The fit results combining the $\tilde{\chi}_{1}^{ \pm} \tilde{\chi}_{2}^{0} \rightarrow W^{ \pm} \tilde{\chi}_{1}^{0} h \tilde{\chi}_{1}^{0}$ signal with $m\left(\tilde{\chi}_{1}^{ \pm} / \tilde{\chi}_{2}^{0}\right)=200 \mathrm{GeV}$ and $m\left(\tilde{\chi}_{1}^{0}\right)=0.5 \mathrm{GeV}$, SM Higgs boson and non-resonant background are shown as the solid curves. A small excess of around two standard deviations is seen in Category 4, however it is consistent with a statistical fluctuation of the SM prediction.

The event yields in the range $120<m_{\gamma \gamma}<130 \mathrm{GeV}$ for data, the signal models, the SM Higgs boson background and non-resonant background in the 12 categories are shown in table 4. The signal samples shown correspond to the $\tilde{\chi}_{1}^{ \pm} \tilde{\chi}_{2}^{0} \rightarrow W^{ \pm} \tilde{\chi}_{1}^{0} h \tilde{\chi}_{1}^{0}$ signal with $m\left(\tilde{\chi}_{1}^{ \pm} / \tilde{\chi}_{2}^{0}\right)=200 \mathrm{GeV}$ and $m\left(\tilde{\chi}_{1}^{0}\right)=0.5 \mathrm{GeV}$, and the $h \tilde{G} h \tilde{G}$ signal with $m\left(\tilde{\chi}_{1}^{0}\right)=150 \mathrm{GeV}$ and $m(\tilde{G})=1 \mathrm{MeV}$. The yields for the non-resonant background and the SM Higgs boson are obtained from a simultaneous background-only fit to the full $m_{\gamma \gamma}$ spectrum for the 12 categories. For the 'Leptonic' categories, the $W h$ process is the largest SM Higgs boson process and occupies $38 \%-55 \%$ of total events. The $t \bar{t} h$ events dominate in the 'Hadronic' categories, which account for $36 \%-41 \%$ of total SM Higgs boson process events. In the 


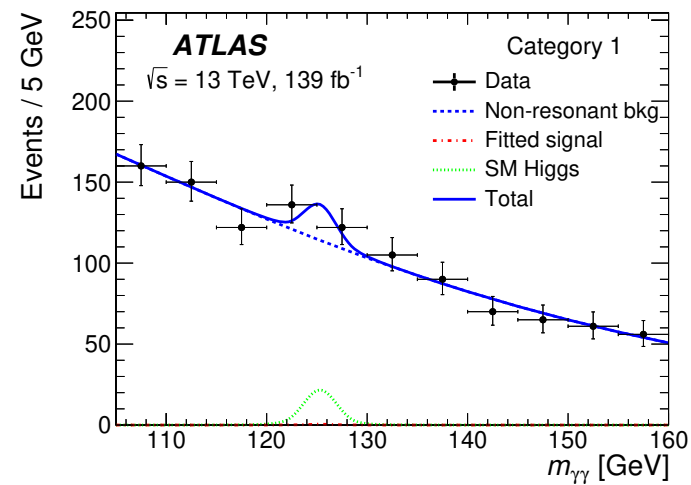

(a)

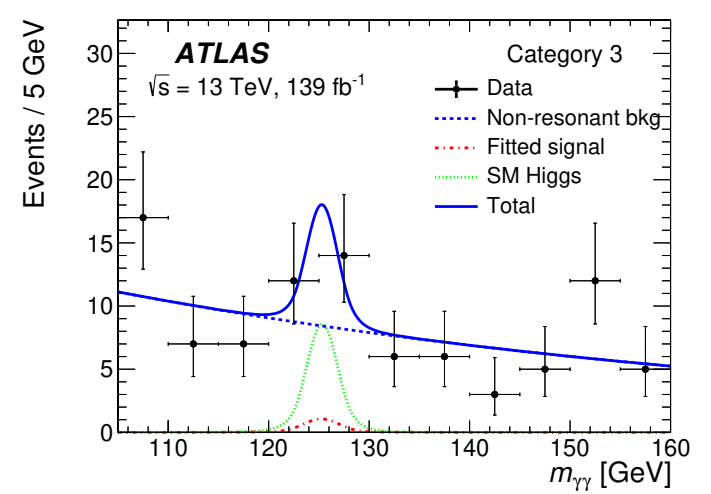

(c)

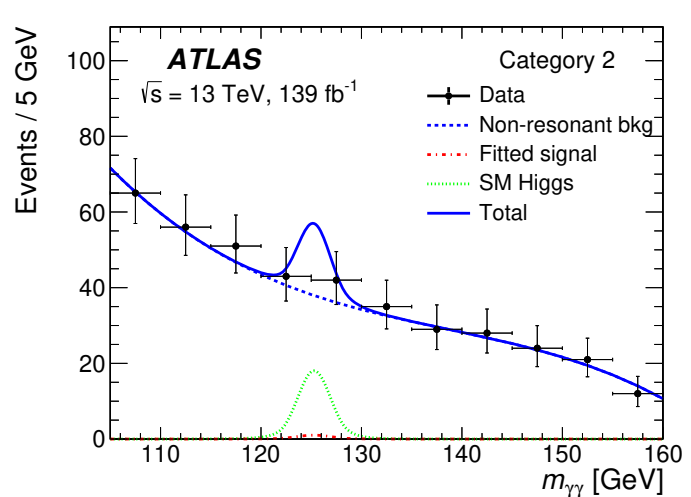

(b)

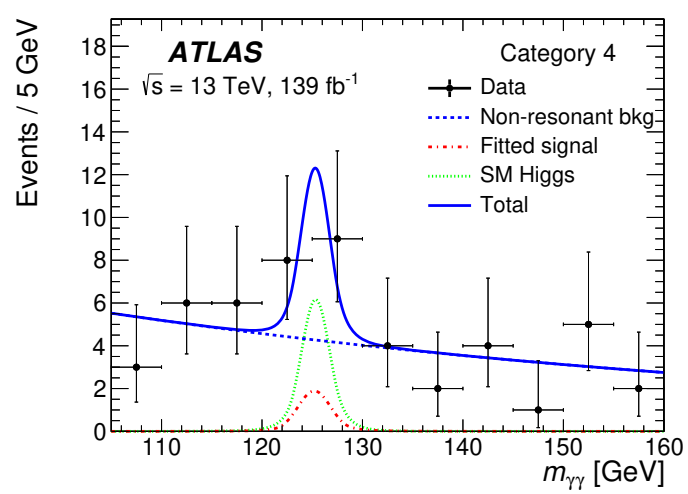

(d)

Figure 4. Diphoton invariant mass spectra and the corresponding fitted signal and background in the Leptonic categories (a) 1, (b) 2, (c) 3, and (d) 4. The signal samples shown correspond to the $\tilde{\chi}_{1}^{ \pm} \tilde{\chi}_{2}^{0} \rightarrow W^{ \pm} \tilde{\chi}_{1}^{0} h \tilde{\chi}_{1}^{0}$ signal with $m\left(\tilde{\chi}_{1}^{ \pm} / \tilde{\chi}_{2}^{0}\right)=200 \mathrm{GeV}$ and $m\left(\tilde{\chi}_{1}^{0}\right)=0.5 \mathrm{GeV}$. The non-resonant background (dashed curve), the SM Higgs boson (dotted curve), and the signal (dash-dotted curve) are obtained from a simultaneous signal-plus-background fit to the full $m_{\gamma \gamma}$ spectrum for the 12 categories. The total of these contributions is shown by the solid curves.

'Rest' categories, events from the $Z h$ process dominates and holds $37 \%-58 \%$ of total SM Higgs boson contribution. The yields for the signals are estimated from the simulation and normalized to the NLO+NLL predicted cross-sections. The uncertainties correspond to the statistical and systematic uncertainties summed in quadrature. For all the categories, data and background predictions agree within the statistical and systematic uncertainties.

The independently fitted $m_{\gamma \gamma}$ distributions for the 'follow-up' signal regions are shown in figure 7. No significant excess of events is seen in either of the two regions. In 'SR1L $\gamma \gamma$ a', two events are observed with $3.1 \pm 0.8$ non-resonant background events and $0.5_{-0.4}^{+0.2} \mathrm{SM}$ Higgs boson events expected in the range $120<m_{\gamma \gamma}<130 \mathrm{GeV}$. In the case of 'SR1L $\gamma \gamma$ b', 31 events are observed, whereas $16.6 \pm 1.9$ events from non-resonant background and $8.6_{-2.1}^{+1.3}$ events from the SM Higgs boson are expected in the range $120<m_{\gamma \gamma}<130 \mathrm{GeV}$.

\subsection{Limits on the visible cross-section}

The observed yields agree with the background predictions, as shown in table 4, and no significant excess of events is observed. Upper limits are set on the visible cross-section 


\begin{tabular}{|r|rccccc|}
\hline Category & Data & Total bkg. & Non-resonant bkg. & SM Higgs boson & $W^{ \pm} \tilde{\chi}_{1}^{0} h \tilde{\chi}_{1}^{0}$ & $h \tilde{G} h \tilde{G}$ \\
\hline 1 & 258 & $246 \pm 7$ & $230 \pm 7$ & $16.3 \pm 1.4$ & $2.8 \pm 0.6$ & $13 \pm 6$ \\
2 & 85 & $93 \pm 4$ & $77 \pm 4$ & $15.6 \pm 1.3$ & $6.6 \pm 1.5$ & $16 \pm 7$ \\
3 & 26 & $24.1 \pm 2.0$ & $17.1 \pm 1.9$ & $7.0 \pm 0.6$ & $6.9 \pm 1.5$ & $6.5 \pm 2.7$ \\
4 & 17 & $12.8 \pm 1.4$ & $8.4 \pm 1.3$ & $4.4 \pm 0.4$ & $10.7 \pm 2.4$ & $3.8 \pm 1.6$ \\
5 & 54 & $60 \pm 4$ & $57.9 \pm 3.5$ & $1.9 \pm 0.6$ & $7.2 \pm 1.6$ & $3.3 \pm 1.4$ \\
6 & 11 & $16.1 \pm 1.8$ & $15.4 \pm 1.8$ & $0.74 \pm 0.26$ & $6.0 \pm 1.3$ & $1.6 \pm 0.7$ \\
7 & 8 & $6.3 \pm 1.1$ & $5.9 \pm 1.1$ & $0.42 \pm 0.10$ & $4.3 \pm 1.0$ & $0.71 \pm 0.34$ \\
8 & 4 & $5.2 \pm 1.0$ & $4.4 \pm 1.0$ & $0.80 \pm 0.11$ & $5.3 \pm 1.2$ & $0.76 \pm 0.33$ \\
9 & 71 & $69 \pm 4$ & $65 \pm 4$ & $3.9 \pm 0.8$ & $9.1 \pm 2.0$ & $3.1 \pm 1.3$ \\
10 & 29 & $26.3 \pm 2.2$ & $24.2 \pm 2.2$ & $2.1 \pm 0.4$ & $6.9 \pm 1.5$ & $1.8 \pm 0.8$ \\
11 & 6 & $8.6 \pm 1.2$ & $7.2 \pm 1.2$ & $1.40 \pm 0.22$ & $4.6 \pm 1.0$ & $1.1 \pm 0.5$ \\
12 & 22 & $16.6 \pm 1.7$ & $13.4 \pm 1.7$ & $3.15 \pm 0.33$ & $7.9 \pm 1.8$ & $1.7 \pm 0.7$ \\
\hline
\end{tabular}

Table 4. Event yields in the range $120<m_{\gamma \gamma}<130 \mathrm{GeV}$ for data, the signal models, the SM Higgs boson background and non-resonant background in each analysis category, for an integrated luminosity of $139 \mathrm{fb}^{-1}$. The signal samples shown correspond to the $\tilde{\chi}_{1}^{ \pm} \tilde{\chi}_{2}^{0} \rightarrow W^{ \pm} \tilde{\chi}_{1}^{0} h \tilde{\chi}_{1}^{0}$ signal with $m\left(\tilde{\chi}_{1}^{ \pm} / \tilde{\chi}_{2}^{0}\right)=200 \mathrm{GeV}$ and $m\left(\tilde{\chi}_{1}^{0}\right)=0.5 \mathrm{GeV}$, and the $h \tilde{G} h \tilde{G}$ signals with $m\left(\tilde{\chi}_{1}^{0}\right)=150 \mathrm{GeV}$ and $m(\tilde{G})=1 \mathrm{MeV}$. The yields for the non-resonant background and SM Higgs boson are obtained from a simultaneous background-only fit to the full $m_{\gamma \gamma}$ spectrum for the 12 categories. The yields for the signals are estimated from the simulation. The uncertainties correspond to the statistical and systematic uncertainties summed in quadrature.

$\sigma_{\text {vis }}^{\text {BSM }} \equiv(\mathcal{A} \times \epsilon \times \sigma)^{\text {BSM }}$ for BSM physics processes producing $E_{\mathrm{T}}^{\text {miss }}$ and an SM Higgs boson decaying into two photons, where $\mathcal{A}$ and $\epsilon$ are the acceptance and the efficiency for the signal, respectively. The limits are extracted by performing a fit to the non-resonant background and SM Higgs boson background, individually for each category, each time injecting a signal with the same mass distribution as the SM Higgs boson but with a free normalisation. Figure 8 shows the observed and expected 95\% confidence level (CL) upper limits on $\sigma_{\text {vis }}^{\text {BSM }}$ for each of the 12 different categories, which are calculated using a one-sided profile-likelihood ratio and the $\mathrm{CL}_{\mathrm{s}}$ formalism [112] with the asymptotic approximation described in ref. [113]. The statistical uncertainty is dominant for all categories.

\subsection{Interpretation of the wino-like $\tilde{\chi}_{1}^{ \pm} \tilde{\chi}_{2}^{0} \rightarrow W^{ \pm} \tilde{\chi}_{1}^{0} h \tilde{\chi}_{1}^{0}$ model}

Since no significant excess is observed, fit results are interpreted in terms of $95 \%$ CL exclusion limits on the production cross-section of the wino-like $\tilde{\chi}_{1}^{ \pm} \tilde{\chi}_{2}^{0} \rightarrow W^{ \pm} \tilde{\chi}_{1}^{0} h \tilde{\chi}_{1}^{0}$ model $[26,27]$. Upper limits on the contribution of events from the considered processes are computed by using the modified frequentist $\mathrm{CL}_{\mathrm{s}}$ approach based on asymptotic formulae $[112,113]$. Figure 9 shows $95 \%$ CL exclusion limits on the production cross-section of $\tilde{\chi}_{1}^{ \pm} \tilde{\chi}_{2}^{0} \rightarrow W^{ \pm} \tilde{\chi}_{1}^{0} h \tilde{\chi}_{1}^{0}$ as a function of $m\left(\tilde{\chi}_{1}^{ \pm} / \tilde{\chi}_{2}^{0}\right)$. The observed $95 \%$ CL upper limits on the production cross-section vary from $1.92 \mathrm{pb}$ to $0.16 \mathrm{pb}$ for $m\left(\tilde{\chi}_{1}^{ \pm} / \tilde{\chi}_{2}^{0}\right)$ from $150 \mathrm{GeV}$ to $600 \mathrm{GeV}$. The expected $95 \% \mathrm{CL}$ upper limits range from $1.43 \mathrm{pb}$ to $0.11 \mathrm{pb}$ for the same range. A $95 \% \mathrm{CL}$ lower limit of $310 \mathrm{GeV}$ in $m\left(\tilde{\chi}_{1}^{ \pm} / \tilde{\chi}_{2}^{0}\right)$, where $m\left(\tilde{\chi}_{1}^{0}\right)=0.5 \mathrm{GeV}$, is set. 


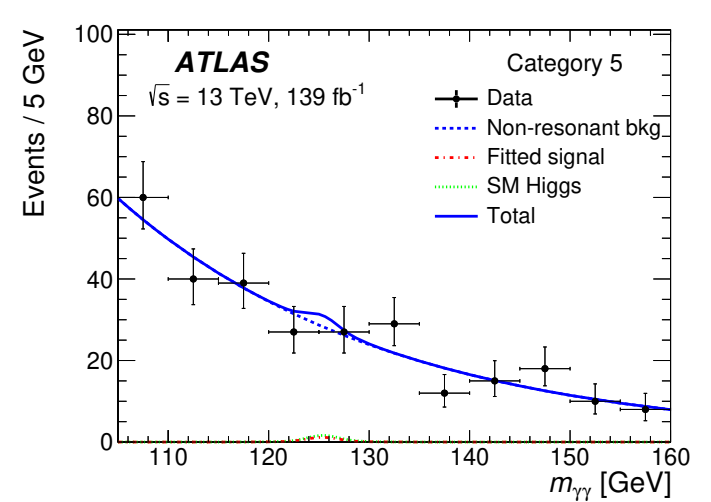

(a)

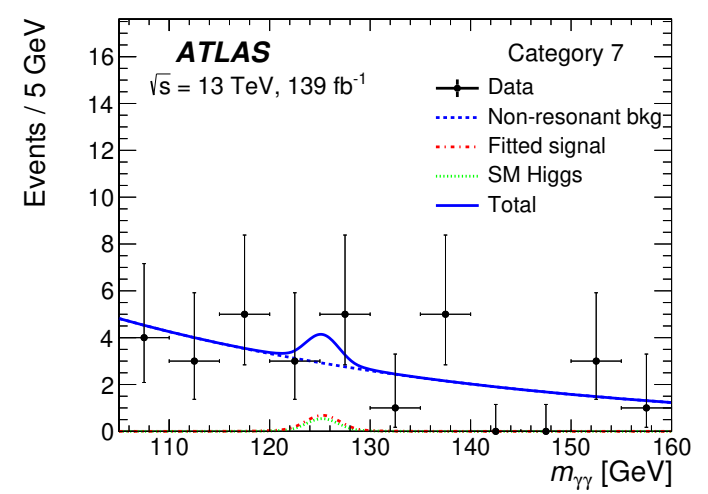

(c)

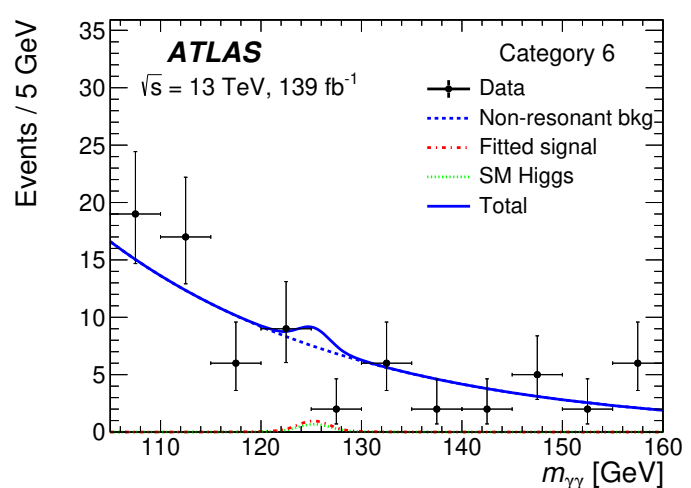

(b)

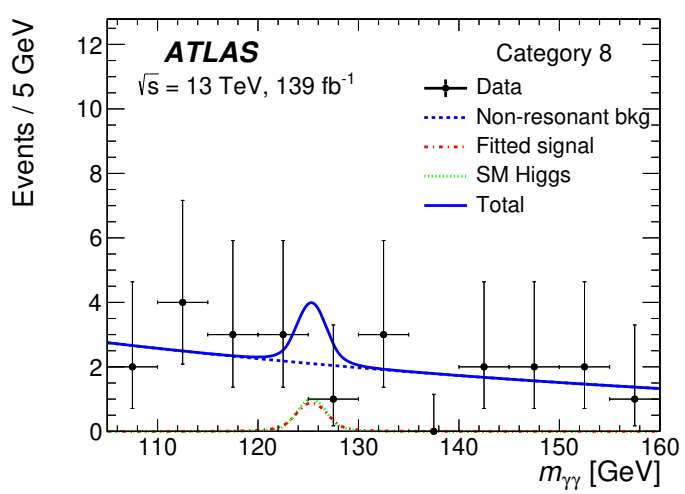

(d)

Figure 5. Diphoton invariant mass spectra and the corresponding fitted signal and background in the Hadronic categories (a) 5, (b) 6, (c) 7, and (d) 8. The signal samples shown correspond to the $\tilde{\chi}_{1}^{ \pm} \tilde{\chi}_{2}^{0} \rightarrow W^{ \pm} \tilde{\chi}_{1}^{0} h \tilde{\chi}_{1}^{0}$ signal with $m\left(\tilde{\chi}_{1}^{ \pm} / \tilde{\chi}_{2}^{0}\right)=200 \mathrm{GeV}$ and $m\left(\tilde{\chi}_{1}^{0}\right)=0.5 \mathrm{GeV}$. The non-resonant background (dashed curve), the SM Higgs boson (dotted curve), and the signal (dash-dotted curve) are obtained from a simultaneous signal-plus-background fit to the full $m_{\gamma \gamma}$ spectrum for the 12 categories. The total of these contributions is shown by the solid curves.

The observed and expected exclusion contours at 95\% CL for the $\tilde{\chi}_{1}^{ \pm} \tilde{\chi}_{2}^{0}$ production in the $m\left(\tilde{\chi}_{1}^{ \pm} / \tilde{\chi}_{2}^{0}\right)-m\left(\tilde{\chi}_{1}^{0}\right)$ plane are shown in figure 10 .

\subsection{Interpretation of the higgsino-like $h \tilde{G} h \tilde{G}$ model}

As a second SUSY scenario, a GMSB model where the two lightest neutralinos and the lightest chargino are higgsinos is considered [36-38]. The $\tilde{\chi}_{1}^{ \pm}, \tilde{\chi}_{1}^{0}$ and $\tilde{\chi}_{2}^{0}$ are almost mass degenerate in this model, with $\tilde{\chi}_{1}^{0}$ being the lightest of the three states. The LSP is a gravitino. In figure 11, the observed and expected 95\% CL upper limits, with uncertainties, on the higgsino production cross-section in the $h \tilde{G} h \tilde{G}$ models for different $m\left(\tilde{\chi}_{1}^{0}\right)$ masses are presented. The levelling off of expected limits at low $m\left(\tilde{\chi}_{1}^{0}\right)$ masses is due to the acceptance times efficiency in this region. The theoretical prediction includes the $\tilde{\chi}_{1}^{0} \tilde{\chi}_{2}^{0}, \tilde{\chi}_{1}^{0} \tilde{\chi}_{1}^{ \pm}, \tilde{\chi}_{2}^{0} \tilde{\chi}_{1}^{ \pm}$, and $\tilde{\chi}_{1}^{ \pm} \tilde{\chi}_{1}^{\mp}$ production modes, where $\tilde{\chi}_{1}^{ \pm}$and $\tilde{\chi}_{2}^{0}$ promptly decay into the $\tilde{\chi}_{1}^{0}$ and particles that have too low momentum to be detected. In the $h \tilde{G} h \tilde{G}$ model, higgsino masses below $380 \mathrm{GeV}$ are excluded at $95 \% \mathrm{CL}$. 


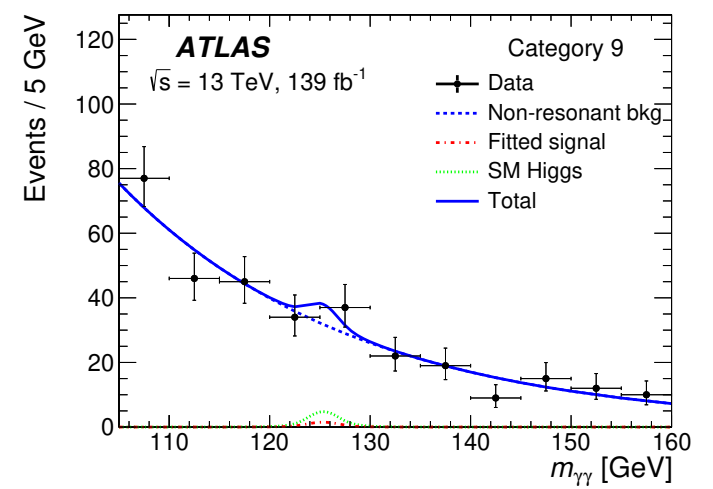

(a)

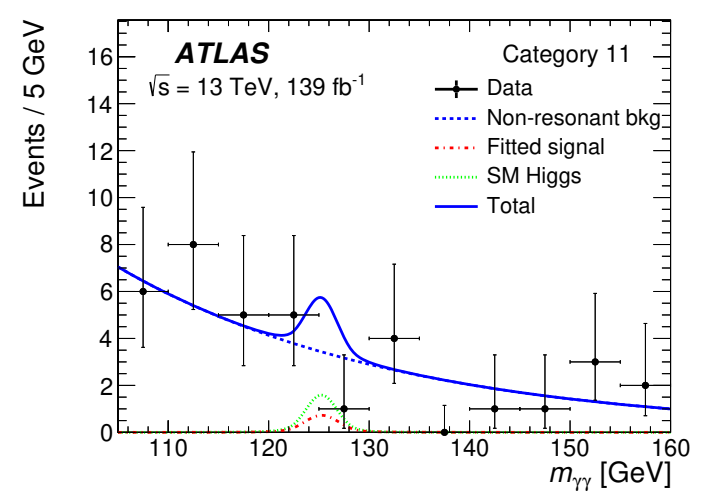

(c)

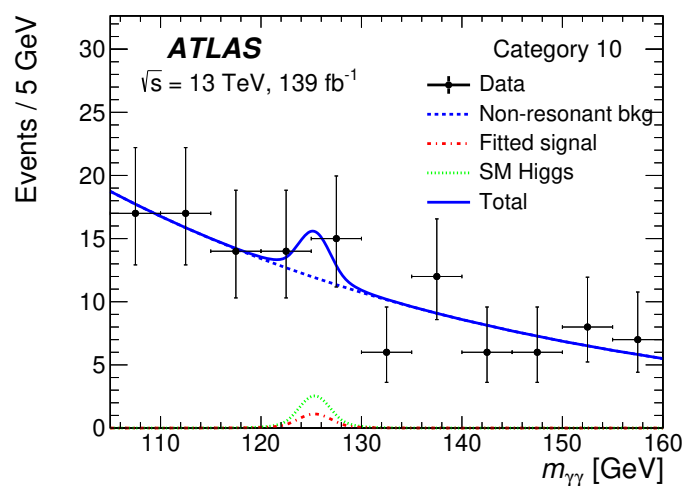

(b)

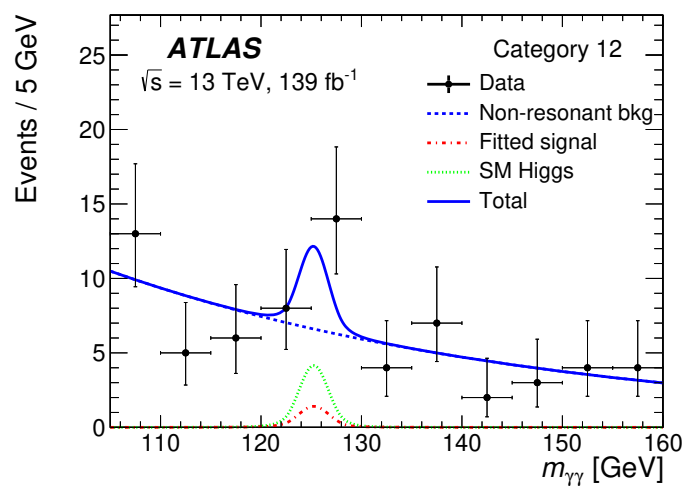

(d)

Figure 6. Diphoton invariant mass spectra and the corresponding fitted signal and background in the Rest categories (a) 9, (b) 10, (c) 11, and (d) 12. The signal samples shown correspond to the $\tilde{\chi}_{1}^{ \pm} \tilde{\chi}_{2}^{0} \rightarrow W^{ \pm} \tilde{\chi}_{1}^{0} h \tilde{\chi}_{1}^{0}$ signal with $m\left(\tilde{\chi}_{1}^{ \pm} / \tilde{\chi}_{2}^{0}\right)=200 \mathrm{GeV}$ and $m\left(\tilde{\chi}_{1}^{0}\right)=0.5 \mathrm{GeV}$. The non-resonant background (dashed curve), the SM Higgs boson (dotted curve), and the signal (dash-dotted curve) are obtained from a simultaneous signal-plus-background fit to the full $m_{\gamma \gamma}$ spectrum for the 12 categories. The total of these contributions is shown by the solid curves.

\section{Conclusion}

A search for a chargino and a neutralino decaying via the $125 \mathrm{GeV}$ Higgs boson into photons is presented. This study is based on the full data collected between 2015 and 2018 with the ATLAS detector at the LHC, corresponding to an integrated luminosity of $139 \mathrm{fb}^{-1}$ of $p p$ collisions at a centre-of-mass energy of $13 \mathrm{TeV}$. No significant excess over the expected background is observed. Upper limits at $95 \%$ confidence level are set on the $\tilde{\chi}_{1}^{ \pm} \tilde{\chi}_{2}^{0}$ and higgsino production cross-section, and the visible cross-section for beyond the Standard Model physics processes. For the $\tilde{\chi}_{1}^{ \pm} \tilde{\chi}_{2}^{0} \rightarrow W^{ \pm} \tilde{\chi}_{1}^{0} h \tilde{\chi}_{1}^{0}$ model, the observed $95 \%$ confidencelevel upper limits on the production cross-section vary from $1.92 \mathrm{pb}$ to $0.16 \mathrm{pb}$ for $m\left(\tilde{\chi}_{1}^{ \pm} / \tilde{\chi}_{2}^{0}\right)$ from $150 \mathrm{GeV}$ to $600 \mathrm{GeV}$, where $m\left(\tilde{\chi}_{1}^{0}\right)$ is set to $0.5 \mathrm{GeV}$. The expected $95 \%$ confidence-level upper limits range from $1.43 \mathrm{pb}$ to $0.11 \mathrm{pb}$ for the same mass interval. A $95 \%$ confidencelevel lower limit of $310 \mathrm{GeV}$ in $m\left(\tilde{\chi}_{1}^{ \pm} / \tilde{\chi}_{2}^{0}\right)$, where $m\left(\tilde{\chi}_{1}^{0}\right)=0.5 \mathrm{GeV}$, is set. Upper limits at the $95 \%$ confidence-level are set on the higgsino production cross-section. Higgsino masses 


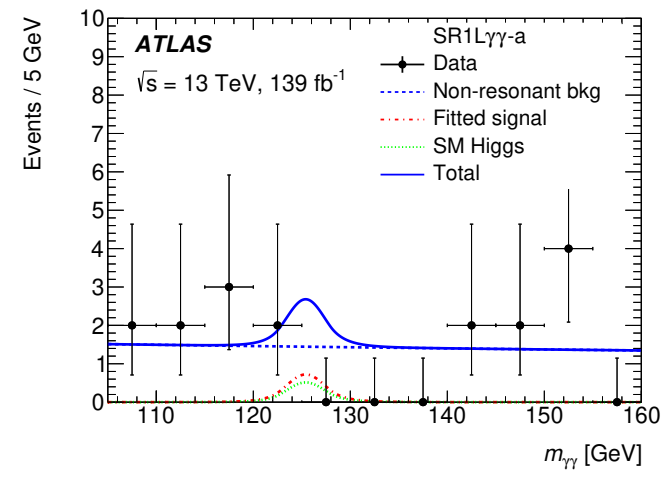

(a)

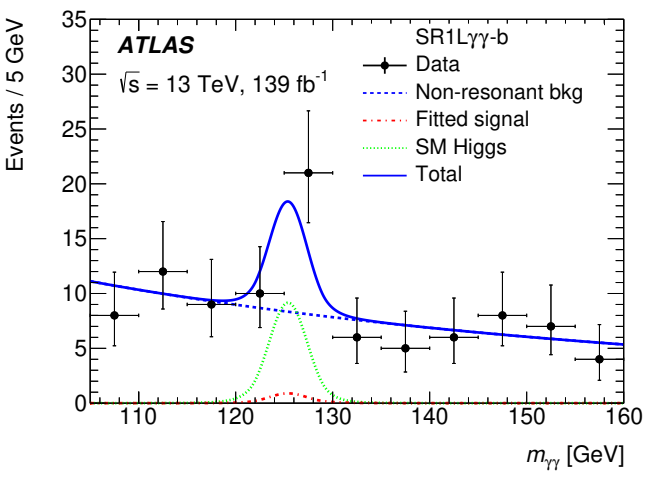

(b)

Figure 7. Diphoton invariant mass spectra and the corresponding fitted signal and background in the signal regions (a) 'SR1L $\gamma \gamma-\mathrm{a}$ ' and (b) 'SR1L $\gamma \gamma$-b'. The signal samples shown correspond to the $\tilde{\chi}_{1}^{ \pm} \tilde{\chi}_{2}^{0} \rightarrow W^{ \pm} \tilde{\chi}_{1}^{0} h \tilde{\chi}_{1}^{0}$ signal with $m\left(\tilde{\chi}_{1}^{ \pm} / \tilde{\chi}_{2}^{0}\right)=200 \mathrm{GeV}$ and $m\left(\tilde{\chi}_{1}^{0}\right)=0.5 \mathrm{GeV}$. The non-resonant background (dashed curve), the SM Higgs boson (dotted curve), and the signal (dash-dotted curve) are obtained from a signal-plus-background fit to the full $m_{\gamma \gamma}$ spectrum in 'SR1L $\gamma \gamma$-a' (a) and 'SR1L $\gamma \gamma$-b' (b) separately. The total of these contributions is shown by the solid curves.

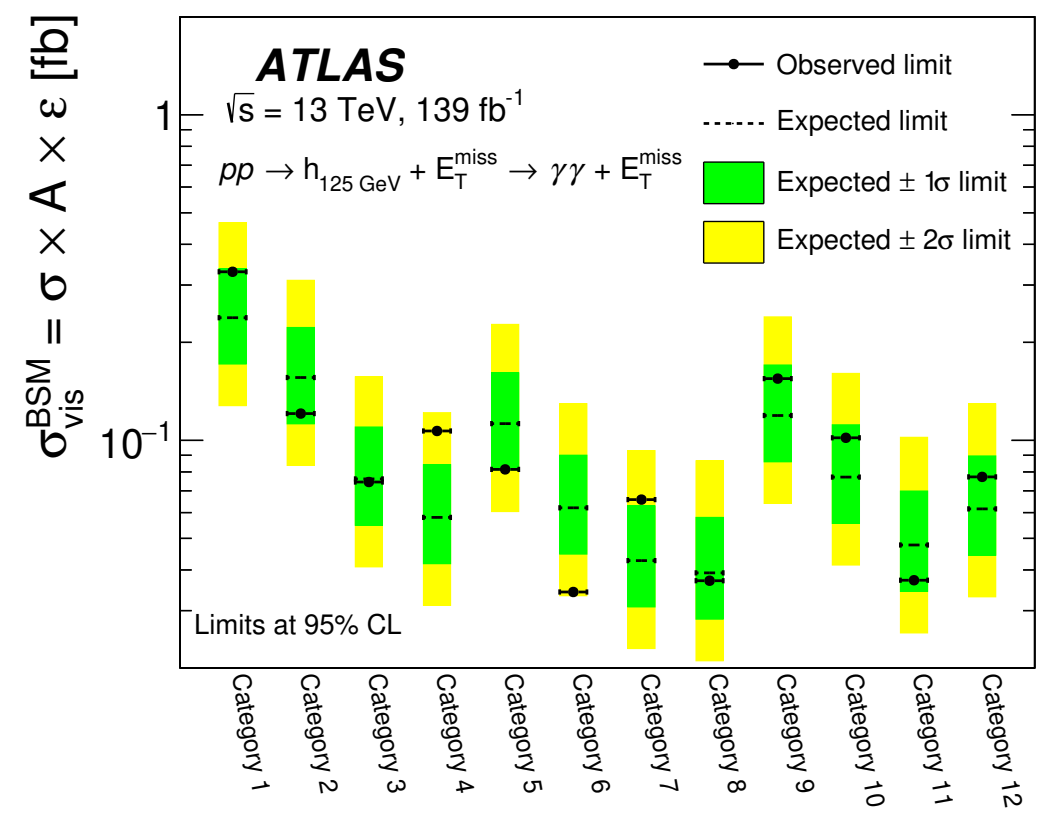

Figure 8. The 95\% CL model-independent upper limits computed from individual fits in each of 12 categories on the visible cross-section $\sigma_{\mathrm{vis}}^{\mathrm{BSM}}=\sigma \times A \times \epsilon$ for any $p p \rightarrow h+E_{\mathrm{T}}^{\text {miss }} \rightarrow \gamma \gamma+E_{\mathrm{T}}^{\text {miss }}$ BSM processes. 


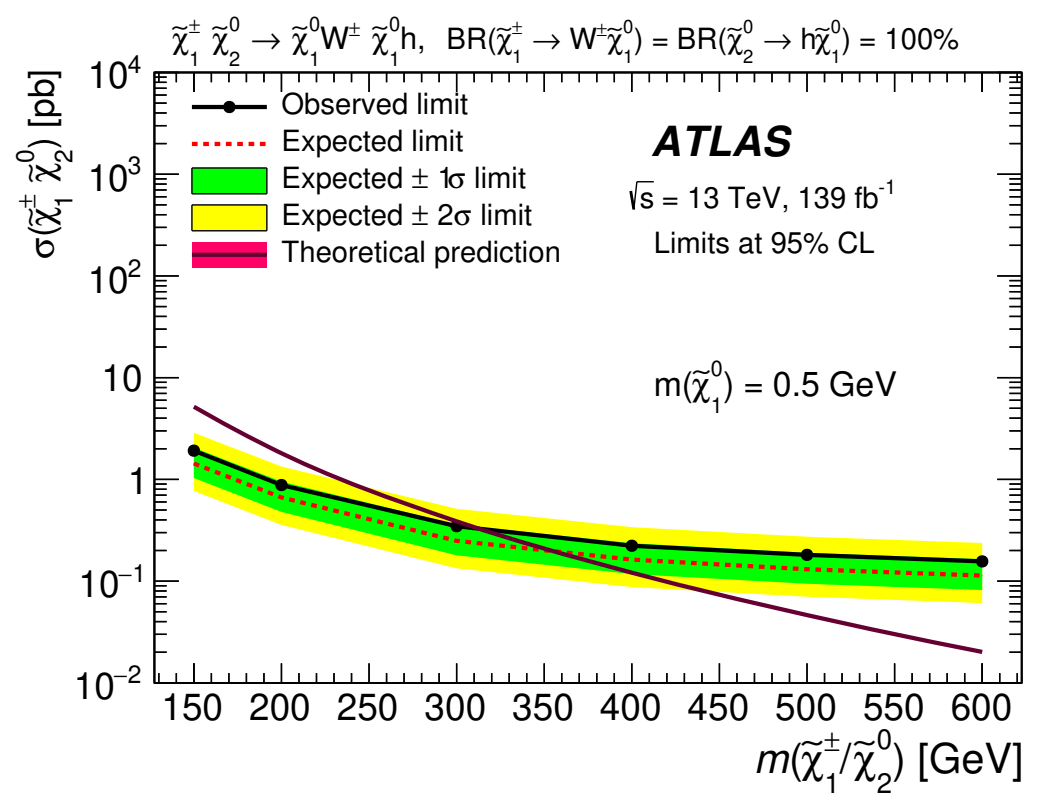

Figure 9. Expected and observed 95\% CL exclusion upper limits on the production cross-section of $\tilde{\chi}_{1}^{ \pm} \tilde{\chi}_{2}^{0} \rightarrow W^{ \pm} \tilde{\chi}_{1}^{0} h \tilde{\chi}_{1}^{0}$ as a function of $m\left(\tilde{\chi}_{1}^{ \pm} / \tilde{\chi}_{2}^{0}\right)$.

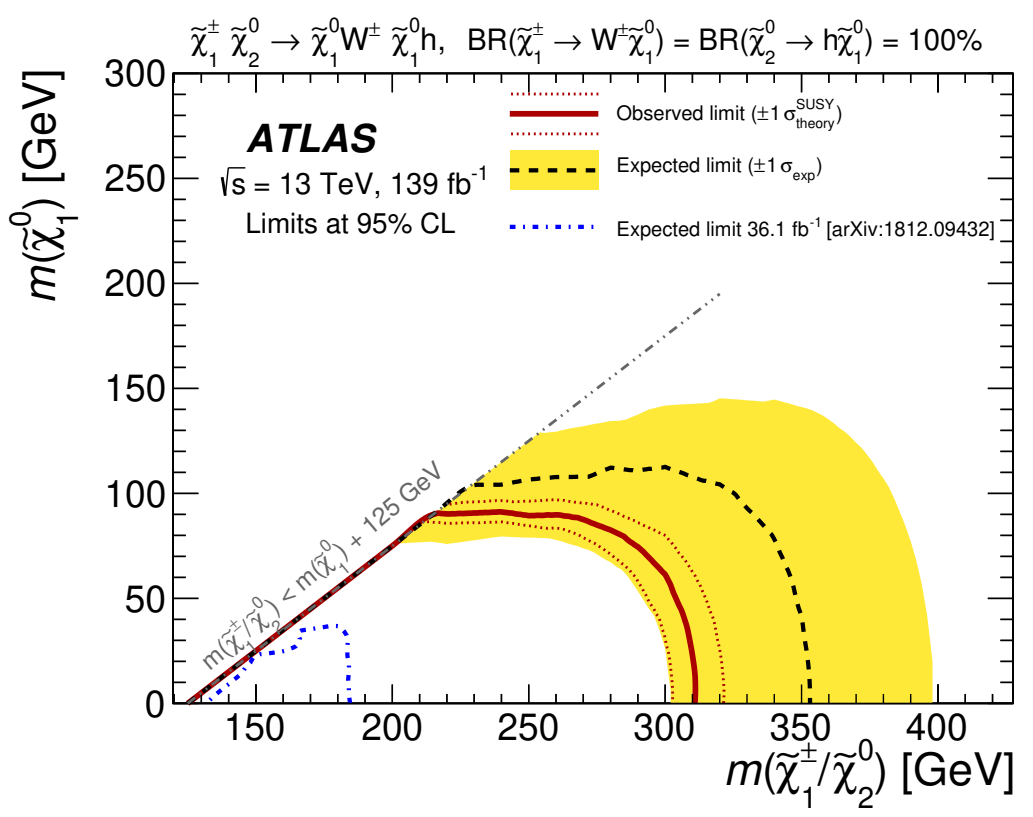

Figure 10. The observed (solid line) and expected (dashed lines) exclusion limit contours at $95 \%$ CL for the $\tilde{\chi}_{1}^{ \pm} \tilde{\chi}_{2}^{0}$ production in the $m\left(\tilde{\chi}_{1}^{ \pm} / \tilde{\chi}_{2}^{0}\right)-m\left(\tilde{\chi}_{1}^{0}\right)$ plane. The dotted lines represent the $\pm 1 \sigma$ theoretical uncertainty for the observed limit. The $\pm 1 \sigma$ expected exclusion limit contour is shown as the shaded band. The expected limit for the $36.1 \mathrm{fb}^{-1}$ analysis [32] is also shown for comparison in the dash-dotted line. 


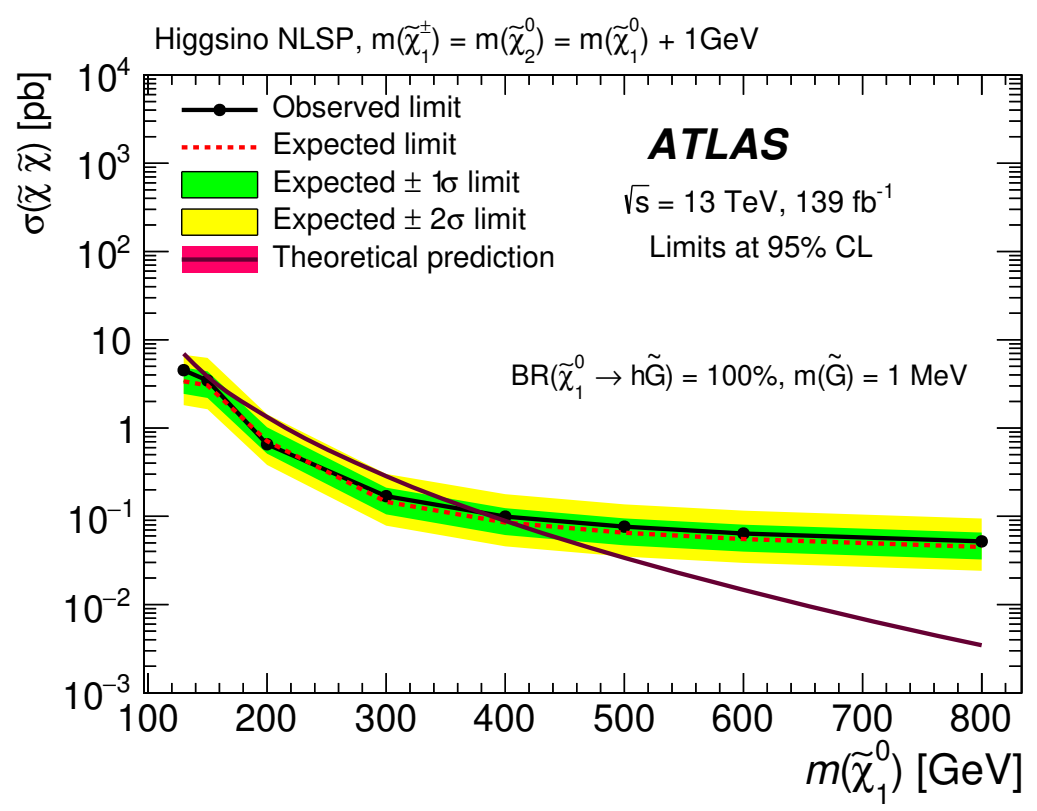

Figure 11. Expected and observed 95\% CL exclusion upper limits on the higgsino production $\left(\tilde{\chi} \tilde{\chi} \equiv \tilde{\chi}_{1}^{0} \tilde{\chi}_{2}^{0}, \tilde{\chi}_{1}^{0} \tilde{\chi}_{1}^{ \pm}, \tilde{\chi}_{2}^{0} \tilde{\chi}_{1}^{ \pm}, \tilde{\chi}_{1}^{ \pm} \tilde{\chi}_{1}^{\mp}\right)$ cross-section for the $h \tilde{G} h \tilde{G}$ signal model. As a function of the higgsino mass. The theoretical prediction includes the $\tilde{\chi}_{1}^{0} \tilde{\chi}_{2}^{0}, \tilde{\chi}_{1}^{0} \tilde{\chi}_{1}^{ \pm}, \tilde{\chi}_{2}^{0} \tilde{\chi}_{1}^{ \pm}$, and $\tilde{\chi}_{1}^{ \pm} \tilde{\chi}_{1}^{\mp}$ production modes, where $\tilde{\chi}_{1}^{ \pm}$and $\tilde{\chi}_{2}^{0}$ promptly decay into the $\tilde{\chi}_{1}^{0}$ and particles that have too low momentum to be detected.

below $380 \mathrm{GeV}$ are excluded for the case of the higgsino fully decaying into a Higgs boson and a gravitino.

\section{Acknowledgments}

We thank CERN for the very successful operation of the LHC, as well as the support staff from our institutions without whom ATLAS could not be operated efficiently.

We acknowledge the support of ANPCyT, Argentina; YerPhI, Armenia; ARC, Australia; BMWFW and FWF, Austria; ANAS, Azerbaijan; SSTC, Belarus; CNPq and FAPESP, Brazil; NSERC, NRC and CFI, Canada; CERN; CONICYT, Chile; CAS, MOST and NSFC, China; COLCIENCIAS, Colombia; MSMT CR, MPO CR and VSC CR, Czech Republic; DNRF and DNSRC, Denmark; IN2P3-CNRS and CEA-DRF/IRFU, France; SRNSFG, Georgia; BMBF, HGF and MPG, Germany; GSRT, Greece; RGC and Hong Kong SAR, China; ISF and Benoziyo Center, Israel; INFN, Italy; MEXT and JSPS, Japan; CNRST, Morocco; NWO, Netherlands; RCN, Norway; MNiSW and NCN, Poland; FCT, Portugal; MNE/IFA, Romania; MES of Russia and NRC KI, Russia Federation; JINR; MESTD, Serbia; MSSR, Slovakia; ARRS and MIZŠ, Slovenia; DST/NRF, South Africa; MINECO, Spain; SRC and Wallenberg Foundation, Sweden; SERI, SNSF and Cantons of Bern and Geneva, Switzerland; MOST, Taiwan; TAEK, Turkey; STFC, United Kingdom; DOE and NSF, United States of America. In addition, individual groups and members have received support from BCKDF, CANARIE, Compute Canada and CRC, Canada; ERC, 
ERDF, Horizon 2020, Marie Skłodowska-Curie Actions and COST, European Union; Investissements d'Avenir Labex, Investissements d'Avenir Idex and ANR, France; DFG and AvH Foundation, Germany; Herakleitos, Thales and Aristeia programmes co-financed by EU-ESF and the Greek NSRF, Greece; BSF-NSF and GIF, Israel; CERCA Programme Generalitat de Catalunya and PROMETEO Programme Generalitat Valenciana, Spain; Göran Gustafssons Stiftelse, Sweden; The Royal Society and Leverhulme Trust, United Kingdom.

The crucial computing support from all WLCG partners is acknowledged gratefully, in particular from CERN, the ATLAS Tier-1 facilities at TRIUMF (Canada), NDGF (Denmark, Norway, Sweden), CC-IN2P3 (France), KIT/GridKA (Germany), INFN-CNAF (Italy), NL-T1 (Netherlands), PIC (Spain), ASGC (Taiwan), RAL (U.K.) and BNL (U.S.A.), the Tier-2 facilities worldwide and large non-WLCG resource providers. Major contributors of computing resources are listed in ref. [114].

Open Access. This article is distributed under the terms of the Creative Commons Attribution License (CC-BY 4.0), which permits any use, distribution and reproduction in any medium, provided the original author(s) and source are credited.

\section{References}

[1] ATLAS collaboration, Observation of a new particle in the search for the Standard Model Higgs boson with the ATLAS detector at the LHC, Phys. Lett. B 716 (2012) 1 [arXiv: 1207.7214] [INSPIRE].

[2] CMS collaboration, Observation of a New Boson at a Mass of $125 \mathrm{GeV}$ with the CMS Experiment at the LHC, Phys. Lett. B 716 (2012) 30 [arXiv:1207.7235] [INSPIRE].

[3] ATLAS and CMS collaborations, Combined Measurement of the Higgs Boson Mass in pp Collisions at $\sqrt{s}=7$ and 8 TeV with the ATLAS and CMS Experiments, Phys. Rev. Lett. 114 (2015) 191803 [arXiv: 1503.07589] [INSPIRE].

[4] ATLAS and CMS collaborations, Measurements of the Higgs boson production and decay rates and constraints on its couplings from a combined ATLAS and CMS analysis of the LHC pp collision data at $\sqrt{s}=7$ and 8 TeV, JHEP 08 (2016) 045 [arXiv:1606.02266] [INSPIRE].

[5] N. Sakai, Naturalness in Supersymmetric Guts, Z. Phys. C 11 (1981) 153 [InSPIRE].

[6] S. Dimopoulos, S. Raby and F. Wilczek, Supersymmetry and the Scale of Unification, Phys. Rev. D 24 (1981) 1681 [INSPIRE].

[7] L.E. Ibáñez and G.G. Ross, Low-Energy Predictions in Supersymmetric Grand Unified Theories, Phys. Lett. B 105 (1981) 439 [INSPIRE].

[8] S. Dimopoulos and H. Georgi, Softly broken supersymmetry and SU(5), Nucl. Phys. B 193 (1981) 150 [INSPIRE].

[9] Y. Golfand and E.P. Likhtman, Extension of the Algebra of Poincaré Group Generators and Violation of p Invariance, JETP Lett. 13 (1971) 323 [INSPIRE].

[10] D.V. Volkov and V.P. Akulov, Is the Neutrino a Goldstone Particle?, Phys. Lett. B 46 (1973) 109 [INSPIRE]. 
[11] J. Wess and B. Zumino, Supergauge Transformations in Four-Dimensions, Nucl. Phys. B 70 (1974) 39 [INSPIRE].

[12] J. Wess and B. Zumino, Supergauge Invariant Extension of Quantum Electrodynamics, Nucl. Phys. B 78 (1974) 1 [inSPIRE].

[13] S. Ferrara and B. Zumino, Supergauge invariant Yang-Mills theories, Nucl. Phys. B 79 (1974) 413 [INSPIRE].

[14] A. Salam and J.A. Strathdee, Supersymmetry and Nonabelian Gauges, Phys. Lett. B 51 (1974) 353 [INSPIRE].

[15] H. Goldberg, Constraint on the Photino Mass from Cosmology, Phys. Rev. Lett. 50 (1983) 1419 [Erratum ibid. 103 (2009) 099905] [INSPIRE].

[16] J.R. Ellis, J.S. Hagelin, D.V. Nanopoulos, K.A. Olive and M. Srednicki, Supersymmetric Relics from the Big Bang, Nucl. Phys. B 238 (1984) 453 [inSPIRE].

[17] G.R. Farrar and P. Fayet, Phenomenology of the Production, Decay, and Detection of New Hadronic States Associated with Supersymmetry, Phys. Lett. B 76 (1978) 575 [INSPIRE].

[18] R. Barbieri and G.F. Giudice, Upper Bounds on Supersymmetric Particle Masses, Nucl. Phys. B 306 (1988) 63 [INSPIRE].

[19] B. de Carlos and J.A. Casas, One loop analysis of the electroweak breaking in supersymmetric models and the fine tuning problem, Phys. Lett. B 309 (1993) 320 [hep-ph/9303291] [INSPIRE].

[20] P. Fayet, Supersymmetry and Weak, Electromagnetic and Strong Interactions, Phys. Lett. B 64 (1976) 159 [INSPIRE].

[21] P. Fayet, Spontaneously Broken Supersymmetric Theories of Weak, Electromagnetic and Strong Interactions, Phys. Lett. B 69 (1977) 489 [InSPIRE].

[22] S.P. Das, M. Guchait and D.P. Roy, Testing SUSY models for the muon $g-2$ anomaly via chargino-neutralino pair production at the LHC, Phys. Rev. D 90 (2014) 055011 [arXiv: 1406.6925] [INSPIRE].

[23] M.A. Ajaib, B. Dutta, T. Ghosh, I. Gogoladze and Q. Shafi, Neutralinos and sleptons at the LHC in light of muon $(g-2)_{\mu}$, Phys. Rev. D 92 (2015) 075033 [arXiv:1505.05896] [INSPIRE].

[24] M. Chakraborti, U. Chattopadhyay, A. Choudhury, A. Datta and S. Poddar, Reduced LHC constraints for higgsino-like heavier electroweakinos, JHEP 11 (2015) 050 [arXiv: 1507.01395] [INSPIRE].

[25] M. Endo, K. Hamaguchi, S. Iwamoto and K. Yanagi, Probing minimal SUSY scenarios in the light of muon $g-2$ and dark matter, JHEP 06 (2017) 031 [arXiv:1704.05287] [INSPIRE].

[26] J. Alwall, P. Schuster and N. Toro, Simplified Models for a First Characterization of New Physics at the LHC, Phys. Rev. D 79 (2009) 075020 [arXiv: 0810.3921] [InSPIRE].

[27] LHC New Physics Working Group, Simplified Models for LHC New Physics Searches, J. Phys. G 39 (2012) 105005 [arXiv:1105.2838] [INSPIRE].

[28] LHC Higgs Cross Section Working Group, Handbook of LHC Higgs Cross Sections: 4. Deciphering the Nature of the Higgs Sector, in CERN Yellow Reports: Monographs 2, CERN, Geneva Switzerland (2017) [CERN-2017-002-M] [arXiv: 1610.07922] [INSPIRE]. 
[29] CMS collaboration, Search for supersymmetry using Higgs boson to diphoton decays at $\sqrt{s}=13 \mathrm{TeV}$, JHEP 11 (2019) 109 [arXiv:1908.08500] [INSPIRE].

[30] ATLAS collaboration, Search for direct production of electroweakinos in final states with one lepton, missing transverse momentum and a Higgs boson decaying into two b-jets in pp collisions at $\sqrt{s}=13$ TeV with the ATLAS detector, Eur. Phys. J. C 80 (2020) 691 [arXiv:1909.09226] [INSPIRE].

[31] CMS collaboration, Combined search for electroweak production of charginos and neutralinos in proton-proton collisions at $\sqrt{s}=13 \mathrm{TeV}$, JHEP 03 (2018) 160 [arXiv: 1801.03957] [INSPIRE].

[32] ATLAS collaboration, Search for chargino and neutralino production in final states with a Higgs boson and missing transverse momentum at $\sqrt{s}=13$ TeV with the ATLAS detector, Phys. Rev. D 100 (2019) 012006 [arXiv:1812.09432] [INSPIRE].

[33] M. Dine and W. Fischler, A Phenomenological Model of Particle Physics Based on Supersymmetry, Phys. Lett. B 110 (1982) 227 [inSPIRE].

[34] L. Álvarez-Gaumé, M. Claudson and M.B. Wise, Low-Energy Supersymmetry, Nucl. Phys. B 207 (1982) 96 [inSPIRE].

[35] C.R. Nappi and B.A. Ovrut, Supersymmetric Extension of the $\mathrm{SU}(3) \times \mathrm{SU}(2) \times \mathrm{U}(1)$ Model, Phys. Lett. B 113 (1982) 175 [INSPIRE].

[36] CMS collaboration, Searches for electroweak neutralino and chargino production in channels with Higgs, $Z$, and $W$ bosons in pp collisions at 8 TeV, Phys. Rev. D 90 (2014) 092007 [arXiv: 1409.3168] [INSPIRE].

[37] K.T. Matchev and S.D. Thomas, Higgs and $Z$ boson signatures of supersymmetry, Phys. Rev. D 62 (2000) 077702 [hep-ph/9908482] [INSPIRE].

[38] J.T. Ruderman and D. Shih, General Neutralino NLSPs at the Early LHC, JHEP 08 (2012) 159 [arXiv:1103.6083] [INSPIRE].

[39] ATLAS collaboration, The ATLAS Experiment at the CERN Large Hadron Collider, 2008 JINST 3 S08003 [INSPIRE].

[40] ATLAS collaboration, ATLAS Insertable B-Layer Technical Design Report, CERN-LHCC-2010-013 (2010) [ATLAS-TDR-19] [Addendum ATLAS-TDR-19-ADD-1 (2012)] [INSPIRE].

[41] ATLAS IBL collaboration, Production and Integration of the ATLAS Insertable B-Layer, 2018 JINST 13 T05008 [arXiv:1803.00844] [INSPIRE].

[42] ATLAS collaboration, Performance of the ATLAS Trigger System in 2015, Eur. Phys. J. $C 77$ (2017) 317 [arXiv:1611.09661] [InSPIRE].

[43] ATLAS collaboration, Performance of electron and photon triggers in ATLAS during LHC Run 2, Eur. Phys. J. C 80 (2020) 47 [arXiv: 1909.00761] [InSPIRE].

[44] J. Alwall et al., The automated computation of tree-level and next-to-leading order differential cross sections, and their matching to parton shower simulations, JHEP 07 (2014) 079 [arXiv: 1405.0301] [INSPIRE].

[45] NNPDF collaboration, Parton distributions for the LHC Run II, JHEP 04 (2015) 040 [arXiv:1410.8849] [INSPIRE].

[46] T. Sjöstrand, S. Mrenna and P.Z. Skands, A Brief Introduction to PYTHIA 8.1, Comput. Phys. Commun. 178 (2008) 852 [arXiv:0710.3820] [INSPIRE]. 
[47] ATLAS collaboration, ATLAS PYTHIA 8 tunes to 7 TeV datas, ATL-PHYS-PUB-2014-021 (2014) [INSPIRE].

[48] NNPDF collaboration, Parton distributions with LHC data, Nucl. Phys. B 867 (2013) 244 [arXiv: 1207.1303$]$ [INSPIRE].

[49] J. Debove, B. Fuks and M. Klasen, Threshold resummation for gaugino pair production at hadron colliders, Nucl. Phys. B $\mathbf{8 4 2}$ (2011) 51 [arXiv:1005.2909] [InSPIRE].

[50] B. Fuks, M. Klasen, D.R. Lamprea and M. Rothering, Gaugino production in proton-proton collisions at a center-of-mass energy of 8 TeV, JHEP 10 (2012) 081 [arXiv:1207.2159] [INSPIRE].

[51] B. Fuks, M. Klasen, D.R. Lamprea and M. Rothering, Precision predictions for electroweak superpartner production at hadron colliders with Resummino, Eur. Phys. J. C 73 (2013) 2480 [arXiv: 1304.0790] [INSPIRE].

[52] J. Fiaschi and M. Klasen, Neutralino-chargino pair production at NLO+NLL with resummation-improved parton density functions for LHC Run II, Phys. Rev. D 98 (2018) 055014 [arXiv: 1805.11322] [INSPIRE].

[53] W. Beenakker, M. Klasen, M. Krämer, T. Plehn, M. Spira and P.M. Zerwas, The Production of charginos/neutralinos and sleptons at hadron colliders, Phys. Rev. Lett. 83 (1999) 3780 [Erratum ibid. 100 (2008) 029901] [hep-ph/9906298] [INSPIRE].

[54] C. Borschensky et al., Squark and gluino production cross sections in pp collisions at $\sqrt{s}=13,14,33$ and 100 TeV, Eur. Phys. J. C 74 (2014) 3174 [arXiv:1407.5066] [INSPIRE].

[55] P. Nason, A New method for combining NLO QCD with shower Monte Carlo algorithms, JHEP 11 (2004) 040 [hep-ph/0409146] [INSPIRE].

[56] S. Alioli, P. Nason, C. Oleari and E. Re, NLO Higgs boson production via gluon fusion matched with shower in POWHEG, JHEP 04 (2009) 002 [arXiv:0812.0578] [INSPIRE].

[57] S. Frixione, P. Nason and C. Oleari, Matching NLO QCD computations with Parton Shower simulations: the POWHEG method, JHEP 11 (2007) 070 [arXiv: 0709. 2092] [INSPIRE].

[58] P. Nason and C. Oleari, NLO Higgs boson production via vector-boson fusion matched with shower in POWHEG, JHEP 02 (2010) 037 [arXiv: 0911.5299] [INSPIRE].

[59] S. Alioli, P. Nason, C. Oleari and E. Re, A general framework for implementing NLO calculations in shower Monte Carlo programs: the POWHEG BOX, JHEP 06 (2010) 043 [arXiv: 1002 .2581] [INSPIRE].

[60] ATLAS collaboration, Measurement of the $Z / \gamma^{*}$ boson transverse momentum distribution in pp collisions at $\sqrt{s}=7 \mathrm{TeV}$ with the ATLAS detector, JHEP 09 (2014) 145 [arXiv:1406.3660] [INSPIRE].

[61] H.-L. Lai et al., New parton distributions for collider physics, Phys. Rev. D 82 (2010) 074024 [arXiv: 1007.2241] [INSPIRE].

[62] T. Gleisberg et al., Event generation with SHERPA 1.1, JHEP 02 (2009) 007 [arXiv: 0811.4622] [INSPIRE].

[63] S. Schumann and F. Krauss, A Parton shower algorithm based on Catani-Seymour dipole factorisation, JHEP 03 (2008) 038 [arXiv: 0709.1027] [INSPIRE].

[64] S. Höche, F. Krauss, S. Schumann and F. Siegert, QCD matrix elements and truncated showers, JHEP 05 (2009) 053 [arXiv:0903.1219] [INSPIRE]. 
[65] M. Ciccolini, A. Denner and S. Dittmaier, Strong and electroweak corrections to the production of Higgs +2 jets via weak interactions at the LHC, Phys. Rev. Lett. 99 (2007) 161803 [arXiv: 0707.0381] [INSPIRE].

[66] M. Ciccolini, A. Denner and S. Dittmaier, Electroweak and QCD corrections to Higgs production via vector-boson fusion at the LHC, Phys. Rev. D 77 (2008) 013002 [arXiv:0710.4749] [INSPIRE].

[67] P. Bolzoni, F. Maltoni, S.-O. Moch and M. Zaro, Higgs production via vector-boson fusion at NNLO in QCD, Phys. Rev. Lett. 105 (2010) 011801 [arXiv:1003.4451] [INSPIRE].

[68] K. Mimasu, V. Sanz and C. Williams, Higher Order QCD predictions for Associated Higgs production with anomalous couplings to gauge bosons, JHEP 08 (2016) 039 [arXiv: 1512.02572] [INSPIRE].

[69] O. Brein, A. Djouadi and R. Harlander, NNLO QCD corrections to the Higgs-strahlung processes at hadron colliders, Phys. Lett. B 579 (2004) 149 [hep-ph/0307206] [INSPIRE].

[70] L. Altenkamp, S. Dittmaier, R.V. Harlander, H. Rzehak and T.J.E. Zirke, Gluon-induced Higgs-strahlung at next-to-leading order QCD, JHEP 02 (2013) 078 [arXiv:1211.5015] [INSPIRE].

[71] A. Denner, S. Dittmaier, S. Kallweit and A. Muck, Electroweak corrections to Higgs-strahlung off $W / Z$ bosons at the Tevatron and the LHC with HAWK, JHEP 03 (2012) 075 [arXiv: 1112.5142] [inSPIRE].

[72] C. Anastasiou, C. Duhr, F. Dulat, F. Herzog and B. Mistlberger, Higgs Boson Gluon-Fusion Production in QCD at Three Loops, Phys. Rev. Lett. 114 (2015) 212001 [arXiv: 1503.06056] [INSPIRE].

[73] C. Anastasiou et al., High precision determination of the gluon fusion Higgs boson cross-section at the LHC, JHEP 05 (2016) 058 [arXiv: 1602.00695] [INSPIRE].

[74] S. Actis, G. Passarino, C. Sturm and S. Uccirati, NLO Electroweak Corrections to Higgs Boson Production at Hadron Colliders, Phys. Lett. B 670 (2008) 12 [arXiv:0809.1301] [INSPIRE].

[75] C. Anastasiou, R. Boughezal and F. Petriello, Mixed QCD-electroweak corrections to Higgs boson production in gluon fusion, JHEP 04 (2009) 003 [arXiv: 0811.3458] [INSPIRE].

[76] W. Beenakker, S. Dittmaier, M. Krämer, B. Plumper, M. Spira and P.M. Zerwas, NLO QCD corrections to $t \bar{t} H$ production in hadron collisions, Nucl. Phys. B 653 (2003) 151 [hep-ph/0211352] [INSPIRE].

[77] S. Dawson, C.B. Jackson, L.H. Orr, L. Reina and D. Wackeroth, Associated Higgs production with top quarks at the large hadron collider: NLO QCD corrections, Phys. Rev. D 68 (2003) 034022 [hep-ph/0305087] [INSPIRE].

[78] Y. Zhang, W.-G. Ma, R.-Y. Zhang, C. Chen and L. Guo, QCD NLO and EW NLO

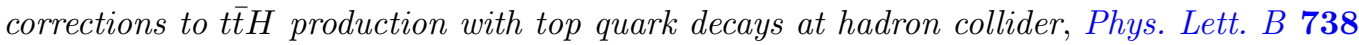
(2014) 1 [arXiv:1407.1110] [INSPIRE].

[79] S. Frixione, V. Hirschi, D. Pagani, H.S. Shao and M. Zaro, Electroweak and QCD corrections to top-pair hadroproduction in association with heavy bosons, JHEP 06 (2015) 184 [arXiv: 1504.03446] [INSPIRE].

[80] S. Dawson, C.B. Jackson, L. Reina and D. Wackeroth, Exclusive Higgs boson production with bottom quarks at hadron colliders, Phys. Rev. D 69 (2004) 074027 [hep-ph/0311067] [INSPIRE]. 
[81] S. Dittmaier, M. Krämer and M. Spira, Higgs radiation off bottom quarks at the Tevatron and the CERN LHC, Phys. Rev. D 70 (2004) 074010 [hep-ph/0309204] [InSPIRE].

[82] R. Harlander, M. Krämer and M. Schumacher, Bottom-quark associated Higgs-boson production: reconciling the four- and five-flavour scheme approach, arXiv:1112.3478 [INSPIRE].

[83] A.D. Martin, W.J. Stirling, R.S. Thorne and G. Watt, Parton distributions for the LHC, Eur. Phys. J. C 63 (2009) 189 [arXiv:0901.0002] [INSPIRE].

[84] ATLAS collaboration, The PYTHIA 8 A3 tune description of ATLAS minimum bias and inelastic measurements incorporating the Donnachie-Landshoff diffractive model, ATL-PHYS-PUB-2016-017 (2016) [INSPIRE].

[85] ATLAS collaboration, The ATLAS Simulation Infrastructure, Eur. Phys. J. C 70 (2010) 823 [arXiv: 1005.4568] [INSPIRE].

[86] GEANT4 collaboration, GEANT4 - a simulation toolkit, Nucl. Instrum. Meth. A 506 (2003) 250 [INSPIRE].

[87] ATLAS collaboration, Electron and photon performance measurements with the ATLAS detector using the 2015-2017 LHC proton-proton collision data, 2019 JINST 14 P12006 [arXiv: 1908.00005] [INSPIRE].

[88] ATLAS collaboration, Topological cell clustering in the ATLAS calorimeters and its performance in LHC Run 1, Eur. Phys. J. C 77 (2017) 490 [arXiv: 1603. 02934] [INSPIRE].

[89] ATLAS collaboration, Measurement of the inclusive isolated prompt photon cross section in pp collisions at $\sqrt{s}=7 \mathrm{TeV}$ with the ATLAS detector, Phys. Rev. D 83 (2011) 052005 [arXiv: 1012.4389] [INSPIRE].

[90] M. Cacciari, G.P. Salam and G. Soyez, The Catchment Area of Jets, JHEP 04 (2008) 005 [arXiv:0802.1188] [INSPIRE].

[91] M. Cacciari, G.P. Salam and S. Sapeta, On the characterisation of the underlying event, JHEP 04 (2010) 065 [arXiv:0912.4926] [INSPIRE].

[92] ATLAS collaboration, Measurements of Higgs boson properties in the diphoton decay channel with $36 \mathrm{fb}^{-1}$ of pp collision data at $\sqrt{\mathrm{s}}=13 \mathrm{TeV}$ with the ATLAS detector, Phys. Rev. D 98 (2018) 052005 [arXiv: 1802.04146] [INSPIRE].

[93] ATLAS collaboration, Measurements of fiducial and differential cross sections for Higgs boson production in the diphoton decay channel at $\sqrt{s}=8$ TeV with ATLAS, JHEP 09 (2014) 112 [arXiv: 1407.4222] [INSPIRE].

[94] ATLAS collaboration, Muon reconstruction performance of the ATLAS detector in proton-proton collision data at $\sqrt{s}=13$ TeV, Eur. Phys. J. C 76 (2016) 292 [arXiv: 1603. 05598] [INSPIRE].

[95] M. Cacciari, G.P. Salam and G. Soyez, The anti- $k_{t}$ jet clustering algorithm, JHEP 04 (2008) 063 [arXiv: 0802.1189] [INSPIRE].

[96] M. Cacciari, G.P. Salam and G. Soyez, FastJet User Manual, Eur. Phys. J. C 72 (2012) 1896 [arXiv:1111.6097] [INSPIRE].

[97] ATLAS collaboration, Tagging and suppression of pileup jets, ATL-PHYS-PUB-2014-001 (2014) [INSPIRE].

[98] ATLAS collaboration, Selection of jets produced in $13 \mathrm{TeV}$ proton-proton collisions with the ATLAS detector, ATLAS-CONF-2015-029 (2015) [INSPIRE]. 
[99] ATLAS collaboration, ATLAS b-jet identification performance and efficiency measurement with $t \bar{t}$ events in pp collisions at $\sqrt{s}=13$ TeV, Eur. Phys. J. C 79 (2019) 970 [arXiv: 1907.05120] [INSPIRE].

[100] ATLAS collaboration, Optimisation and performance studies of the ATLAS b-tagging algorithms for the 2017-18 LHC run, ATL-PHYS-PUB-2017-013 (2017) [INSPIRE].

[101] ATLAS collaboration, Performance of missing transverse momentum reconstruction with the ATLAS detector using proton-proton collisions at $\sqrt{s}=13$ TeV, Eur. Phys. J. C 78 (2018) 903 [arXiv: 1802.08168] [INSPIRE].

[102] ATLAS collaboration, Measurement of isolated-photon pair production in pp collisions at $\sqrt{s}=7 \mathrm{TeV}$ with the ATLAS detector, JHEP 01 (2013) 086 [arXiv:1211.1913] [INSPIRE].

[103] ATLAS collaboration, Search for Scalar Diphoton Resonances in the Mass Range 65-600 GeV with the ATLAS Detector in pp Collision Data at $\sqrt{s}=8 \mathrm{TeV}$, Phys. Rev. Lett. 113 (2014) 171801 [arXiv: 1407.6583] [INSPIRE].

[104] ATLAS collaboration, Measurement of Higgs boson production in the diphoton decay channel in pp collisions at center-of-mass energies of 7 and $8 \mathrm{TeV}$ with the ATLAS detector, Phys. Rev. D 90 (2014) 112015 [arXiv:1408.7084] [INSPIRE].

[105] CDF collaboration, Search for new particles decaying into dijets in proton-antiproton collisions at $\sqrt{s}=1.96 \mathrm{TeV}$, Phys. Rev. D 79 (2009) 112002 [arXiv:0812.4036] [INSPIRE].

[106] ATLAS collaboration, Luminosity determination in pp collisions at $\sqrt{s}=13 \mathrm{TeV}$ using the ATLAS detector at the LHC, ATLAS-CONF-2019-021 (2019) [INSPIRE].

[107] G. Avoni et al., The new LUCID-2 detector for luminosity measurement and monitoring in ATLAS, 2018 JINST 13 P07017 [INSPIRE].

[108] ATLAS collaboration, Search for resonances in diphoton events at $\sqrt{s}=13 \mathrm{TeV}$ with the ATLAS detector, JHEP 09 (2016) 001 [arXiv: 1606.03833] [INSPIRE].

[109] ATLAS collaboration, Jet energy scale measurements and their systematic uncertainties in proton-proton collisions at $\sqrt{s}=13 \mathrm{TeV}$ with the ATLAS detector, Phys. Rev. D 96 (2017) 072002 [arXiv: 1703.09665] [INSPIRE].

[110] S. Dulat et al., New parton distribution functions from a global analysis of quantum chromodynamics, Phys. Rev. D 93 (2016) 033006 [arXiv: 1506.07443] [InSPIRE].

[111] L.A. Harland-Lang, A.D. Martin, P. Motylinski and R.S. Thorne, Parton distributions in the LHC era: MMHT 2014 PDFs, Eur. Phys. J. C 75 (2015) 204 [arXiv:1412.3989] [INSPIRE].

[112] A.L. Read, Presentation of search results: The $C L_{s}$ technique, J. Phys. G 28 (2002) 2693 [INSPIRE].

[113] G. Cowan, K. Cranmer, E. Gross and O. Vitells, Asymptotic formulae for likelihood-based tests of new physics, Eur. Phys. J. C 71 (2011) 1554 [Erratum ibid. 73 (2013) 2501] [arXiv: 1007.1727] [INSPIRE].

[114] ATLAS collaboration, ATLAS Computing Acknowledgements, ATL-GEN-PUB-2016-002 (2016). 


\section{The ATLAS collaboration}

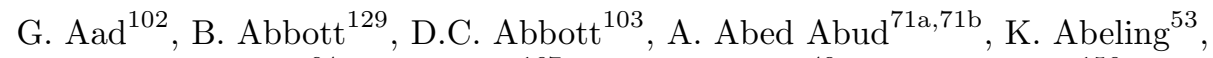
D.K. Abhayasinghe ${ }^{94}$, S.H. Abidi ${ }^{167}$, O.S. AbouZeid ${ }^{40}$, N.L. Abraham ${ }^{156}$, H. Abramowicz ${ }^{161}$, H. Abreu ${ }^{160}$, Y. Abulaiti ${ }^{6}$, B.S. Acharya ${ }^{67 a, 67 b, o}$, B. Achkar ${ }^{53}$, S. Adachi ${ }^{163}$, L. Adam ${ }^{100}$, C. Adam Bourdarios ${ }^{5}$, L. Adamczyk ${ }^{84 a}$, L. Adamek ${ }^{167}$, J. Adelman ${ }^{121}$, M. Adersberger ${ }^{114}$, A. Adiguzel ${ }^{12 \mathrm{c}, a \mathrm{k}}$, S. Adorni ${ }^{54}$, T. Adye ${ }^{144}$, A.A. Affolder ${ }^{146}$, Y. Afik ${ }^{160}$, C. Agapopoulou ${ }^{65}$, M.N. Agaras ${ }^{38}$, A. Aggarwal ${ }^{119}$, C. Agheorghiesei ${ }^{27 c}$, J.A. Aguilar-Saavedra ${ }^{140 f, 140 a, a j}$, F. Ahmadov ${ }^{80}$, W.S. Ahmed ${ }^{104}$, X. Ai ${ }^{18}$, G. Aielli ${ }^{74 a, 74 b}$, S. Akatsuka ${ }^{86}$, T.P.A. Åkesson ${ }^{97}$, E. Akilli ${ }^{54}$, A.V. Akimov ${ }^{111}$, K. Al Khoury ${ }^{65}$, G.L. Alberghi ${ }^{23 b, 23 a}$, J. Albert ${ }^{176}$,

M.J. Alconada Verzini ${ }^{161}$, S. Alderweireldt ${ }^{36}$, M. Aleksa ${ }^{36}$, I.N. Aleksandrov ${ }^{80}$, C. Alexa ${ }^{27 b}$, D. Alexandre ${ }^{19}$, T. Alexopoulos ${ }^{10}$, A. Alfonsi ${ }^{120}$, F. Alfonsi ${ }^{23 b, 23 a}$, M. Alhroob ${ }^{129}$, B. Ali ${ }^{142}$, G. Alimonti ${ }^{69 a}$, J. Alison ${ }^{37}$, S.P. Alkire ${ }^{148}$, C. Allaire ${ }^{65}$, B.M.M. Allbrooke ${ }^{156}$, B.W. Allen ${ }^{132}$, P.P. Allport ${ }^{21}$, A. Aloisio ${ }^{70 a, 70 b}$, A. Alonso ${ }^{40}$, F. Alonso ${ }^{89}$, C. Alpigiani ${ }^{148}$, A.A. Alshehri ${ }^{57}$, M. Alvarez Estevez ${ }^{99}$, D. Álvarez Piqueras ${ }^{174}$, M.G. Alviggi ${ }^{70 a, 70 b}$, Y. Amaral Coutinho ${ }^{81 b}$, A. Ambler ${ }^{104}$, L. Ambroz ${ }^{135}$, C. Amelung ${ }^{26}$, D. Amidei ${ }^{106}$, S.P. Amor Dos Santos ${ }^{140 a}$, S. Amoroso ${ }^{46}$, C.S. Amrouche ${ }^{54}$, F. An ${ }^{79}$, C. Anastopoulos ${ }^{149}$, N. Andari ${ }^{145}$, T. Andeen ${ }^{11}$, C.F. Anders ${ }^{61 b}$, J.K. Anders ${ }^{20}$, A. Andreazza ${ }^{69 a, 69 b}$, V. Andrei ${ }^{61 a}$, C.R. Anelli ${ }^{176}$, S. Angelidakis ${ }^{38}$, A. Angerami ${ }^{39}$, A.V. Anisenkov ${ }^{122 b, 122 a}$, A. Annovi ${ }^{72 a}$, C. Antel ${ }^{61 a}$, M.T. Anthony ${ }^{149}$, M. Antonelli ${ }^{51}$, D.J.A. Antrim ${ }^{171}$, F. Anulli ${ }^{73 a}$, M. Aoki ${ }^{82}$,

J.A. Aparisi Pozo ${ }^{174}$, L. Aperio Bella ${ }^{15 a}$, G. Arabidze ${ }^{107}$, J.P. Araque ${ }^{140 a}$, V. Araujo Ferraz ${ }^{81 b}$, R. Araujo Pereira ${ }^{81 b}$, C. Arcangeletti ${ }^{51}$, A.T.H. Arce ${ }^{49}$, F.A. Arduh ${ }^{89}$, J-F. Arguin ${ }^{110}$, S. Argyropoulos ${ }^{78}$, J.-H. Arling ${ }^{46}$, A.J. Armbruster ${ }^{36}$, A. Armstrong ${ }^{171}$, O. Arnaez ${ }^{167}$, H. Arnold ${ }^{120}$, Z.P. Arrubarrena Tame ${ }^{114}$, A. Artamonov ${ }^{124, *}$, G. Artoni ${ }^{135}$, S. Artz ${ }^{100}$, S. Asai ${ }^{163}$, N. Asbah ${ }^{59}$, E.M. Asimakopoulou ${ }^{172}$, L. Asquith ${ }^{156}$, J. Assahsah ${ }^{35 d}$, K. Assamagan ${ }^{29}$, R. Astalos ${ }^{28 a}$, R.J. Atkin ${ }^{33 a}$, M. Atkinson ${ }^{173}$, N.B. Atlay ${ }^{19}$, H. Atmani ${ }^{65}$, K. Augsten ${ }^{142}$, G. Avolio ${ }^{36}$, R. Avramidou ${ }^{60 a}$, M.K. Ayoub ${ }^{15 a}$, A.M. Azoulay ${ }^{168 b}$, G. Azuelos ${ }^{110, a x}$, H. Bachacou ${ }^{145}$, K. Bachas ${ }^{68 a, 68 b}$, M. Backes ${ }^{135}$, F. Backman ${ }^{45 a, 45 b}$, P. Bagnaia ${ }^{73 a, 73 b}$, M. Bahmani ${ }^{85}$, H. Bahrasemani ${ }^{152}$, A.J. Bailey ${ }^{174}$, V.R. Bailey ${ }^{173}$, J.T. Baines ${ }^{144}$, M. Bajic ${ }^{40}$, C. Bakalis $^{10}$, O.K. Baker ${ }^{183}$, P.J. Bakker ${ }^{120}$, D. Bakshi Gupta ${ }^{8}$, S. Balaji ${ }^{157}$, E.M. Baldin ${ }^{122 b, 122 a}$, P. Balek ${ }^{180}$, F. Balli ${ }^{145}$, W.K. Balunas ${ }^{135}$, J. Balz ${ }^{100}$, E. Banas ${ }^{85}$, A. Bandyopadhyay ${ }^{24}$, Sw. Banerjee ${ }^{181, j}$, A.A.E. Bannoura ${ }^{182}$, L. Barak ${ }^{161}$, W.M. Barbe ${ }^{38}$, E.L. Barberio ${ }^{105}$, D. Barberis ${ }^{55 b, 55 a}$, M. Barbero ${ }^{102}$, G. Barbour ${ }^{95}$, T. Barillari ${ }^{115}$, M-S. Barisits ${ }^{36}$, J. Barkeloo ${ }^{132}$, T. Barklow ${ }^{153}$, R. Barnea ${ }^{160}$, S.L. Barnes ${ }^{60 c}$, B.M. Barnett ${ }^{144}$, R.M. Barnett ${ }^{18}$, Z. Barnovska-Blenessy ${ }^{60 a}$, A. Baroncelli ${ }^{60 a}$, G. Barone $^{29}$, A.J. Barr ${ }^{135}$,

L. Barranco Navarro ${ }^{45 a, 45 b}$, F. Barreiro ${ }^{99}$, J. Barreiro Guimarães da Costa ${ }^{15 a}$, S. Barsov ${ }^{138}$, R. Bartoldus ${ }^{153}$, G. Bartolini ${ }^{102}$, A.E. Barton ${ }^{90}$, P. Bartos ${ }^{28 a}$, A. Basalaev ${ }^{46}$, A. Bassalat ${ }^{65, \text { ar }}$, M.J. Basso ${ }^{167}$, R.L. Bates ${ }^{57}$, S. Batlamous ${ }^{35 e}$, J.R. Batley ${ }^{32}$, B. Batool ${ }^{151}$, M. Battaglia ${ }^{146}$, M. Bauce ${ }^{73 a, 73 b}$, F. Bauer ${ }^{145}$, K.T. Bauer ${ }^{171}$, H.S. Bawa ${ }^{31, m}$, J.B. Beacham ${ }^{49}$, T. Beau ${ }^{136}$, P.H. Beauchemin ${ }^{170}$, F. Becherer ${ }^{52}$, P. Bechtle ${ }^{24}$, H.C. Beck ${ }^{53}$, H.P. Beck ${ }^{20, s}$, K. Becker ${ }^{52}$, M. Becker ${ }^{100}$, C. Becot ${ }^{46}$, A. Beddall ${ }^{12 d}$, A.J. Beddall ${ }^{12 \mathrm{a}}$, V.A. Bednyakov ${ }^{80}$, M. Bedognetti ${ }^{120}$, C.P. Bee ${ }^{155}$, T.A. Beermann ${ }^{77}$, M. Begalli ${ }^{81 b}$, M. Begel $^{29}$, A. Behera ${ }^{155}$, J.K. Behr ${ }^{46}$, F. Beisiegel ${ }^{24}$, A.S. Bell ${ }^{95}$, G. Bella ${ }^{161}$, L. Bellagamba ${ }^{23 b}$, A. Bellerive ${ }^{34}$, P. Bellos ${ }^{9}$, K. Beloborodov ${ }^{122 b, 122 a}$, K. Belotskiy ${ }^{112}$, N.L. Belyaev ${ }^{112}$, D. Benchekroun ${ }^{35 a}$, N. Benekos ${ }^{10}$, Y. Benhammou ${ }^{161}$, D.P. Benjamin ${ }^{6}$, M. Benoit ${ }^{54}$, J.R. Bensinger ${ }^{26}$, S. Bentvelsen ${ }^{120}$, L. Beresford $^{135}$, M. Beretta ${ }^{51}$, D. Berge ${ }^{46}$, E. Bergeaas Kuutmann ${ }^{172}$, N. Berger ${ }^{5}$, B. Bergmann ${ }^{142}$, L.J. Bergsten ${ }^{26}$, J. Beringer ${ }^{18}$, S. Berlendis ${ }^{7}$, N.R. Bernard ${ }^{103}$, G. Bernardi ${ }^{136}$, C. Bernius ${ }^{153}$, F.U. Bernlochner ${ }^{24}$, T. Berry ${ }^{94}$, P. Berta ${ }^{100}$, C. Bertella ${ }^{15 a}$, I.A. Bertram ${ }^{90}$, 
O. Bessidskaia Bylund ${ }^{182}$, N. Besson ${ }^{145}$, A. Bethani ${ }^{101}$, S. Bethke ${ }^{115}$, A. Betti ${ }^{24}$, A.J. Bevan ${ }^{93}$, J. Beyer ${ }^{115}$, D.S. Bhattacharya ${ }^{177}$, R. Bi ${ }^{139}$, R.M. Bianchi ${ }^{139}$, O. Biebel ${ }^{114}$, D. Biedermann ${ }^{19}$, R. Bielski ${ }^{36}$, K. Bierwagen ${ }^{100}$, N.V. Biesuz ${ }^{72 a, 72 b}$, M. Biglietti ${ }^{75 a}$, T.R.V. Billoud ${ }^{110}$, M. Bindi $^{53}$,

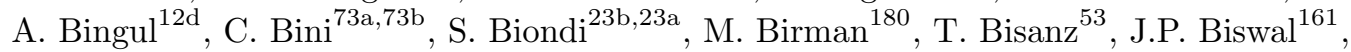
D. Biswas ${ }^{181, j}$, A. Bitadze ${ }^{101}$, C. Bittrich ${ }^{48}$, K. Bjørke ${ }^{134}$, K.M. Black ${ }^{25}$, T. Blazek ${ }^{28 a}$, I. Bloch ${ }^{46}$, C. Blocker ${ }^{26}$, A. Blue ${ }^{57}$, U. Blumenschein ${ }^{93}$, G.J. Bobbink ${ }^{120}$, V.S. Bobrovnikov ${ }^{122 b, 122 a}$, S.S. Bocchetta ${ }^{97}$, A. Bocci ${ }^{49}$, D. Boerner ${ }^{46}$, D. Bogavac ${ }^{14}$, A.G. Bogdanchikov ${ }^{122 b, 122 a}$, C. Bohm ${ }^{45 a}$, V. Boisvert ${ }^{94}$, P. Bokan ${ }^{53,172,53}$, T. Bold ${ }^{84 a}$, A.S. Boldyrev ${ }^{113}$, A.E. Bolz ${ }^{61 b}$, M. Bomben ${ }^{136}$, M. Bona ${ }^{93}$, J.S. Bonilla ${ }^{132}$, M. Boonekamp ${ }^{145}$, H.M. Borecka-Bielska ${ }^{91}$, A. Borisov ${ }^{123}$, G. Borissov ${ }^{90}$, J. Bortfeldt ${ }^{36}$, D. Bortoletto ${ }^{135}$, D. Boscherini ${ }^{23 b}$, M. Bosman ${ }^{14}$, J.D. Bossio Sola ${ }^{104}$, K. Bouaouda ${ }^{35 a}$, J. Boudreau ${ }^{139}$, E.V. Bouhova-Thacker ${ }^{90}$, D. Boumediene ${ }^{38}$, S.K. Boutle ${ }^{57}$, A. Boveia ${ }^{127}$, J. Boyd ${ }^{36}$, D. Boye ${ }^{33 c, \text { as }}$, I.R. Boyko ${ }^{80}$, A.J. Bozson ${ }^{94}$, J. Bracinik ${ }^{21}$, N. Brahimi ${ }^{102}$, G. Brandt ${ }^{182}$, O. Brandt ${ }^{32}$, F. Braren ${ }^{46}$, B. Brau ${ }^{103}$, J.E. Brau ${ }^{132}$, W.D. Breaden Madden ${ }^{57}$, K. Brendlinger ${ }^{46}$, L. Brenner ${ }^{46}$, R. Brenner ${ }^{172}$, S. Bressler ${ }^{180}$, B. Brickwedde ${ }^{100}$, D.L. Briglin ${ }^{21}$, D. Britton ${ }^{57}$, D. Britzger ${ }^{115}$, I. Brock ${ }^{24}$, R. Brock ${ }^{107}$, G. Brooijmans ${ }^{39}$, W.K. Brooks ${ }^{147}$, E. Brost ${ }^{121}$, J.H Broughton ${ }^{21}$, P.A. Bruckman de Renstrom ${ }^{85}$, D. Bruncko ${ }^{28 b}$, A. Bruni ${ }^{23 b}$, G. Bruni ${ }^{23 b}$, L.S. Bruni ${ }^{120}$, S. Bruno ${ }^{74 a, 74 b}$, B.H. Brunt ${ }^{32}$, M. Bruschi ${ }^{23 b}$, N. Bruscino ${ }^{139}$, P. Bryant ${ }^{37}$, L. Bryngemark ${ }^{97}$, T. Buanes ${ }^{17}$, Q. Buat ${ }^{36}$, P. Buchholz ${ }^{151}$, A.G. Buckley ${ }^{57}$, I.A. Budagov ${ }^{80}$, M.K. Bugge ${ }^{134}$, F. Bührer ${ }^{52}$, O. Bulekov ${ }^{112}$, T.J. Burch ${ }^{121}$, S. Burdin ${ }^{91}$, C.D. Burgard ${ }^{120}$, A.M. Burger ${ }^{130}$, B. Burghgrave ${ }^{8}$, J.T.P. Burr ${ }^{46}$, C.D. Burton ${ }^{11}$, J.C. Burzynski ${ }^{103}$, V. Büscher ${ }^{100}$, E. Buschmann ${ }^{53}$, P.J. Bussey ${ }^{57}$, J.M. Butler ${ }^{25}$, C.M. Buttar ${ }^{57}$, J.M. Butterworth ${ }^{95}$, P. Butti ${ }^{36}$, W. Buttinger ${ }^{36}$, A. Buzatu ${ }^{158}$, A.R. Buzykaev ${ }^{122 b, 122 a}$, G. Cabras ${ }^{23 b, 23 a}$, S. Cabrera Urbán ${ }^{174}$, D. Caforio ${ }^{56}$, H. Cai ${ }^{173}$, V.M.M. Cairo ${ }^{153}$, O. Cakir ${ }^{4 a}$, N. Calace $^{36}$, P. Calafiura $^{18}$, A. Calandri ${ }^{102}$, G. Calderini ${ }^{136}$, P. Calfayan ${ }^{66}$, G. Callea ${ }^{57}$, L.P. Caloba ${ }^{81 b}$, S. Calvente Lopez ${ }^{99}$, D. Calvet $^{38}$, S. Calvet ${ }^{38}$, T.P. Calvet ${ }^{155}$, M. Calvetti ${ }^{72 a, 72 b}$, R. Camacho Toro ${ }^{136}$, S. Camarda ${ }^{36}$, D. Camarero Munoz ${ }^{99}$, P. Camarri ${ }^{74 a, 74 b}$, D. Cameron ${ }^{134}$, R. Caminal Armadans ${ }^{103}$, C. Camincher $^{36}$, S. Campana ${ }^{36}$, M. Campanelli ${ }^{95}$, A. Camplani ${ }^{40}$, A. Campoverde ${ }^{151}$, V. Canale ${ }^{70 a, 70 b}$, A. Canesse ${ }^{104}$, M. Cano Bret ${ }^{60 c}$, J. Cantero ${ }^{130}$, T. Cao ${ }^{161}$, Y. Cao ${ }^{173}$, M.D.M. Capeans Garrido ${ }^{36}$,

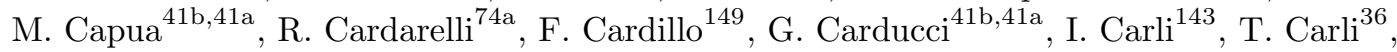
G. Carlino $^{70 a}$, B.T. Carlson ${ }^{139}$, L. Carminati ${ }^{69 a, 69 b}$, R.M.D. Carney ${ }^{45 a, 45 b}$, S. Caron ${ }^{119}$, E. Carquin ${ }^{147 \mathrm{~d}}$, S. Carrá ${ }^{46}$, J.W.S. Carter ${ }^{167}$, M.P. Casado ${ }^{14, \mathrm{e}}$, A.F. Casha ${ }^{167}$, D.W. Casper ${ }^{171}$, R. Castelijn ${ }^{120}$, F.L. Castillo ${ }^{174}$, V. Castillo Gimenez ${ }^{174}$, N.F. Castro ${ }^{140 a, 140 e}{ }^{\text {, A. Catinaccio }}{ }^{36}$, J.R. Catmore ${ }^{134}$, A. Cattai ${ }^{36}$, J. Caudron ${ }^{24}$, V. Cavaliere $^{29}$, E. Cavallaro ${ }^{14}$, M. Cavalli-Sforza ${ }^{14}$ V. Cavasinni ${ }^{72 a, 72 b}$, E. Celebi ${ }^{12 b}$, F. Ceradini ${ }^{75 a, 75 b}$, L. Cerda Alberich $^{174}$, K. Cerny ${ }^{131}$, A.S. Cerqueira ${ }^{81 a}$, A. Cerri ${ }^{156}$, L. Cerrito ${ }^{74 a, 74 b}$, F. Cerutti $^{18}$, A. Cervelli ${ }^{23 b, 23 a}$, S.A. Cetin ${ }^{12 b}$, Z. Chadi ${ }^{35 a}$, D. Chakraborty ${ }^{121}$, S.K. Chan $^{59}$, W.S. Chan ${ }^{120}$, W.Y. Chan ${ }^{91}$, J.D. Chapman ${ }^{32}$, B. Chargeishvili ${ }^{159 b}$, D.G. Charlton ${ }^{21}$, T.P. Charman $^{93}$, C.C. Chau ${ }^{34}$, S. Che ${ }^{127}$, S. Chekanov ${ }^{6}$, S.V. Chekulaev ${ }^{168 a}$, G.A. Chelkov ${ }^{80, a p}$, M.A. Chelstowska ${ }^{36}$, B. Chen ${ }^{79}$, C. Chen ${ }^{60 a}$, C.H. Chen ${ }^{79}$, H. Chen ${ }^{29}$, J. Chen ${ }^{60 a}$, J. Chen ${ }^{39}$, S. Chen ${ }^{137}$, S.J. Chen ${ }^{15 c}$, X. Chen ${ }^{15 b, a w}$, Y. Chen ${ }^{83}$, Y-H. Chen ${ }^{46}$, H.C. Cheng ${ }^{63 a}$, H.J. Cheng ${ }^{15 a}$, A. Cheplakov ${ }^{80}$, E. Cheremushkina ${ }^{123}$, R. Cherkaoui El Moursli ${ }^{35 e}$, E. Cheu ${ }^{7}$, K. Cheung ${ }^{64}$, T.J.A. Chevalérias ${ }^{145}$, L. Chevalier ${ }^{145}$,

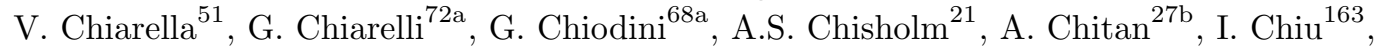
Y.H. Chiu ${ }^{176}$, M.V. Chizhov ${ }^{80}$, K. Choi ${ }^{66}$, A.R. Chomont ${ }^{73 a, 73 b}$, S. Chouridou ${ }^{162}$, Y.S. Chow ${ }^{120}$, M.C. Chu ${ }^{63 a}$, X. Chu ${ }^{15 a, 15 d}$, J. Chudoba ${ }^{141}$, A.J. Chuinard ${ }^{104}$, J.J. Chwastowski ${ }^{85}$, L. Chytka ${ }^{131}$, D. Cieri ${ }^{115}$, K.M. Ciesla ${ }^{85}$, D. Cinca ${ }^{47}$, V. Cindro ${ }^{92}$, I.A. Cioară ${ }^{27 b}$, A. Ciocio ${ }^{18}$, F. Cirotto ${ }^{70 a, 70 b}$, Z.H. Citron ${ }^{180, k}$, M. Citterio ${ }^{69 a}$, D.A. Ciubotaru ${ }^{27 b}$, B.M. Ciungu ${ }^{167}$, A. Clark ${ }^{54}$, M.R. Clark ${ }^{39}$,

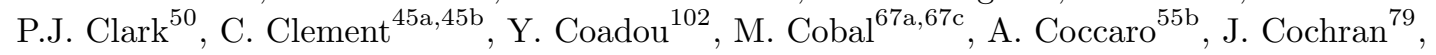


H. Cohen ${ }^{161}$, A.E.C. Coimbra ${ }^{36}$, L. Colasurdo ${ }^{119}$, B. Cole ${ }^{39}$, A.P. Colijn ${ }^{120}$, J. Collot ${ }^{58}$, P. Conde Muiño ${ }^{140 a, f}$, E. Coniavitis ${ }^{52}$, S.H. Connell ${ }^{33 c}$, I.A. Connelly ${ }^{57}$, S. Constantinescu ${ }^{27 b}$, F. Conventi ${ }^{70 a, a y}$, A.M. Cooper-Sarkar ${ }^{135}$, F. Cormier $^{175}$, K.J.R. Cormier $^{167}$, L.D. Corpe ${ }^{95}$,

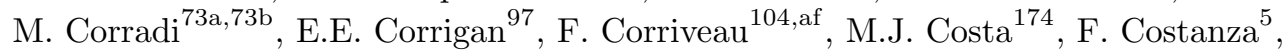
D. Costanzo ${ }^{149}$, G. Cowan $^{94}$, J.W. Cowley ${ }^{32}$, J. Crane ${ }^{101}$, K. Cranmer $^{125}$, S.J. Crawley ${ }^{57}$, R.A. Creager $^{137}$, S. Crépé-Renaudin ${ }^{58}$, F. Crescioli ${ }^{136}$, M. Cristinziani ${ }^{24}$, V. Croft $^{120}$, G. Crosetti ${ }^{41 \mathrm{~b}, 41 \mathrm{a}}$, A. Cueto ${ }^{5}$, T. Cuhadar Donszelmann ${ }^{149}$, A.R. Cukierman ${ }^{153}$, S. Czekierda ${ }^{85}$, P. Czodrowski ${ }^{36}$, M.J. Da Cunha Sargedas De Sousa ${ }^{60 b}$, J.V. Da Fonseca Pinto ${ }^{81 b}$, C. Da Via ${ }^{101}$,

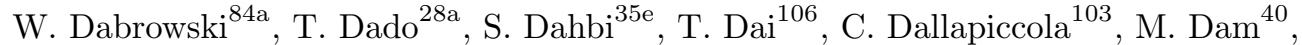
G. D'amen ${ }^{29}$, V. D'Amico ${ }^{75 a, 75 b}$, J. Damp ${ }^{100}$, J.R. Dandoy ${ }^{137}$, M.F. Daneri ${ }^{30}$, N.P. Dang ${ }^{181, j}$, N.S. Dann ${ }^{101}$, M. Danninger ${ }^{175}$, V. Dao ${ }^{36}$, G. Darbo $^{55 b}$, O. Dartsi ${ }^{5}$, A. Dattagupta ${ }^{132}$, T. Daubney ${ }^{46}$, S. D’Auria ${ }^{69 a, 69 b}$, W. Davey ${ }^{24}$, C. David ${ }^{46}$, T. Davidek ${ }^{143}$, D.R. Davis ${ }^{49}$, I. Dawson ${ }^{149}$, K. De ${ }^{8}$, R. De Asmundis ${ }^{70 a}$, M. De Beurs ${ }^{120}$, S. De Castro ${ }^{23 b, 23 a}$, S. De Cecco ${ }^{73 a}, 73 b$, N. De Groot ${ }^{119}$, P. de Jong ${ }^{120}$, H. De la Torre ${ }^{107}$, A. De Maria ${ }^{15 c}$,

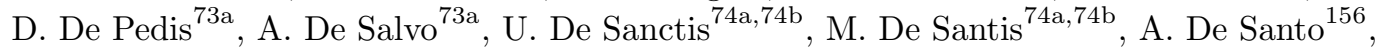
K. De Vasconcelos Corga ${ }^{102}$, J.B. De Vivie De Regie ${ }^{65}$, C. Debenedetti ${ }^{146}$, D.V. Dedovich ${ }^{80}$, A.M. Deiana ${ }^{42}$, M. Del Gaudio ${ }^{41 b, 41 a}$, J. Del Peso ${ }^{99}$, Y. Delabat Diaz ${ }^{46}$, D. Delgove ${ }^{65}$, F. Deliot ${ }^{145, \mathrm{r}}$, C.M. Delitzsch ${ }^{7}$, M. Della Pietra ${ }^{70 a, 70 b}$, D. Della Volpe ${ }^{54}$, A. Dell'Acqua ${ }^{36}$, L. Dell'Asta ${ }^{74 a, 74 b}$, M. Delmastro ${ }^{5}$, C. Delporte ${ }^{65}$, P.A. Delsart ${ }^{58}$, D.A. DeMarco ${ }^{167}$, S. Demers ${ }^{183}$, M. Demichev ${ }^{80}$, G. Demontigny ${ }^{110}$, S.P. Denisov ${ }^{123}$, D. Denysiuk ${ }^{120}$,

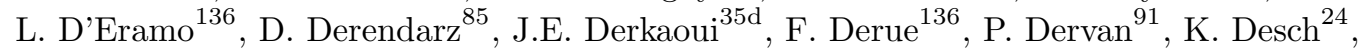
C. Deterre ${ }^{46}$, K. Dette ${ }^{167}$, C. Deutsch ${ }^{24}$, M.R. Devesa ${ }^{30}$, P.O. Deviveiros ${ }^{36}$, A. Dewhurst ${ }^{144}$, F.A. Di Bello ${ }^{54}$, A. Di Ciaccio ${ }^{74 a, 74 b}$, L. Di Ciaccio ${ }^{5}$, W.K. Di Clemente ${ }^{137}$, C. Di Donato ${ }^{70 a, 70 b}$, A. Di Girolamo ${ }^{36}$, G. Di Gregorio ${ }^{72 a, 72 b}$, B. Di Micco ${ }^{75 a, 75 b}$, R. Di Nardo ${ }^{103}$, K.F. Di Petrillo ${ }^{59}$, R. Di Sipio ${ }^{167}$, D. Di Valentino ${ }^{34}$, C. Diaconu ${ }^{102}$, F.A. Dias ${ }^{40}$, T. Dias Do Vale ${ }^{140 a}$, M.A. Diaz $^{147 a}$, J. Dickinson ${ }^{18}$, E.B. Diehl ${ }^{106}$, J. Dietrich ${ }^{19}$, S. Díez Cornell ${ }^{46}$, A. Dimitrievska ${ }^{18}$, W. Ding ${ }^{15 b}$, J. Dingfelder ${ }^{24}$, F. Dittus ${ }^{36}$, F. Djama ${ }^{102}$, T. Djobava ${ }^{159 b}$, J.I. Djuvsland ${ }^{17}$, M.A.B. Do Vale ${ }^{81 c}$, M. Dobre ${ }^{27 b}$, D. Dodsworth ${ }^{26}$, C. Doglioni ${ }^{97}$, J. Dolejsi ${ }^{143}$, Z. Dolezal ${ }^{143}$, M. Donadelli ${ }^{81 d}$, B. Dong ${ }^{60 c}$, J. Donini ${ }^{38}$, A. D’onofrio ${ }^{93}$, M. D'Onofrio ${ }^{91}$, J. Dopke ${ }^{144}$, A. Doria ${ }^{70 a}$, M.T. Dova ${ }^{89}$, A.T. Doyle ${ }^{57}$, E. Drechsler ${ }^{152}$, E. Dreyer ${ }^{152}$, T. Dreyer ${ }^{53}$, A.S. Drobac ${ }^{170}$, Y. Duan ${ }^{60 b}$,

F. Dubinin ${ }^{111}$, M. Dubovsky ${ }^{28 a}$, A. Dubreuil ${ }^{54}$, E. Duchovni ${ }^{180}$, G. Duckeck ${ }^{114}$, A. Ducourthial ${ }^{136}$, O.A. Ducu ${ }^{110}$, D. Duda ${ }^{115}$, A. Dudarev ${ }^{36}$, A.C. Dudder ${ }^{100}$, E.M. Duffield ${ }^{18}$, L. Duflot ${ }^{65}$,

M. Dührssen ${ }^{36}$, C. Dülsen ${ }^{182}$, M. Dumancic ${ }^{180}$, A.E. Dumitriu ${ }^{27 b}$, A.K. Duncan ${ }^{57}$, M. Dunford ${ }^{61 a}$, A. Duperrin ${ }^{102}$, H. Duran Yildiz ${ }^{4 a}$, M. Düren ${ }^{56}$, A. Durglishvili ${ }^{159 b}$, D. Duschinger ${ }^{48}$, B. Dutta ${ }^{46}$, D. Duvnjak ${ }^{1}$, G.I. Dyckes ${ }^{137}$, M. Dyndal ${ }^{36}$, S. Dysch ${ }^{101}$, B.S. Dziedzic ${ }^{85}$, K.M. Ecker ${ }^{115}$, R.C. Edgar $^{106}$, M.G. Eggleston ${ }^{49}$, T. Eifert $^{36}$, G. Eigen $^{17}$, K. Einsweiler ${ }^{18}$, T. Ekelof ${ }^{172}$, H. El Jarrari ${ }^{35 e}$, M. El Kacimi ${ }^{35 c}$, R. El Kosseifi ${ }^{102}$, V. Ellajosyula ${ }^{172}$, M. Ellert ${ }^{172}$, F. Ellinghaus ${ }^{182}$, A.A. Elliot ${ }^{93}$, N. Ellis ${ }^{36}$, J. Elmsheuser ${ }^{29}$, M. Elsing ${ }^{36}$, D. Emeliyanov ${ }^{144}$, A. $\operatorname{Emerman}^{39}$, Y. Enari ${ }^{163}$, M.B. Epland ${ }^{49}$, J. Erdmann ${ }^{47}$, A. Ereditato ${ }^{20}$, M. Errenst ${ }^{36}$, M. Escalier ${ }^{65}$, C. Escobar ${ }^{174}$, O. Estrada Pastor ${ }^{174}$, E. Etzion ${ }^{161}$, H. Evans ${ }^{66}$, A. Ezhilov ${ }^{138}$, F. Fabbri ${ }^{57}$, L. Fabbri ${ }^{23 b, 23 a}$, V. Fabiani ${ }^{119}$, G. Facini ${ }^{95}$, R.M. Faisca Rodrigues Pereira ${ }^{140 a}$, R.M. Fakhrutdinov ${ }^{123}$, S. Falciano ${ }^{73 a}$, P.J. Falke ${ }^{5}$, S. Falke ${ }^{5}$, J. Faltova ${ }^{143}$, Y. Fang ${ }^{15 a}$,

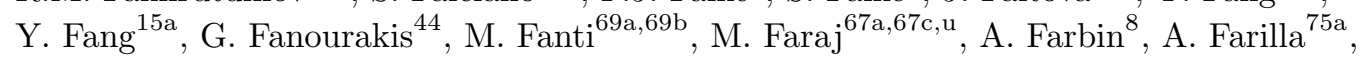
E.M. Farina ${ }^{71 a, 71 b}$, T. Farooque ${ }^{107}$, S. Farrell ${ }^{18}$, S.M. Farrington ${ }^{50}$, P. Farthouat ${ }^{36}$, F. Fassi ${ }^{35 e}$, P. Fassnacht ${ }^{36}$, D. Fassouliotis ${ }^{9}$, M. Faucci Giannelli ${ }^{50}$, W.J. Fawcett ${ }^{32}$, L. Fayard ${ }^{65}$, O.L. Fedin ${ }^{138, p}$, W. Fedorko ${ }^{175}$, M. Feickert ${ }^{42}$, L. Feligioni ${ }^{102}$, A. Fell ${ }^{149}$, C. Feng ${ }^{60 b}$, E.J. Feng ${ }^{36}$, M. Feng ${ }^{49}$, M.J. Fenton ${ }^{57}$, A.B. Fenyuk ${ }^{123}$, J. Ferrando ${ }^{46}$, A. Ferrante ${ }^{173}$, A. Ferrari ${ }^{172}$, P. Ferrari ${ }^{120}$, R. Ferrari ${ }^{71 a}$, D.E. Ferreira de Lima $^{61 b}$, A. Ferrer ${ }^{174}$, D. Ferrere ${ }^{54}$, C. Ferretti ${ }^{106}$, 
F. Fiedler ${ }^{100}$, A. Filipčič ${ }^{92}$, F. Filthaut ${ }^{119}$, K.D. Finelli ${ }^{25}$, M.C.N. Fiolhais ${ }^{140 a, 140 c, a}$, L. Fiorini ${ }^{174}$, F. Fischer ${ }^{114}$, W.C. Fisher ${ }^{107}$, I. Fleck ${ }^{151}$, P. Fleischmann ${ }^{106}$, R.R.M. Fletcher ${ }^{137}$, T. Flick ${ }^{182}$, B.M. Flierl ${ }^{114}$, L. Flores ${ }^{137}$, L.R. Flores Castillo ${ }^{63 a}$, F.M. Follega ${ }^{76 a, 76 b}$, N. Fomin ${ }^{17}$, J.H. Foo ${ }^{167}$, G.T. Forcolin ${ }^{76 a, 76 b}$, A. Formica ${ }^{145}$, F.A. Förster ${ }^{14}$, A.C. Forti ${ }^{101}$, A.G. Foster $^{21}$, M.G. Foti ${ }^{135}$, D. Fournier ${ }^{65}$, H. Fox ${ }^{90}$, P. Francavilla ${ }^{72 a, 72 b}$, S. Francescato ${ }^{73 a, 73 b}$, M. Franchini ${ }^{23 b, 23 a}$, S. Franchino ${ }^{61 a}$, D. Francis ${ }^{36}$, L. Franconi ${ }^{20}$, M. Franklin ${ }^{59}$, A.N. Fray ${ }^{93}$, P.M. Freeman ${ }^{21}$, B. Freund ${ }^{110}$, W.S. Freund ${ }^{81 b}$, E.M. Freundlich ${ }^{47}$, D.C. Frizzell ${ }^{129}$, D. Froidevaux ${ }^{36}$, J.A. Frost ${ }^{135}$, C. Fukunaga ${ }^{164}$, E. Fullana Torregrosa ${ }^{174}$, E. Fumagalli ${ }^{55 b, 55 a}$, T. Fusayasu ${ }^{116}$, J. Fuster ${ }^{174}$, A. Gabrielli ${ }^{23 b}$,23a , A. Gabrielli ${ }^{18}$, G.P. Gach ${ }^{84 a}$, S. Gadatsch $^{54}$, P. Gadow ${ }^{115}$, G. Gagliardi ${ }^{55 b, 55 a}$, L.G. Gagnon ${ }^{110}$, C. Galea ${ }^{27 b}$, B. Galhardo ${ }^{140 a}$, G.E. Gallardo ${ }^{135}$, E.J. Gallas ${ }^{135}$, B.J. Gallop ${ }^{144}$, G. Galster ${ }^{40}$, R. Gamboa Goni ${ }^{93}$, K.K. Gan ${ }^{127}$, S. Ganguly ${ }^{180}$, J. Gao ${ }^{60 a}$, Y. Gao ${ }^{50}$, Y.S. Gao ${ }^{31, m}$, C. García ${ }^{174}$, J.E. García Navarro ${ }^{174}$, J.A. García Pascual ${ }^{15 a}$, C. Garcia-Argos ${ }^{52}$,

M. Garcia-Sciveres ${ }^{18}$, R.W. Gardner ${ }^{37}$, N. Garelli ${ }^{153}$, S. Gargiulo ${ }^{52}$, V. Garonne ${ }^{134}$, A. Gaudiello ${ }^{55 b, 55 a}$, G. Gaudio ${ }^{71 a}$, I.L. Gavrilenko ${ }^{111}$, A. Gavrilyuk ${ }^{124}$, C. Gay ${ }^{175}$, G. Gaycken ${ }^{46}$, E.N. Gazis ${ }^{10}$, A.A. Geanta ${ }^{27 b}$, C.M. Gee ${ }^{146}$, C.N.P. Gee ${ }^{144}$, J. Geisen ${ }^{53}$, M. Geisen ${ }^{100}$, M.P. Geisler ${ }^{61 a}$, C. Gemme ${ }^{55 b}$, M.H. Genest ${ }^{58}$, C. Geng ${ }^{106}$, S. Gentile ${ }^{73 a, 73 b}$, S. George ${ }^{94}$, T. Geralis ${ }^{44}$, L.O. Gerlach ${ }^{53}$, P. Gessinger-Befurt ${ }^{100}$, G. Gessner ${ }^{47}$, S. Ghasemi ${ }^{151}$,

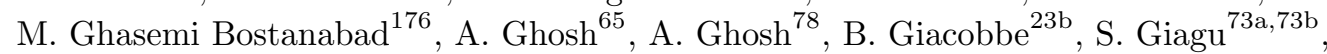
N. Giangiacomi ${ }^{23 b, 23 a}$, P. Giannetti ${ }^{72 a}$, A. Giannini ${ }^{70 a, 70 b}$, G. Giannini ${ }^{14}$, S.M. Gibson ${ }^{94}$, M. Gignac ${ }^{146}$, D. Gillberg ${ }^{34}$, G. Gilles ${ }^{182}$, D.M. Gingrich ${ }^{3, a x}$, M.P. Giordani ${ }^{67 a, 67 c}$, F.M. Giorgi ${ }^{23 b}$, P.F. Giraud ${ }^{145}$, G. Giugliarelli ${ }^{67 a, 67 c}$, D. Giugni ${ }^{69 a}$, F. Giuli ${ }^{74 a, 74 b}$, S. Gkaitatzis ${ }^{162}$, I. Gkialas ${ }^{9, h}$, E.L. Gkougkousis ${ }^{14}$, P. Gkountoumis ${ }^{10}$, L.K. Gladilin ${ }^{113}$, C. Glasman ${ }^{99}$, J. Glatzer ${ }^{14}$, P.C.F. Glaysher ${ }^{46}$, A. Glazov ${ }^{46}$, G.R. Gledhill ${ }^{132}$, M. Goblirsch-Kolb ${ }^{26}$, D. Godin ${ }^{110}$, S. Goldfarb ${ }^{105}$, T. Golling ${ }^{54}$, D. Golubkov ${ }^{123}$, A. Gomes ${ }^{140 a, 140 b}$, R. Goncalves Gama ${ }^{53}$, R. Gonçalo ${ }^{140 a}$, G. Gonella ${ }^{52}$, L. Gonella ${ }^{21}$, A. Gongadze ${ }^{80}$, F. Gonnella ${ }^{21}$, J.L. Gonski ${ }^{59}$, S. González de la $\mathrm{Hoz}^{174}$, S. Gonzalez-Sevilla ${ }^{54}$, G.R. Gonzalvo Rodriguez ${ }^{174}$, L. Goossens ${ }^{36}$, P.A. Gorbounov ${ }^{124}$, H.A. Gordon ${ }^{29}$, B. Gorini ${ }^{36}$, E. Gorini ${ }^{68 a, 68 b}$, A. Gorišek ${ }^{92}$, A.T. Goshaw ${ }^{49}$, M.I. Gostkin ${ }^{80}$, C.A. Gottardo ${ }^{119}$, M. Gouighri ${ }^{35 b}$, D. Goujdami ${ }^{35 c}$, A.G. Goussiou ${ }^{148}$, N. Govender ${ }^{33 c}$, C. Goy $^{5}$, E. Gozani ${ }^{160}$, I. Grabowska-Bold ${ }^{84 a}$, E.C. Graham ${ }^{91}$, J. Gramling ${ }^{171}$, E. Gramstad ${ }^{134}$, S. Grancagnolo ${ }^{19}$, M. Grandi ${ }^{156}$, V. Gratchev ${ }^{138}$, P.M. Gravila ${ }^{27 f}$, F.G. Gravili ${ }^{68 a, 68 b}$, C. Gray $^{57}$, H.M. Gray ${ }^{18}$, C. Grefe ${ }^{24}$, K. Gregersen ${ }^{97}$, I.M. Gregor ${ }^{46}$, P. Grenier ${ }^{153}$, K. Grevtsov ${ }^{46}$, C. Grieco ${ }^{14}$, N.A. Grieser ${ }^{129}$, J. Griffiths ${ }^{8}$, A.A. Grillo ${ }^{146}$, K. Grimm ${ }^{31,1}$, S. Grinstein ${ }^{14, \text { aa }}$, J.-F. Grivaz ${ }^{65}$, S. Groh ${ }^{100}$, E. Gross ${ }^{180}$, J. Grosse-Knetter ${ }^{53}$, Z.J. Grout ${ }^{95}$, C. Grud ${ }^{106}$, A. Grummer ${ }^{118}$, L. Guan ${ }^{106}$, W. Guan ${ }^{181}$, J. Guenther ${ }^{36}$, A. Guerguichon ${ }^{65}$, J.G.R. Guerrero Rojas ${ }^{174}$, F. Guescini ${ }^{115}$, D. Guest ${ }^{171}$, R. Gugel ${ }^{52}$, T. Guillemin ${ }^{5}$, S. Guindon ${ }^{36}$, U. Gul ${ }^{57}$, J. Guo $^{60 \mathrm{c}}, \mathrm{W} . \mathrm{Guo}^{106}$, Y. Guo ${ }^{60 \mathrm{a}, \mathrm{t}}$, Z. Guo ${ }^{102}$,

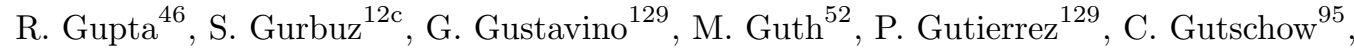
C. Guyot $^{145}$, C. Gwenlan ${ }^{135}$, C.B. Gwilliam ${ }^{91}$, A. Haas $^{125}$, C. Haber ${ }^{18}$, H.K. Hadavand ${ }^{8}$,

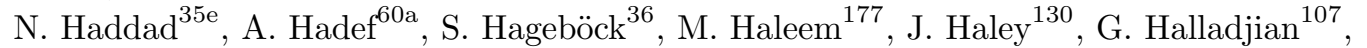
G.D. Hallewell ${ }^{102}$, K. Hamacher ${ }^{182}$, P. Hamal ${ }^{131}$, K. Hamano ${ }^{176}$, H. Hamdaoui ${ }^{35 e}$,

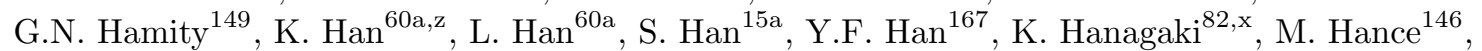
D.M. Handl ${ }^{114}$, B. Haney ${ }^{137}$, R. Hankache ${ }^{136}$, E. Hansen ${ }^{97}$, J.B. Hansen ${ }^{40}$, J.D. Hansen ${ }^{40}$, M.C. Hansen ${ }^{24}$, P.H. Hansen ${ }^{40}$, E.C. Hanson ${ }^{101}$, K. Hara ${ }^{169}$, T. Harenberg ${ }^{182}$, S. Harkusha ${ }^{108}$,

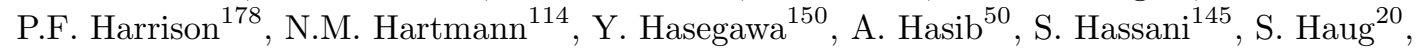
R. Hauser ${ }^{107}$, L.B. Havener ${ }^{39}$, M. Havranek ${ }^{142}$, C.M. Hawkes ${ }^{21}$, R.J. Hawkings ${ }^{36}$, D. Hayden ${ }^{107}$, C. Hayes ${ }^{155}$, R.L. Hayes ${ }^{175}$, C.P. Hays ${ }^{135}$, J.M. Hays ${ }^{93}$, H.S. Hayward ${ }^{91}$, S.J. Haywood ${ }^{144}$,

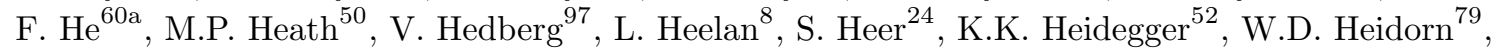

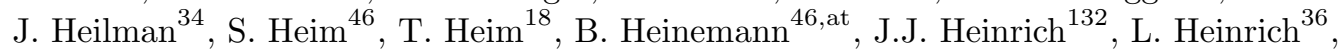




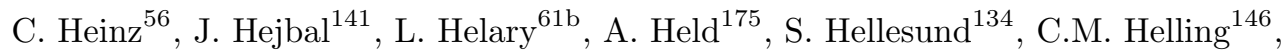
S. Hellman ${ }^{45 a, 45 b}$, C. Helsens ${ }^{36}$, R.C.W. Henderson ${ }^{90}$, Y. Heng ${ }^{181}$, S. Henkelmann ${ }^{175}$, A.M. Henriques Correia ${ }^{36}$, G.H. Herbert ${ }^{19}$, H. $\operatorname{Herde}^{26}$, V. Herget ${ }^{177}$, Y. Hernández Jiménez ${ }^{33 e}$, H. Herr ${ }^{100}$, M.G. Herrmann ${ }^{114}$, T. Herrmann ${ }^{48}$, G. Herten ${ }^{52}$, R. Hertenberger ${ }^{114}$, L. Hervas ${ }^{36}$, T.C. Herwig ${ }^{137}$, G.G. Hesketh ${ }^{95}$, N.P. Hessey ${ }^{168 a}$, A. Higashida ${ }^{163}$, S. Higashino ${ }^{82}$, E. Higón-Rodriguez ${ }^{174}$, K. Hildebrand ${ }^{37}$, E. Hill ${ }^{176}$, J.C. Hill ${ }^{32}$, K.K. Hill ${ }^{29}$, K.H. Hiller ${ }^{46}$,

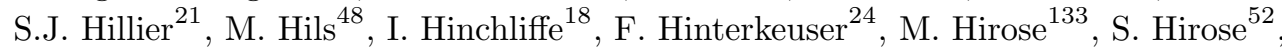

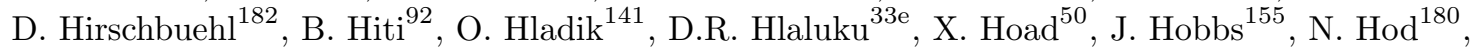
M.C. Hodgkinson ${ }^{149}$, A. Hoecker ${ }^{36}$, F. Hoenig ${ }^{14}$, D. Hohn ${ }^{52}$, D. Hohov ${ }^{65}$, T.R. Holmes ${ }^{37}$, M. Holzbock ${ }^{114}$, L.B.A.H. Hommels ${ }^{32}$, S. Honda ${ }^{169}$, T.M. Hong ${ }^{139}$, A. Hönle ${ }^{115}$,

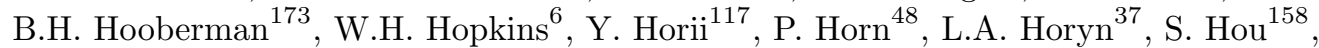

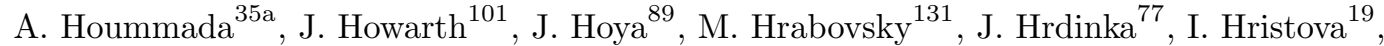
J. Hrivnac ${ }^{65}$, A. Hrynevich ${ }^{109}$, T. Hryn'ova ${ }^{5}$, P.J. $\mathrm{Hsu}^{64}$, S.-C. $\mathrm{Hsu}^{148}$, Q. $\mathrm{Hu}^{29}$, S. Hu ${ }^{60 \mathrm{c}}$,

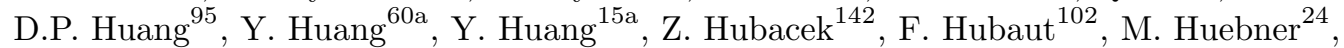
F. Huegging ${ }^{24}$, T.B. Huffman ${ }^{135}$, M. Huhtinen ${ }^{36}$, R.F.H. Hunter ${ }^{34}$, P. Huo ${ }^{155}$, A.M. Hupe ${ }^{34}$,

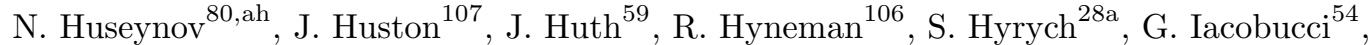

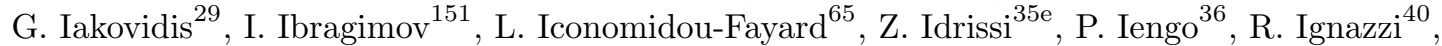

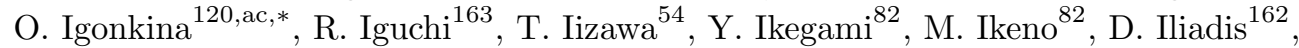

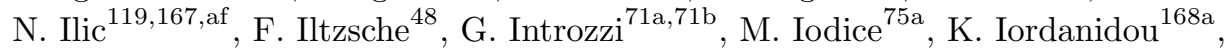

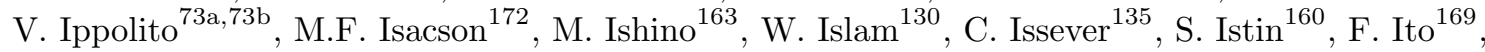

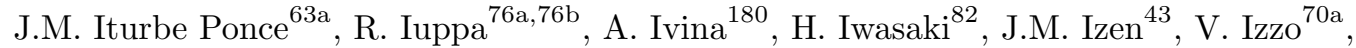
P. Jacka ${ }^{141}$, P. Jackson ${ }^{1}$, R.M. Jacobs ${ }^{24}$, B.P. Jaeger ${ }^{152}$, V. Jain ${ }^{2}$, G. Jäkel ${ }^{182}$, K.B. Jakobi ${ }^{100}$, K. Jakobs ${ }^{52}$, S. Jakobsen ${ }^{77}$, T. Jakoubek ${ }^{141}$, J. Jamieson ${ }^{57}$, K.W. Janas ${ }^{84 a}$, R. Jansky ${ }^{54}$, J. Janssen ${ }^{24}$, M. Janus ${ }^{53}$, P.A. Janus ${ }^{84 a}$, G. Jarlskog ${ }^{97}$, N. Javadov ${ }^{80, a h}$, T. Javurrek ${ }^{36}$, M. Javurkova ${ }^{52}$, F. Jeanneau ${ }^{145}$, L. Jeanty ${ }^{132}$, J. Jejelava ${ }^{159 a, a i}$, A. Jelinskas ${ }^{178}$, P. Jenni ${ }^{52, b}$, J. Jeong ${ }^{46}$, N. Jeong ${ }^{46}$, S. Jézéquel ${ }^{5}$, H. Ji ${ }^{181}$, J. Jia ${ }^{155}$, H. Jiang ${ }^{79}$, Y. Jiang ${ }^{60 a}$, Z. Jiang ${ }^{153, q}$, S. Jiggins ${ }^{52}$, F.A. Jimenez Morales ${ }^{38}$, J. Jimenez Pena ${ }^{115}$, S. Jin ${ }^{15 c}$, A. Jinaru ${ }^{27 b}$, O. Jinnouchi ${ }^{165}$, H. Jivan ${ }^{33 e}$, P. Johansson ${ }^{149}$, K.A. Johns ${ }^{7}$, C.A. Johnson ${ }^{66}$, K. Jon-And ${ }^{45 a, 45 b}$, R.W.L. Jones ${ }^{90}$, S.D. Jones ${ }^{156}$, S. Jones ${ }^{7}$, T.J. Jones ${ }^{91}$, J. Jongmanns ${ }^{61 a}$, P.M. Jorge ${ }^{140 a}$, J. Jovicevic ${ }^{36}$, X. Ju ${ }^{18}$, J.J. Junggeburth ${ }^{115}$, A. Juste Rozas ${ }^{14, \text { aa }}$, A. Kaczmarska ${ }^{85}$, M. Kado ${ }^{73 a, 73 b}$, H. Kagan ${ }^{127}$, M. Kagan ${ }^{153}$, C. Kahra ${ }^{100}$, T. Kaji ${ }^{179}$, E. Kajomovitz ${ }^{160}$, C.W. Kalderon ${ }^{97}$, A. Kaluza ${ }^{100}$ A. Kamenshchikov ${ }^{123}$, M. Kaneda ${ }^{163}$, L. Kanjir ${ }^{92}$, Y. Kano ${ }^{163}$, V.A. Kantserov ${ }^{112}$, J. Kanzaki ${ }^{82}$, L.S. Kaplan ${ }^{181}$, D. $\operatorname{Kar}^{33 e}$, K. Karava ${ }^{135}$, M.J. Kareem ${ }^{168 b}$, S.N. Karpov ${ }^{80}$, Z.M. Karpova ${ }^{80}$, V. Kartvelishvili ${ }^{90}$, A.N. Karyukhin ${ }^{123}$, L. Kashif ${ }^{181}$, R.D. Kass ${ }^{127}$, A. Kastanas ${ }^{45 a, 45 b}$ C. Kato ${ }^{60 d, 60 c}$, J. Katzy ${ }^{46}$, K. Kawade ${ }^{150}$, K. Kawagoe ${ }^{88}$, T. Kawaguchi ${ }^{117}$, T. Kawamoto ${ }^{163}$, G. Kawamura ${ }^{53}$, E.F. Kay ${ }^{176}$, V.F. Kazanin ${ }^{122 \mathrm{~b}, 122 \mathrm{a}}$, R. Keeler ${ }^{176}$, R. Kehoe ${ }^{42}$, J.S. Keller ${ }^{34}$, E. Kellermann ${ }^{97}$, D. Kelsey ${ }^{156}$, J.J. Kempster ${ }^{21}$, J. Kendrick ${ }^{21}$, O. Kepka ${ }^{141}$, S. Kersten ${ }^{182}$, B.P. Kerševan ${ }^{92}$, S. Ketabchi Haghighat ${ }^{167}$, M. Khader ${ }^{173}$, F. Khalil-Zada ${ }^{13}$, M. Khandoga ${ }^{145}$, A. Khanov ${ }^{130}$, A.G. Kharlamov ${ }^{122 \mathrm{~b}, 122 \mathrm{a}}$, T. Kharlamova ${ }^{122 \mathrm{~b}, 122 \mathrm{a}}$, E.E. Khoda ${ }^{175}$, A. Khodinov ${ }^{166}$, T.J. Khoo ${ }^{54}$, E. Khramov ${ }^{80}$, J. Khubua ${ }^{159 \mathrm{~b}}$, S. Kido ${ }^{83}$, M. Kiehn ${ }^{54}$, C.R. Kilby ${ }^{94}$, Y.K. Kim ${ }^{37}$, N. Kimura ${ }^{95}$, O.M. Kind ${ }^{19}$, B.T. King ${ }^{91, *}$, D. Kirchmeier ${ }^{48}$, J. Kirk ${ }^{144}$, A.E. Kiryunin ${ }^{115}$, T. Kishimoto ${ }^{163}$, D.P. Kisliuk ${ }^{167}$, V. Kitali ${ }^{46}$, O. Kivernyk ${ }^{5}$, T. Klapdor-Kleingrothaus ${ }^{52}$, M. Klassen ${ }^{61 a}$, M.H. Klein ${ }^{106}$, M. Klein ${ }^{91}$, U. Klein ${ }^{91}$, K. Kleinknecht ${ }^{100}$, P. Klimek ${ }^{121}$, A. Klimentov ${ }^{29}$, T. Klingl ${ }^{24}$, T. Klioutchnikova ${ }^{36}$, F.F. Klitzner ${ }^{114}$, P. Kluit ${ }^{120}$, S. Kluth ${ }^{115}$, E. Kneringer ${ }^{77}$, E.B.F.G. Knoops ${ }^{102}$, A. Knue ${ }^{52}$, D. Kobayashi ${ }^{88}$, T. Kobayashi ${ }^{163}$, M. Kobel ${ }^{48}$, M. Kocian ${ }^{153}$, P. Kodys ${ }^{143}$, P.T. Koenig ${ }^{24}$, T. Koffas ${ }^{34}$, N.M. Köhler ${ }^{36}$, T. Koi ${ }^{153}$, M. Kolb ${ }^{61 b}$, I. Koletsou ${ }^{5}$, T. Komarek ${ }^{131}$, T. Kondo ${ }^{82}$, N. Kondrashova ${ }^{60 c}$, K. Köneke ${ }^{52}$, A.C. König ${ }^{119}$, T. Kono ${ }^{126}$, R. Konoplich ${ }^{125, \text { ao }}$, V. Konstantinides ${ }^{95}$, N. Konstantinidis ${ }^{95}$, B. Konya ${ }^{97}$, 
R. Kopeliansky ${ }^{66}$, S. Koperny ${ }^{84 a}$, K. Korcyl ${ }^{85}$, K. Kordas ${ }^{162}$, G. Koren ${ }^{161}$, A. Korn ${ }^{95}$, I. Korolkov $^{14}$, E.V. Korolkova ${ }^{149}$, N. Korotkova ${ }^{113}$, O. Kortner ${ }^{115}$, S. Kortner ${ }^{115}$, T. Kosek ${ }^{143}$, V.V. Kostyukhin ${ }^{166}$, A. Kotwal ${ }^{49}$, A. Koulouris ${ }^{10}$, A. Kourkoumeli-Charalampidi ${ }^{71 a, 71 b}$, C. Kourkoumelis ${ }^{9}$, E. Kourlitis ${ }^{149}$, V. Kouskoura ${ }^{29}$, A.B. Kowalewska ${ }^{85}$, R. Kowalewski ${ }^{176}$, C. Kozakai ${ }^{163}$, W. Kozanecki ${ }^{145}$, A.S. Kozhin ${ }^{123}$, V.A. Kramarenko ${ }^{113}$, G. Kramberger ${ }^{92}$, D. Krasnopevtsev ${ }^{60 a}$, M.W. Krasny ${ }^{136}$, A. Krasznahorkay ${ }^{36}$, D. Krauss ${ }^{115}$, J.A. Kremer ${ }^{84 a}$, J. Kretzschmar ${ }^{91}$, P. Krieger ${ }^{167}$, F. Krieter ${ }^{114}$, A. Krishnan ${ }^{61 b}$, K. Krizka ${ }^{18}$, K. Kroeninger ${ }^{47}$,

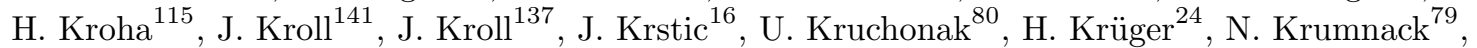
M.C. Kruse ${ }^{49}$, J.A. Krzysiak ${ }^{85}$, T. Kubota ${ }^{105}$, O. Kuchinskaia ${ }^{166}$, S. Kuday ${ }^{4 b}$, J.T. Kuechler ${ }^{46}$,

S. Kuehn ${ }^{36}$, A. Kugel ${ }^{61 \mathrm{a}}$, T. Kuhl ${ }^{46}$, V. Kukhtin ${ }^{80}$, R. Kukla ${ }^{102}$, Y. Kulchitsky ${ }^{108, a l}$,

S. Kuleshov ${ }^{147 d}$, Y.P. Kulinich ${ }^{173}$, M. Kuna ${ }^{58}$, T. Kunigo ${ }^{86}$, A. Kupco ${ }^{141}$, T. Kupfer ${ }^{47}$, O. Kuprash $^{52}$, H. Kurashige ${ }^{83}$, L.L. Kurchaninov ${ }^{168 a}$, Y.A. Kurochkin ${ }^{108}$, A. Kurova ${ }^{112}$, M.G. Kurth ${ }^{15 a, 15 d}$, E.S. Kuwertz ${ }^{36}$, M. Kuze ${ }^{165}$, A.K. Kvam ${ }^{148}$, J. Kvita ${ }^{131}$, T. Kwan ${ }^{104}$,

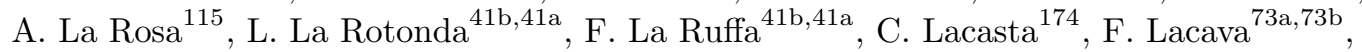
D.P.J. Lack $^{101}$, H. Lacker ${ }^{19}$, D. Lacour ${ }^{136}$, E. Ladygin ${ }^{80}$, R. Lafaye ${ }^{5}$, B. Laforge ${ }^{136}$, T. Lagouri ${ }^{33 e}$, S. Lai ${ }^{53}$, S. Lammers ${ }^{66}$, W. Lampl ${ }^{7}$, C. Lampoudis ${ }^{162}$, E. Lançon ${ }^{29}$, U. Landgraf ${ }^{52}$, M.P.J. Landon ${ }^{93}$, M.C. Lanfermann ${ }^{54}$, V.S. Lang $^{46}$, J.C. Lange ${ }^{53}$, R.J. Langenberg ${ }^{36}$,

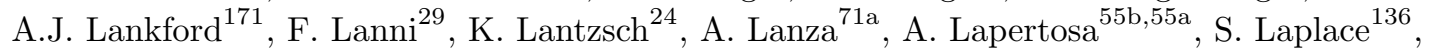
J.F. Laporte ${ }^{145}$, T. Lari ${ }^{69 a}$, F. Lasagni Manghi ${ }^{23 b, 23 a}$, M. Lassnig ${ }^{36}$, T.S. Lau ${ }^{63 a}$, A. Laudrain ${ }^{65}$, A. Laurier $^{34}$, M. Lavorgna ${ }^{70 a, 70 b}$, S.D. Lawlor ${ }^{94}$, M. Lazzaroni ${ }^{69 a, 69 b}$, B. Le ${ }^{105}$, E. Le Guirriec ${ }^{102}$, M. LeBlanc ${ }^{7}$, T. LeCompte ${ }^{6}$, F. Ledroit-Guillon ${ }^{58}$, A.C.A. Lee ${ }^{95}$, C.A. Lee ${ }^{29}$, G.R. Lee ${ }^{17}$, L. Lee ${ }^{59}$, S.C. Lee ${ }^{158}$, S.J. Lee ${ }^{34}$, B. Lefebvre ${ }^{168 a}$, M. Lefebvre ${ }^{176}$, F. Legger ${ }^{114}$, C. Leggett ${ }^{18}$, K. Lehmann ${ }^{152}$, N. Lehmann ${ }^{182}$, G. Lehmann Miotto ${ }^{36}$, W.A. Leight ${ }^{46}$, A. Leisos ${ }^{162, y}$, M.A.L. Leite ${ }^{81 d}$, C.E. Leitgeb ${ }^{114}$, R. Leitner ${ }^{143}$, D. Lellouch ${ }^{180, *}$, K.J.C. Leney ${ }^{42}$, T. Lenz ${ }^{24}$, B. Lenzi $^{36}$, R. Leone ${ }^{7}$, S. Leone ${ }^{72 a}$, C. Leonidopoulos ${ }^{50}$, A. Leopold ${ }^{136}$, G. Lerner ${ }^{156}$, C. Leroy ${ }^{110}$, R. Les $^{167}$, C.G. Lester ${ }^{32}$, M. Levchenko ${ }^{138}$, J. Levêque ${ }^{5}$, D. Levin ${ }^{106}$, L.J. Levinson ${ }^{180}$,

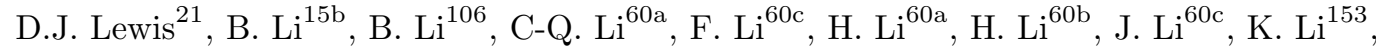

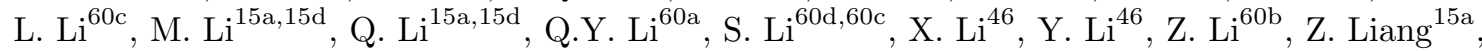

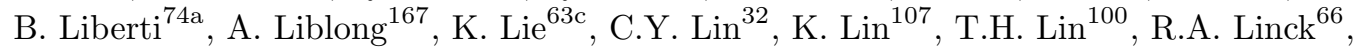
J.H. Lindon ${ }^{21}$, A.L. Lionti ${ }^{54}$, E. Lipeles ${ }^{137}$, A. Lipniacka ${ }^{17}$, M. Lisovyi ${ }^{61 b}$, T.M. Liss ${ }^{173, \text { av }}$, A. Lister $^{175}$, A.M. Litke ${ }^{146}$, J.D. Little ${ }^{8}$, B. Liu ${ }^{79}$, B.L. Liu ${ }^{6}$, H.B. Liu ${ }^{29}$, H. Liu ${ }^{106}$, J.B. Liu ${ }^{60 a}$, J.K.K. Liu ${ }^{135}$, K. Liu ${ }^{136}$, M. Liu ${ }^{60 a}$, P. Liu ${ }^{18}$, Y. Liu ${ }^{15 a, 15 d}$, Y.L. Liu ${ }^{106}$, Y.W. Liu ${ }^{60 a}$, M. Livan $^{71 \mathrm{a}, 71 \mathrm{~b}}$, A. Lleres $^{58}$, J. Llorente Merino ${ }^{152}$, S.L. Lloyd ${ }^{93}$, C.Y. Lo ${ }^{63 \mathrm{~b}}$, F. Lo Sterzo ${ }^{42}$, E.M. Lobodzinska ${ }^{46}$, P. Loch $^{7}$, S. Loffredo ${ }^{74 a, 74 b}$, T. Lohse ${ }^{19}$, K. Lohwasser ${ }^{149}$, M. Lokajicek ${ }^{141}$, J.D. Long ${ }^{173}$, R.E. Long ${ }^{90}$, L. Longo ${ }^{36}$, K.A. Looper ${ }^{127}$, J.A. Lopez ${ }^{14 d}$, I. Lopez Paz ${ }^{101}$, A. Lopez Solis ${ }^{149}$, J. Lorenz ${ }^{114}$, N. Lorenzo Martinez ${ }^{5}$, M. Losada $^{22 a}$, P.J. Lösel ${ }^{114}$, A. Lösle ${ }^{52}$, X. Lou ${ }^{46}$, X. Lou ${ }^{15 a}$, A. Lounis ${ }^{65}$, J. Love ${ }^{6}$, P.A. Love ${ }^{90}$, J.J. Lozano Bahilo ${ }^{174}$, M. Lu ${ }^{60 a}$, Y.J. Lu ${ }^{64}$, H.J. Lubatti ${ }^{148}$, C. Luci ${ }^{73 a, 73 b}$, A. Lucotte ${ }^{58}$, C. Luedtke ${ }^{52}$, F. Luehring ${ }^{66}$, I. Luise ${ }^{136}$, L. Luminari ${ }^{73 a}$, B. Lund-Jensen ${ }^{154}$, M.S. Lutz ${ }^{103}$, D. Lynn ${ }^{29}$, R. Lysak ${ }^{141}$, E. Lytken ${ }^{97}$, F. Lyu ${ }^{15 a}$, V. Lyubushkin ${ }^{80}$, T. Lyubushkina ${ }^{80}$, H. $\mathrm{Ma}^{29}$, L.L. Ma ${ }^{60 \mathrm{~b}}$, Y. Ma ${ }^{60 \mathrm{~b}}$, G. Maccarrone ${ }^{51}$, A. Macchiolo ${ }^{115}$, C.M. Macdonald ${ }^{149}$, J. Machado Miguens ${ }^{137}$, D. Madaffari ${ }^{174}$, R. Madar ${ }^{38}$, W.F. Mader ${ }^{48}$, N. Madysa ${ }^{48}$, J. Maeda ${ }^{83}$, S. Maeland ${ }^{17}$, T. Maeno ${ }^{29}$, M. Maerker ${ }^{48}$, A.S. Maevskiy ${ }^{113}$, V. Magerl ${ }^{52}$, N. Magini ${ }^{79}$, D.J. Mahon ${ }^{39}$, C. Maidantchik ${ }^{81 b}$, T. Maier ${ }^{114}$, A. Maio ${ }^{140 a, 140 b, 140 d}$, K. Maj ${ }^{84 a}$, O. Majersky ${ }^{28 a}$, S. Majewski ${ }^{132}$, Y. Makida ${ }^{82}$, N. Makovec ${ }^{65}$, B. Malaescu ${ }^{136}$, Pa. Malecki ${ }^{85}$, V.P. Maleev ${ }^{138}$, F. Malek ${ }^{58}$, U. Mallik ${ }^{78}$, D. Malon ${ }^{6}$, C. Malone ${ }^{32}$, S. Maltezos ${ }^{10}$, S. Malyukov ${ }^{80}$, J. Mamuzic ${ }^{174}$, G. Mancini ${ }^{51}$, I. Mandić ${ }^{92}$,

L. Manhaes de Andrade Filho ${ }^{81 a}$, I.M. Maniatis ${ }^{162}$, J. Manjarres Ramos ${ }^{48}$, K.H. Mankinen ${ }^{97}$, A. Mann $^{114}$, A. Manousos ${ }^{77}$, B. Mansoulie ${ }^{145}$, I. Manthos ${ }^{162}$, S. Manzoni ${ }^{120}$, A. Marantis ${ }^{162}$, 
G. Marceca ${ }^{30}$, L. Marchese ${ }^{135}$, G. Marchiori ${ }^{136}$, M. Marcisovsky ${ }^{141}$, C. Marcon ${ }^{97}$, C.A. Marin Tobon ${ }^{36}$, M. Marjanovic ${ }^{129}$, Z. Marshall ${ }^{18}$, M.U.F. Martensson ${ }^{172}$, S. Marti-Garcia ${ }^{174}$, C.B. Martin ${ }^{127}$, T.A. Martin ${ }^{178}$, V.J. Martin ${ }^{50}$, B. Martin dit Latour ${ }^{17}$, L. Martinelli ${ }^{75 a, 75 b}$, M. Martinez ${ }^{14, \text { aa }}$, V.I. Martinez Outschoorn ${ }^{103}$, S. Martin-Haugh ${ }^{144}$, V.S. Martoiu ${ }^{27 b}$, A.C. Martyniuk ${ }^{95}$, A. Marzin ${ }^{36}$, S.R. Maschek ${ }^{115}$, L. Masetti ${ }^{100}$, T. Mashimo ${ }^{163}$, R. Mashinistov ${ }^{111}$, J. Masik ${ }^{101}$, A.L. Maslennikov ${ }^{122 b, 122 a}$, L. Massa ${ }^{74 a, 74 b}$, P. Massarotti ${ }^{70 a, 70 b}$, P. Mastrandrea ${ }^{72 a, 72 b}$, A. Mastroberardino ${ }^{41 b, 41 a}$, T. Masubuchi ${ }^{163}$, D. Matakias ${ }^{10}$, A. Matic ${ }^{114}$, P. Mättig ${ }^{24}$, J. Maurer ${ }^{27 b}$, B. Maček ${ }^{92}$, D.A. Maximov ${ }^{122 b, 122 a}$, R. Mazini ${ }^{158}$, I. Maznas ${ }^{162}$, S.M. Mazza ${ }^{146}$, S.P. Mc Kee ${ }^{106}$, T.G. McCarthy ${ }^{115}$, W.P. McCormack ${ }^{18}$, E.F. McDonald ${ }^{105}$, J.A. Mcfayden ${ }^{36}$, G. Mchedlidze ${ }^{159 b}$, M.A. McKay ${ }^{42}$, K.D. McLean ${ }^{176}$, S.J. McMahon ${ }^{144}$, P.C. $\mathrm{McNamara}^{105}$, C.J. $\mathrm{McNicol}^{178}$, R.A. McPherson ${ }^{176, \text { af }}$, J.E. Mdhluli ${ }^{33 \mathrm{e}}$, Z.A. Meadows ${ }^{103}$, S. Meehan ${ }^{36}$, T. Megy ${ }^{52}$, S. Mehlhase ${ }^{114}$, A. Mehta $^{91}$, T. Meideck ${ }^{58}$, B. Meirose ${ }^{43}$, D. Melini ${ }^{174}$ B.R. Mellado Garcia ${ }^{33 e}$, J.D. Mellenthin ${ }^{53}$, M. Melo ${ }^{28 a}$, F. Meloni ${ }^{46}$, A. Melzer ${ }^{24}$, S.B. Menary ${ }^{101}$, E.D. Mendes Gouveia ${ }^{140 a, 140 e}$, L. Meng ${ }^{36}$, X.T. Meng ${ }^{106}$, S. Menke ${ }^{115}$, E. Meoni ${ }^{41 b, 41 a}$, S. Mergelmeyer ${ }^{19}$, S.A.M. Merkt ${ }^{139}$, C. Merlassino ${ }^{20},{\text { P. } \text { Mermod }^{54}, \text { L. Merola }}^{70 a, 70 b}$, C. Meroni ${ }^{69 a}$, O. Meshkov ${ }^{113,111}$, J.K.R. Meshreki ${ }^{151}$, A. Messina ${ }^{73 a, 73 b}$, J. Metcalfe ${ }^{6}$, A.S. Mete ${ }^{171}$, C. Meyer ${ }^{66}$, J. Meyer ${ }^{160}$, J-P. Meyer ${ }^{145}$, H. Meyer Zu Theenhausen ${ }^{61 a}$, F. Miano ${ }^{156}$, M. Michetti ${ }^{19}$, R.P. Middleton ${ }^{144}$, L. Mijović ${ }^{50}$, G. Mikenberg ${ }^{180}$, M. Mikestikova ${ }^{141}$, M. Mikuž ${ }^{92}$, H. Mildner ${ }^{149}$, M. Milesi ${ }^{105}$, A. Milic ${ }^{167}$, D.A. Millar ${ }^{93}$, D.W. Miller ${ }^{37}$, A. Milov ${ }^{180}$,

D.A. Milstead ${ }^{45 a, 45 b}$, R.A. Mina ${ }^{153, \mathrm{q}}$, A.A. Minaenko ${ }^{123}$, M. Miñano Moya ${ }^{174}$, I.A. Minashvili ${ }^{159 b}$, A.I. Mincer ${ }^{125}$, B. Mindur ${ }^{84 a}$, M. Mineev ${ }^{80}$, Y. Minegishi ${ }^{163}$, L.M. Mirr $^{14}$, A. Mirto ${ }^{68 a, 68 b}$, K.P. Mistry ${ }^{137}$, T. Mitani ${ }^{179}$, J. Mitrevski ${ }^{114}$, V.A. Mitsou ${ }^{174}$, M. Mittal ${ }^{60 c}$, O. Miu ${ }^{167}$, A. Miucci ${ }^{20}$, P.S. Miyagawa ${ }^{149}$, A. Mizukami ${ }^{82}$, J.U. Mjörnmark ${ }^{97}$, T. Mkrtchyan ${ }^{184}$, M. Mlynarikova ${ }^{143}$, T. Moa ${ }^{45 a, 45 b}$, K. Mochizuki ${ }^{110},{\text { P. } \operatorname{Mogg}^{52}, \text { S. Mohapatra }}^{39}$, R. Moles-Valls ${ }^{24}$, M.C. Mondragon ${ }^{107}$, K. Mönig ${ }^{46}$, J. Monk ${ }^{40}$, E. Monnier ${ }^{102}$, A. Montalbano ${ }^{152}$,

J. Montejo Berlingen ${ }^{36}$, M. Montella ${ }^{95}$, F. Monticelli ${ }^{89}$, S. Monzani $^{69 a}$, N. Morange ${ }^{65}$, D. Moreno ${ }^{22 a}$, M. Moreno Llácer ${ }^{36}$, C. Moreno Martinez ${ }^{14}$, P. Morettini $^{55 b}$, M. Morgenstern ${ }^{120}$, S. Morgenstern ${ }^{48}$, D. Mori ${ }^{152}$, M. Morii ${ }^{59}$, M. Morinaga ${ }^{179}$, V. Morisbak ${ }^{134}$, A.K. Morley ${ }^{36}$, G. Mornacchi ${ }^{36}$, A.P. Morris ${ }^{95}$, L. Morvaj ${ }^{155}$, P. Moschovakos ${ }^{36}$, B. Moser ${ }^{120}$, M. Mosidze ${ }^{159 b}$, T. Moskalets ${ }^{145}$, H.J. Moss ${ }^{149}$, J. Moss ${ }^{31, \mathrm{n}}$, E.J.W. Moyse ${ }^{103}$, S. Muanza ${ }^{102}$, J. Mueller ${ }^{139}$, R.S.P. Mueller ${ }^{114}$, D. Muenstermann ${ }^{90}$, G.A. Mullier ${ }^{97}$, J.L. Munoz Martinez ${ }^{14}$, F.J. Munoz Sanchez ${ }^{101}$, P. Murin ${ }^{28 b}$, W.J. Murray ${ }^{178,144}$, A. Murrone ${ }^{69 a, 69 b}$, M. Muškinja ${ }^{18}$, C. Mwewa ${ }^{33 a}$, A.G. Myagkov ${ }^{123, \text { ap }}$, J. Myers ${ }^{132}$, M. Myska ${ }^{142}$, B.P. Nachman ${ }^{18}$, O. Nackenhorst ${ }^{47}$, A.Nag Nag ${ }^{48}$, K. Nagai ${ }^{135}$, K. Nagano ${ }^{82}$, Y. Nagasaka ${ }^{62}$, M. Nagel ${ }^{52}$, J.L. Nagle ${ }^{29}$, E. Nagy ${ }^{102}$, A.M. Nairz ${ }^{36}$, Y. Nakahama ${ }^{117}$, K. Nakamura ${ }^{82}$, T. Nakamura ${ }^{163}$, I. Nakano ${ }^{128}$, H. Nanjo ${ }^{133}$, F. Napolitano ${ }^{61 \mathrm{a}}$, R.F. Naranjo Garcia ${ }^{46}$, R. Narayan ${ }^{42}$, I. Naryshkin ${ }^{138}$, T. Naumann ${ }^{46}$, G. Navarro ${ }^{22 a}$, H.A. Neal ${ }^{106, *}$, P.Y. Nechaeva ${ }^{111}$, F. Nechansky ${ }^{46}$, T.J. Neep ${ }^{21}$, A. Negri ${ }^{71 a, 71 b}$, M. Negrini ${ }^{23 b}$, C. Nellist ${ }^{53}$, M.E. Nelson ${ }^{135}$, S. Nemecek ${ }^{141}$, P. Nemethy ${ }^{125}$, M. Nessi ${ }^{36, d}$, M.S. Neubauer ${ }^{173}$, M. Neumann ${ }^{182}$, P.R. Newman ${ }^{21}$, Y.S. $\mathrm{Ng}^{19}$, Y.W.Y. $\mathrm{Ng}^{171}$, B. Ngair ${ }^{35 e}$, H.D.N. Nguyen ${ }^{102}$, T. Nguyen Manh $^{110}$, E. Nibigira ${ }^{38}$, R.B. Nickerson ${ }^{135}$, R. Nicolaidou ${ }^{145}$, D.S. Nielsen ${ }^{40}$, J. Nielsen ${ }^{146}$, N. Nikiforou ${ }^{11}$, V. Nikolaenko ${ }^{123, \text { ap }}$, I. Nikolic-Audit ${ }^{136}$, K. Nikolopoulos ${ }^{21}$, P. Nilsson ${ }^{29}$, H.R. Nindhito ${ }^{54}$, Y. Ninomiya ${ }^{82}$, A. Nisati ${ }^{73 a}$, N. Nishu ${ }^{60 c}$,

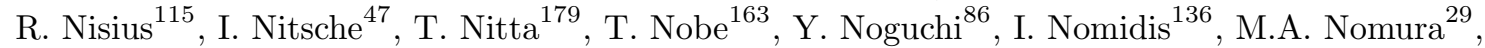

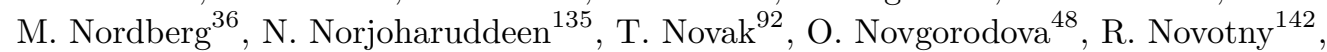

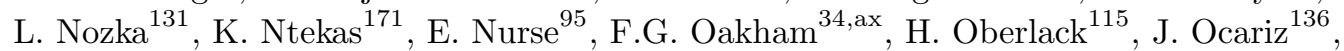
A. Ochi ${ }^{83}$, I. Ochoa ${ }^{39}$, J.P. Ochoa-Ricoux ${ }^{147 a}$, K. O'Connor ${ }^{26}$, S. Oda ${ }^{88}$, S. Odaka ${ }^{82}$, S. Oerdek ${ }^{53}$, A. Ogrodnik ${ }^{84 a}$, A. $\mathrm{Oh}^{101}$, S.H. $\mathrm{Oh}^{49}$, C.C. $\mathrm{Ohm}^{154}$, H. Oide ${ }^{165}$, M.L. Ojeda ${ }^{167}$, H. Okawa ${ }^{169}$, Y. Okazaki ${ }^{86}$, Y. Okumura ${ }^{163}$, T. Okuyama ${ }^{82}$, A. Olariu ${ }^{27 b}$, L.F. Oleiro Seabra ${ }^{140 a}$, 
S.A. Olivares Pino ${ }^{147 a}$, D. Oliveira Damazio ${ }^{29}$, J.L. Oliver ${ }^{1}$, M.J.R. Olsson ${ }^{171}$, A. Olszewski ${ }^{85}$, J. Olszowska ${ }^{85}$, D.C. O’Neil ${ }^{152}$, A.P. O'neill ${ }^{135}$, A. Onofre ${ }^{140 a, 140 e}$, P.U.E. Onyisi ${ }^{11}$, H. Oppen ${ }^{134}$, M.J. Oreglia ${ }^{37}$, G.E. Orellana ${ }^{89}$, D. Orestano ${ }^{75 a, 75 b}$, N. Orlando ${ }^{14}$, R.S. Orr ${ }^{167}$, V. O'Shea ${ }^{57}$, R. Ospanov ${ }^{60 a}$, G. Otero y Garzon ${ }^{30}$, H. Otono ${ }^{88}$, P.S. Ott ${ }^{61 \mathrm{a}}$, M. Ouchrif $^{35 \mathrm{~d}}$, J. Ouellette ${ }^{29}$, F. Ould-Saada ${ }^{134}$, A. Ouraou ${ }^{145}$, Q. Ouyang ${ }^{15 a}$, M. Owen ${ }^{57}$, R.E. Owen ${ }^{21}$, V.E. Ozcan ${ }^{12 c}$, N. Ozturk ${ }^{8}$, J. Pacalt ${ }^{131}$, H.A. Pacey ${ }^{32}$, K. Pachal ${ }^{49}$, A. Pacheco Pages ${ }^{14}$, C. Padilla Aranda ${ }^{14}$, S. Pagan Griso ${ }^{18}$, M. Paganini ${ }^{183}$, G. Palacino ${ }^{66}$, S. Palazzo ${ }^{50}$, S. Palestini ${ }^{36}$, M. Palka ${ }^{84 b}$, D. Pallin $^{38}$, I. Panagoulias ${ }^{10}$, C.E. Pandini ${ }^{36}$, J.G. Panduro Vazquez ${ }^{94}$, P. Pani ${ }^{46}$,

G. Panizzo ${ }^{67 a, 67 c}$, L. Paolozzi ${ }^{54}$, C. Papadatos ${ }^{110}$, K. Papageorgiou ${ }^{9, h}$, S. Parajuli ${ }^{43}$, A. Paramonov ${ }^{6}$, D. Paredes Hernandez ${ }^{63 b}$, S.R. Paredes Saenz ${ }^{135}$, B. Parida ${ }^{166}$, T.H. Park ${ }^{167}$ A.J. Parker ${ }^{31}$, M.A. Parker ${ }^{32}$, F. Parodi ${ }^{55 b, 55 a}$, E.W. Parrish ${ }^{121}$, J.A. Parsons ${ }^{39}$, U. Parzefall ${ }^{52}$, L. Pascual Dominguez ${ }^{136}$, V.R. Pascuzzi ${ }^{167}$, J.M.P. Pasner ${ }^{146}$, E. Pasqualucci ${ }^{73 a}$, S. Passaggio ${ }^{55 b}$, F. Pastore ${ }^{94}$, P. Pasuwan ${ }^{45 a, 45 b}$, S. Pataraia ${ }^{100}$, J.R. Pater ${ }^{101}$, A. Pathak ${ }^{181, j}$, T. Pauly ${ }^{36}$, B. Pearson ${ }^{115}$, M. Pedersen ${ }^{134}$, L. Pedraza Diaz ${ }^{119}$, R. Pedro ${ }^{140 a}$, T. Peiffer ${ }^{53}$, S.V. Peleganchuk ${ }^{122 b, 122 a}$, O. Penc ${ }^{141}$, H. Peng ${ }^{60 a}$, B.S. Peralva ${ }^{81 a}$, M.M. Perego ${ }^{65}$, A.P. Pereira Peixoto ${ }^{140 a}$, D.V. Perepelitsa ${ }^{29}$, F. Peri ${ }^{19}$, L. Perini ${ }^{69 a, 69 b}$, H. Pernegger ${ }^{36}$, S. Perrella ${ }^{70 a, 70 b}$, K. Peters ${ }^{46}$, R.F.Y. Peters ${ }^{101}$, B.A. Petersen ${ }^{36}$, T.C. Petersen ${ }^{40}$, E. Petit ${ }^{102}$, A. Petridis ${ }^{1}$, C. Petridou ${ }^{162}$, P. Petroff ${ }^{65}$, M. Petrov $^{135}$, F. Petrucci $^{75 a, 75 b}$, M. Pettee ${ }^{183}$, N.E. Pettersson ${ }^{103}$, K. Petukhova ${ }^{143}$, A. Peyaud ${ }^{145}$, R. Pezoa ${ }^{147 d}$, L. Pezzotti ${ }^{71 a, 71 b}$, T. Pham ${ }^{105}$, F.H. Phillips ${ }^{107}$, P.W. Phillips ${ }^{144}$, M.W. Phipps ${ }^{173}$, G. Piacquadio ${ }^{155}$, E. Pianori ${ }^{18}$, A. Picazio ${ }^{103}$, R.H. Pickles ${ }^{101}$, R. Piegaia ${ }^{30}$, D. Pietreanu ${ }^{27 b}$, J.E. Pilcher ${ }^{37}$, A.D. Pilkington ${ }^{101}$,

M. Pinamonti ${ }^{74 a, 74 b}$, J.L. Pinfold ${ }^{3}$, M. Pitt ${ }^{161}$, L. Pizzimento ${ }^{74 a, 74 b}$, M.-A. Pleier ${ }^{29}$, V. Pleskot ${ }^{143}$, E. Plotnikova ${ }^{80}$, P. Podberezko ${ }^{122 b, 122 a}$, R. Poettgen ${ }^{97}$, R. Poggi ${ }^{54}$, L. Poggioli ${ }^{65}$,

I. Pogrebnyak ${ }^{107}$, D. Pohl $^{24}$, I. Pokharel ${ }^{53}$, G. Polesello ${ }^{71 \mathrm{a}}$, A. Poley ${ }^{18}$, A. Policicchio ${ }^{73 a, 73 b}$, R. Polifka ${ }^{143}$, A. Polini ${ }^{23 b}$, C.S. Pollard ${ }^{46}$, V. Polychronakos ${ }^{29}$, D. Ponomarenko ${ }^{112}$, L. Pontecorvo ${ }^{36}$, S. Popa ${ }^{27 a}$, G.A. Popeneciu ${ }^{27 d}$, L. Portales ${ }^{5}$, D.M. Portillo Quintero ${ }^{58}$, S. Pospisil ${ }^{142}$, K. Potamianos ${ }^{46}$, I.N. Potrap ${ }^{80}$, C.J. Potter ${ }^{32}$, H. Potti ${ }^{11}$, T. Poulsen ${ }^{97}$, J. Poveda ${ }^{36}$, T.D. Powell ${ }^{149}$, G. Pownall ${ }^{46}$, M.E. Pozo Astigarraga ${ }^{36}$, P. Pralavorio ${ }^{102}$, S. Prell ${ }^{79}$, D. Price ${ }^{101}$, M. Primavera ${ }^{68 a}$, S. Prince ${ }^{104}$, M.L. Proffitt ${ }^{148}$, N. Proklova ${ }^{112}$, K. Prokofiev ${ }^{63 c}$, F. Prokoshin ${ }^{80}$, S. Protopopescu ${ }^{29}$, J. Proudfoot ${ }^{6}$, M. Przybycien ${ }^{84 a}$, D. Pudzha ${ }^{138}$, A. Puri ${ }^{173}$, P. Puzo ${ }^{65}$, J. Qian ${ }^{106}$, Y. Qin ${ }^{101}$, A. Quadt ${ }^{53}$, M. Queitsch-Maitland ${ }^{46}$, A. Qureshi ${ }^{1}$, M. Racko $^{28 a}$, P. Rados ${ }^{105}$, F. Ragusa ${ }^{69 a, 69 b}$, G. Rahal ${ }^{98}$, J.A. Raine ${ }^{54}$, S. Rajagopalan ${ }^{29}$, A. Ramirez Morales ${ }^{93}$, K. Ran ${ }^{15 a, 15 d}$, T. Rashid ${ }^{65}$, S. Raspopov ${ }^{5}$, D.M. Rauch ${ }^{46}$, F. Rauscher ${ }^{114}$, S. Rave ${ }^{100}$, B. Ravina ${ }^{149}$, I. Ravinovich ${ }^{180}$, J.H. Rawling ${ }^{101}$, M. Raymond ${ }^{36}$, A.L. Read $^{134}$, N.P. Readioff ${ }^{58}$, M. Reale ${ }^{68 a, 68 b}$, D.M. Rebuzzi ${ }^{71 a, 71 b}$, A. Redelbach ${ }^{177}$, G. Redlinger ${ }^{29}$, K. Reeves ${ }^{43}$, L. Rehnisch ${ }^{19}$, J. Reichert ${ }^{137}$, D. Reikher ${ }^{161}$, A. Reiss ${ }^{100}$, A. Rej ${ }^{151}$, C. Rembser ${ }^{36}$, M. Renda $^{27 b}$, M. Rescigno ${ }^{73 a}$, S. Resconi ${ }^{69 a}$, E.D. Resseguie ${ }^{137}$, S. Rettie ${ }^{175}$, E. Reynolds $^{21}$, O.L. Rezanova ${ }^{122 b, 122 a}$, P. Reznicek ${ }^{143}$, E. Ricci ${ }^{76 a, 76 b}$, R. Richter ${ }^{115}$, S. Richter ${ }^{46}$, E. Richter-Was ${ }^{84 b}$, O. Ricken ${ }^{24}$, M. Ridel ${ }^{136}$, P. Rieck ${ }^{115}$, C.J. Riegel ${ }^{182}$, O. Rifki ${ }^{46}$ M. Rijssenbeek ${ }^{155}$, A. Rimoldi ${ }^{71 a, 71 b}$, M. Rimoldi ${ }^{46}$, L. Rinaldi ${ }^{23 b}$, G. Ripellino ${ }^{154}$, I. Riu ${ }^{14}$, J.C. Rivera Vergara ${ }^{176}$, F. Rizatdinova ${ }^{130}$, E. Rizvi ${ }^{93}$, C. Rizzi ${ }^{36}$, R.T. Roberts ${ }^{101}$, S.H. Robertson ${ }^{104, \text { af }}$, M. Robin ${ }^{46}$, D. Robinson ${ }^{32}$, J.E.M. Robinson ${ }^{46}$, C.M. Robles Gajardo ${ }^{147 d}$, A. Robson ${ }^{57}$, A. Rocchi ${ }^{74 a, 74 b}$, E. Rocco ${ }^{100}$, C. Roda ${ }^{72 a, 72 b}$, S. Rodriguez Bosca ${ }^{174}$,

A. Rodriguez Perez ${ }^{14}$, D. Rodriguez Rodriguez ${ }^{174}$, A.M. Rodríguez Vera ${ }^{168 b}$, S. Roe ${ }^{36}$, O. Røhne ${ }^{134}$, R. Röhrig ${ }^{115}$, C.P.A. Roland ${ }^{66}$, J. Roloff ${ }^{59}$, A. Romaniouk ${ }^{112}$, M. Romano ${ }^{23 b, 23 a}$, N. Rompotis ${ }^{91}$, M. Ronzani ${ }^{125}$, L. Roos ${ }^{136}$, S. Rosati ${ }^{73 a}$, K. Rosbach ${ }^{52}$, G. Rosin ${ }^{103}$, B.J. Rosser ${ }^{137}$, E. Rossi ${ }^{46}$, E. Rossi ${ }^{75 a, 75 b}$, E. Rossi $^{70 a}, 70 \mathrm{~b}$, L.P. Rossi $^{55 b}$, L. Rossini $^{69 a}, 69 \mathrm{~b}$, R. Rosten ${ }^{14}$, M. Rotaru ${ }^{27 b}$, J. Rothberg ${ }^{148}$, D. Rousseau ${ }^{65}$, G. Rovelli ${ }^{71 \mathrm{a}, 71 \mathrm{~b}}$, A. Roy ${ }^{11}$, D. Roy $^{33 \mathrm{e}}$, 
A. Rozanov ${ }^{102}$, Y. Rozen ${ }^{160}$, X. Ruan ${ }^{33 e}$, F. Rubbo ${ }^{153}$, F. Rühr ${ }^{52}$, A. Ruiz-Martinez ${ }^{174}$, A. Rummler ${ }^{36}$, Z. Rurikova ${ }^{52}$, N.A. Rusakovich ${ }^{80}$, H.L. Russell ${ }^{104}$, L. Rustige ${ }^{38,47}$, J.P. Rutherfoord ${ }^{7}$, E.M. Rüttinger ${ }^{149}$, M. Rybar $^{39}$, G. Rybkin ${ }^{65}$, E.B. Rye ${ }^{134}$, A. Ryzhov ${ }^{123}$, P. Sabatini ${ }^{53}$, G. Sabato ${ }^{120}$, S. Sacerdoti ${ }^{65}$, H.F-W. Sadrozinski ${ }^{146}$, R. Sadykov ${ }^{80}$,

F. Safai Tehrani ${ }^{73 a}$, B. Safarzadeh Samani ${ }^{156}$, P. Saha ${ }^{121}$, S. Saha ${ }^{104}$, M. Sahinsoy ${ }^{61 a}$, A. Sahu ${ }^{182}$, M. Saimpert ${ }^{46}$, M. Saito ${ }^{163}$, T. Saito ${ }^{163}$, H. Sakamoto ${ }^{163}$, A. Sakharov ${ }^{125, \text { ao }}$, D. Salamani ${ }^{54}$, G. Salamanna ${ }^{75 a, 75 b}$, J.E. Salazar Loyola ${ }^{147 d}$, P.H. Sales De Bruin ${ }^{172}$, A. Salnikov ${ }^{153}$, J. Salt ${ }^{174}$, D. Salvatore ${ }^{41 \mathrm{~b}, 41 \mathrm{a}}$, F. Salvatore ${ }^{156}$, A. Salvucci ${ }^{63 a, 63 b, 63 c}$, A. Salzburger ${ }^{36}$, J. Samarati ${ }^{36}$,

D. Sammel ${ }^{52}$, D. Sampsonidis ${ }^{162}$, D. Sampsonidou ${ }^{162}$, J. Sánchez ${ }^{174}$, A. Sanchez Pineda ${ }^{67 a, 67 c}$, H. Sandaker ${ }^{134}$, C.O. Sander ${ }^{46}$, I.G. Sanderswood ${ }^{90}$, M. Sandhoff ${ }^{182}$, C. Sandoval ${ }^{22 a}$, D.P.C. Sankey ${ }^{144}$, M. Sannino ${ }^{55 b, 55 a}$, Y. Sano ${ }^{117}$, A. Sansoni ${ }^{51}$, C. Santoni ${ }^{38}$, H. Santos ${ }^{140 a, 140 b}$, S.N. Santpur ${ }^{18}$, A. Santra ${ }^{174}$, A. Sapronov ${ }^{80}$, J.G. Saraiva ${ }^{140 a, 140 d}$, O. Sasaki ${ }^{82}$, K. Sato ${ }^{169}$, F. Sauerburger ${ }^{52}$, E. Sauvan ${ }^{5}$, P. Savard ${ }^{167, a x}$, N. Savic ${ }^{115}$, R. Sawada ${ }^{163}$, C. Sawyer ${ }^{144}$, L. Sawyer ${ }^{96, \text { am }}$, C. Sbarra ${ }^{23 b}$, A. Sbrizzi ${ }^{23 a}$, T. Scanlon ${ }^{95}$, J. Schaarschmidt ${ }^{148}$, P. Schacht ${ }^{115}$, B.M. Schachtner ${ }^{114}$, D. Schaefer ${ }^{37}$, L. Schaefer ${ }^{137}$, J. Schaeffer ${ }^{100}$, S. Schaepe ${ }^{36}$, U. Schäfer ${ }^{100}$, A.C. Schaffer ${ }^{65}$, D. Schaile ${ }^{114}$, R.D. Schamberger ${ }^{155}$, N. Scharmberg ${ }^{101}$, V.A. Schegelsky ${ }^{138}$, D. Scheirich ${ }^{143}$, F. Schenck ${ }^{19}$, M. Schernau ${ }^{171}$, C. Schiavi ${ }^{55 b, 55 a}$, S. Schier ${ }^{146}$, L.K. Schildgen ${ }^{24}$, Z.M. Schillaci ${ }^{26}$, E.J. Schioppa ${ }^{36}$, M. Schioppa ${ }^{41 b, 41 a}$, K.E. Schleicher ${ }^{52}$, S. Schlenker ${ }^{36}$, K.R. Schmidt-Sommerfeld ${ }^{115}$, K. Schmieden ${ }^{36}$, C. Schmitt ${ }^{100}$, S. Schmitt ${ }^{46}$, S. Schmitz ${ }^{100}$, J.C. Schmoeckel ${ }^{46}$, U. Schnoor ${ }^{52}$, L. Schoeffel ${ }^{145}$, A. Schoening ${ }^{61 b}$, P.G. Scholer ${ }^{52}$, E. Schopf ${ }^{135}$, M. Schott ${ }^{100}$, J.F.P. Schouwenberg ${ }^{119}$, J. Schovancova ${ }^{36}$, S. Schramm ${ }^{54}$, F. Schroeder ${ }^{182}$, A. Schulte ${ }^{100}$, H-C. Schultz-Coulon ${ }^{61 a}$, M. Schumacher ${ }^{52}$, B.A. Schumm ${ }^{146}$, Ph. Schune ${ }^{145}$, A. Schwartzman ${ }^{153}$, T.A. Schwarz ${ }^{106}$, Ph. Schwemling ${ }^{145}$, R. Schwienhorst ${ }^{107}$, A. Sciandra ${ }^{146}$, G. Sciolla ${ }^{26}$, M. Scodeggio ${ }^{46}$, M. Scornajenghi ${ }^{41 b, 41 a}$, F. Scuri ${ }^{72 a}$, F. Scutti ${ }^{105}$, L.M. Scyboz ${ }^{115}$, C.D. Sebastiani ${ }^{73 a, 73 b}$, P. Seema ${ }^{19}$, S.C. Seidel ${ }^{118}$, A. Seiden ${ }^{146}$, B.D. Seidlitz ${ }^{29}$, T. Seiss ${ }^{37}$, J.M. Seixas ${ }^{81 b}$, G. Sekhniaidze ${ }^{70 a}$, K. Sekhon ${ }^{106}$, S.J. Sekula ${ }^{42}$, N. Semprini-Cesari ${ }^{23 b, 23 a,}$ S. Sen ${ }^{49}$, S. Senkin ${ }^{38}$, C. Serfon ${ }^{77}$, L. Serin ${ }^{65}$, L. Serkin ${ }^{67 a, 67 b}$, M. Sessa ${ }^{60 a}$, H. Severini ${ }^{129}$, T. Śfiligoj ${ }^{92}$, F. Sforza ${ }^{55 b, 55 a}$, A. Sfyrla ${ }^{54}$, E. Shabalina ${ }^{53}$, J.D. Shahinian ${ }^{146}$, N.W. Shaikh ${ }^{45 a, 45 b}$, D. Shaked Renous ${ }^{180}$, L.Y. Shan ${ }^{15 a}$, R. Shang ${ }^{173}$, J.T. Shank ${ }^{25}$, M. Shapiro ${ }^{18}$, A. Sharma ${ }^{135}$, A.S. Sharma ${ }^{1}$, P.B. Shatalov ${ }^{124}$, K. Shaw ${ }^{156}$, S.M. Shaw ${ }^{101}$, A. Shcherbakova ${ }^{138}$, M. Shehade ${ }^{180}$, Y. Shen ${ }^{129}$, N. Sherafati ${ }^{34}$, A.D. Sherman ${ }^{25}$, P. Sherwood ${ }^{95}$, L. Shi ${ }^{158, a u}$, S. Shimizu ${ }^{82}$, C.O. Shimmin ${ }^{183}$, Y. Shimogama ${ }^{179}$, M. Shimojima ${ }^{116}$, I.P.J. Shipsey ${ }^{135}$, S. Shirabe ${ }^{88}$, M. Shiyakova ${ }^{80, \text { ad }}$, J. Shlomi ${ }^{180}$, A. Shmeleva ${ }^{111}$, M.J. Shochet ${ }^{37}$, J. Shojaii ${ }^{105}$, D.R. Shope ${ }^{129}$, S. Shrestha ${ }^{127}$, E.M. Shrif ${ }^{33 e}$, E. Shulga ${ }^{180}$, P. Sicho ${ }^{141}$, A.M. Sickles ${ }^{173}$, P.E. Sidebo ${ }^{154}$, E. Sideras Haddad ${ }^{33 e}$, O. Sidiropoulou ${ }^{36}$, A. Sidoti2 ${ }^{23 b, 23 a}$, F. Siegert ${ }^{48}$, Dj. Sijacki ${ }^{16}$, M.Jr. Silva ${ }^{181}$, M.V. Silva Oliveira ${ }^{81 a}$, S.B. Silverstein ${ }^{45 a}$, S. Simion ${ }^{65}$, E. Simioni ${ }^{100}$, R. Simoniello ${ }^{100}$, S. Simsek ${ }^{12 b}$, P. Sinervo ${ }^{167}$, V. Sinetckii ${ }^{113,111}$, N.B. Sinev ${ }^{132}$, M. Sioli ${ }^{23 b, 23 a}$, I. Siral ${ }^{106}$, S.Yu. Sivoklokov ${ }^{113}$, J. Sjölin ${ }^{45 a, 45 b}$, E. Skorda ${ }^{97}$, P. Skubic ${ }^{129}$, M. Slawinska ${ }^{85}$, K. Sliwa ${ }^{170}$, R. Slovak ${ }^{143}$, V. Smakhtin ${ }^{180}$, B.H. Smart ${ }^{144}$, J. Smiesko ${ }^{28 a}$, N. Smirnov ${ }^{112}$, S.Yu. Smirnov ${ }^{112}$, Y. Smirnov ${ }^{112}$, L.N. Smirnova ${ }^{113, v}$, O. Smirnova ${ }^{97}$, J.W. Smith ${ }^{53}$, M. Smizanska ${ }^{90}$, K. Smolek ${ }^{142}$, A. Smykiewicz ${ }^{85}$, A.A. Snesarev ${ }^{111}$, H.L. Snoek ${ }^{120}$, I.M. Snyder ${ }^{132}$, S. Snyder ${ }^{29}$, R. Sobie ${ }^{176, \text { af }}$, A. Soffer ${ }^{161}$, A. Søgaard ${ }^{50}$, F. Sohns ${ }^{53}$, C.A. Solans Sanchez ${ }^{36}$, E.Yu. Soldatov ${ }^{112}$, U. Soldevila ${ }^{174}$, A.A. Solodkov ${ }^{123}$, A. Soloshenko ${ }^{80}$, O.V. Solovyanov ${ }^{123}$, V. Solovyev ${ }^{138}$, P. Sommer ${ }^{149}$, H. Son ${ }^{170}$, W. Song ${ }^{144}$, W.Y. Song ${ }^{168 b}$, A. Sopczak ${ }^{142}$, F. Sopkova ${ }^{28 b}$, C.L. Sotiropoulou ${ }^{72,72 b}$, S. Sottocornola ${ }^{71 a, 71 b}$, R. Soualah ${ }^{67 a, 67 c, g}$, A.M. Soukharev ${ }^{122 \mathrm{~b}, 122 \mathrm{a}}$, D. South ${ }^{46}$, S. Spagnolo ${ }^{68 \mathrm{a}, 68 \mathrm{~b}}$, M. Spalla ${ }^{115}$, M. Spangenberg ${ }^{178}$, F. Spanò ${ }^{94}$, D. Sperlich ${ }^{52}$, T.M. Spieker ${ }^{61 a}$, R. Spighi ${ }^{23 b}$, G. Spigo ${ }^{36}$, M. Spina ${ }^{156}$, D.P. Spiteri ${ }^{57}$, M. Spousta ${ }^{143}$, A. Stabile ${ }^{69 a, 69 b}$, B.L. Stamas ${ }^{121}$, R. Stamen $^{61 a}$, M. Stamenkovic ${ }^{120}$, 
E. Stanecka ${ }^{85}$, B. Stanislaus ${ }^{135}$, M.M. Stanitzki ${ }^{46}$, M. Stankaityte ${ }^{135}$, B. Stapf ${ }^{120}$, E.A. Starchenko ${ }^{123}$, G.H. Stark ${ }^{146}$, J. Stark ${ }^{58}$, S.H. Stark ${ }^{40}$, P. Staroba ${ }^{141}$, P. Starovoitov ${ }^{61 a}$, S. Stärz ${ }^{104}$, R. Staszewski ${ }^{85}$, G. Stavropoulos ${ }^{44}$, M. Stegler ${ }^{46}$, P. Steinberg ${ }^{29}$, A.L. Steinhebel ${ }^{132}$, B. Stelzer ${ }^{152}$, H.J. Stelzer ${ }^{139}$, O. Stelzer-Chilton ${ }^{168 a}$, H. Stenzel ${ }^{56}$, T.J. Stevenson ${ }^{156}$, G.A. Stewart ${ }^{36}$, M.C. Stockton ${ }^{36}$, G. Stoicea ${ }^{27 b}$, M. Stolarski ${ }^{140 a}$, S. Stonjek ${ }^{115}$, A. Straessner ${ }^{48}$, J. Strandberg ${ }^{154}$, S. Strandberg ${ }^{45 a, 45 b}$, M. Strauss ${ }^{129}$, P. Strizenec ${ }^{28 b}$, R. Ströhmer ${ }^{177}$, D.M. Strom ${ }^{132}$, R. Stroynowski ${ }^{42}$, A. Strubig $^{50}$, S.A. Stucci ${ }^{29}$, B. Stugu ${ }^{17}$, J. Stupak ${ }^{129}$, N.A. Styles ${ }^{46}$, D. $\mathrm{Su}^{153}$, S. Suchek ${ }^{61 \mathrm{a}}$, V.V. Sulin ${ }^{111}$, M.J. Sullivan ${ }^{91}$, D.M.S. Sultan ${ }^{54}$, S. Sultansoy ${ }^{4 c}$, T. Sumida ${ }^{86}$, S. Sun ${ }^{106}$, X. Sun ${ }^{3}$, K. Suruliz ${ }^{156}$, C.J.E. Suster ${ }^{157}$, M.R. Sutton ${ }^{156}$, S. Suzuki ${ }^{82}$, M. Svatos ${ }^{141}$, M. Swiatlowski ${ }^{37}$, S.P. Swift ${ }^{2}$, T. Swirski ${ }^{177}$, A. Sydorenko ${ }^{100}$,

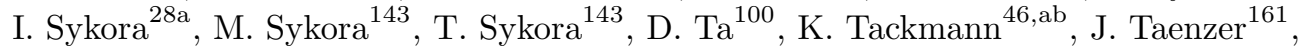

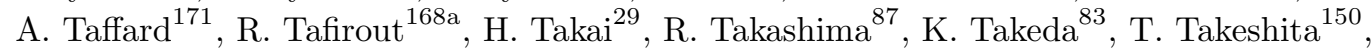
E.P. Takeva ${ }^{50}$, Y. Takubo ${ }^{82}$, M. Talby ${ }^{102}$, A.A. Talyshev ${ }^{122 b, 122 a}$, N.M. Tamir ${ }^{161}$, J. Tanaka ${ }^{163}$, M. Tanaka ${ }^{165}$, R. Tanaka ${ }^{65}$, S. Tapia Araya ${ }^{173}$, S. Tapprogge ${ }^{100}$,

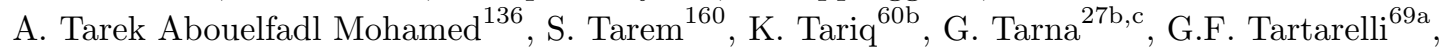
P. $\operatorname{Tas}^{143}$, M. Tasevsky ${ }^{141}$, T. Tashiro ${ }^{86}$, E. Tassi ${ }^{41 \mathrm{~b}, 41 \mathrm{a}}$, A. Tavares Delgado ${ }^{140 \mathrm{a}, 140 \mathrm{~b}}$, Y. Tayalati ${ }^{35 e}$, A.J. Taylor ${ }^{50}$, G.N. Taylor ${ }^{105}$, W. Taylor ${ }^{168 b}$, A.S. Tee ${ }^{90}$, R. Teixeira De Lima ${ }^{153}$, P. Teixeira-Dias ${ }^{94}$, H. Ten Kate ${ }^{36}$, J.J. Teoh ${ }^{120}$, S. Terada ${ }^{82}$, K. Terashi ${ }^{163}$, J. Terron ${ }^{99}$,

S. Terzo ${ }^{14}$, M. Testa ${ }^{51}$, R.J. Teuscher ${ }^{167, \text { af }}$, S.J. Thais ${ }^{183}$, T. Theveneaux-Pelzer ${ }^{46}$, F. Thiele ${ }^{40}$, D.W. Thomas ${ }^{94}$, J.O. Thomas ${ }^{42}$, J.P. Thomas ${ }^{21}$, A.S. Thompson ${ }^{57}$, P.D. Thompson ${ }^{21}$, L.A. Thomsen ${ }^{183}$, E. Thomson ${ }^{137}$, E.J. Thorpe ${ }^{93}$, Y. Tian ${ }^{39}$, R.E. Ticse Torres ${ }^{53}$, V.O. Tikhomirov ${ }^{111, a q}$, Yu.A. Tikhonov ${ }^{122 b, 122 a}$, S. Timoshenko ${ }^{112}$, P. Tipton ${ }^{183}$, S. Tisserant ${ }^{102}$, K. Todome ${ }^{23 b, 23 a}$, S. Todorova-Nova ${ }^{5}$, S. Todt ${ }^{48}$, J. Tojo ${ }^{88}$, S. Tokár ${ }^{28 a}$, K. Tokushuku ${ }^{82}$, E. Tolley ${ }^{127}$, K.G. Tomiwa ${ }^{33 e}$, M. Tomoto ${ }^{117}$, L. Tompkins ${ }^{153, q}$, B. Tong ${ }^{59}$, P. Tornambe ${ }^{103}$ E. Torrence ${ }^{132}$, H. Torres ${ }^{48}$, E. Torró Pastor ${ }^{148}$, C. Tosciri ${ }^{135}$, J. Toth $^{102, \text { ae }}$, D.R. Tovey ${ }^{149}$,

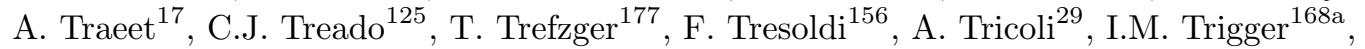
S. Trincaz-Duvoid ${ }^{136}$, W. Trischuk ${ }^{167}$, B. Trocmé ${ }^{58}$, A. Trofymov ${ }^{145}$, C. Troncon ${ }^{69 a}$,

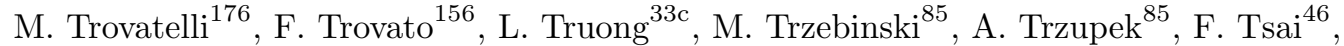
J.C-L. Tseng ${ }^{135}$, P.V. Tsiareshka ${ }^{108, a l}$, A. Tsirigotis ${ }^{162}$, N. Tsirintanis ${ }^{9}$, V. Tsiskaridze ${ }^{155}$, E.G. Tskhadadze ${ }^{159 a}$, M. Tsopoulou ${ }^{162}$, I.I. Tsukerman ${ }^{124}$, V. Tsulaia ${ }^{18}$, S. Tsuno ${ }^{82}$,

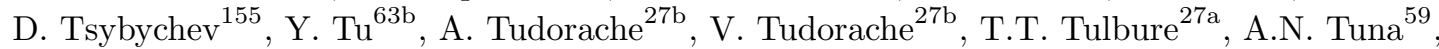
S. Turchikhin ${ }^{80}$, D. Turgeman ${ }^{180}$, I. Turk Cakir ${ }^{4 b, w}$, R.J. Turner ${ }^{21}$, R. Turra ${ }^{69 a}$, P.M. Tuts ${ }^{39}$,

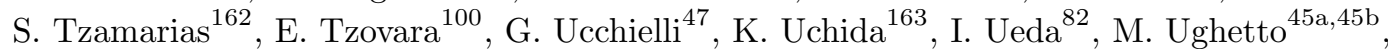

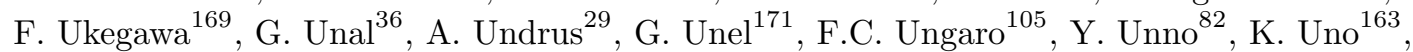

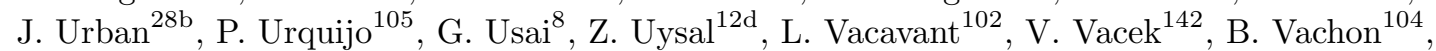
K.O.H. Vadla ${ }^{134}$, A. Vaidya ${ }^{95}$, C. Valderanis ${ }^{114}$, E. Valdes Santurio ${ }^{45 a, 45 b}$, M. Valente ${ }^{54}$, S. Valentinetti ${ }^{23 b, 23 a}$, A. Valero ${ }^{174}$, L. Valéry ${ }^{46}$, R.A. Vallance ${ }^{21}$, A. Vallier ${ }^{36}$, J.A. Valls Ferrer ${ }^{174}$, T.R. Van Daalen ${ }^{14}$, P. Van Gemmeren ${ }^{6}$, I. Van Vulpen ${ }^{120}$, M. Vanadia ${ }^{74 a, 74 b}$, W. Vandelli ${ }^{36}$,

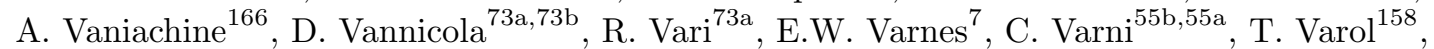
D. Varouchas ${ }^{65}$, K.E. Varvell ${ }^{157}$, M.E. Vasile ${ }^{27 b}$, G.A. Vasquez ${ }^{176}$, J.G. Vasquez ${ }^{183}$, F. Vazeille ${ }^{38}$, D. Vazquez Furelos ${ }^{14}$, T. Vazquez Schroeder ${ }^{36}$, J. Veatch ${ }^{53}$, V. Vecchio ${ }^{75 a, 75 b}$, M.J. Veen ${ }^{120}$, L.M. Veloce ${ }^{167}$, F. Veloso ${ }^{140 a, 140 c}$, S. Veneziano ${ }^{73 a}$, A. Ventura ${ }^{68 a, 68 b}$, N. Venturi $^{36}$,

A. Verbytskyi ${ }^{115}$, V. Vercesi ${ }^{71 a}$, M. Verducci ${ }^{72 a, 72 b}$, C.M. Vergel Infante ${ }^{79}$, C. Vergis ${ }^{24}$, W. Verkerke ${ }^{120}$, A.T. Vermeulen ${ }^{120}$, J.C. Vermeulen ${ }^{120}$, M.C. Vetterli ${ }^{152, \text { ax }}$, N. Viaux Maira ${ }^{147 d}$, M. Vicente Barreto Pinto ${ }^{54}$, T. Vickey ${ }^{149}$, O.E. Vickey Boeriu ${ }^{149}$, G.H.A. Viehhauser ${ }^{135}$,

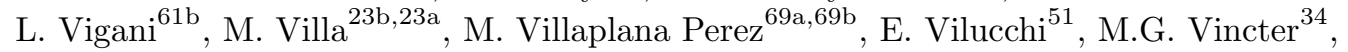
G.S. Virdee ${ }^{21}$, A. Vishwakarma ${ }^{46}$, C. Vittori ${ }^{23 b, 23 a}$, I. Vivarelli $^{156}$, M. Vogel $^{182}$, P. $_{\text {Vokac }}{ }^{142}$, S.E. von Buddenbrock ${ }^{33 e}$, E. Von Toerne ${ }^{24}$, V. Vorobel $^{143}$, K. Vorobev $^{112}$, M. Vos ${ }^{174}$, 
J.H. Vossebeld ${ }^{91}$, M. Vozak ${ }^{101}$, N. Vranjes ${ }^{16}$, M. Vranjes Milosavljevic ${ }^{16}$, V. Vrba ${ }^{142}$, M. Vreeswijk ${ }^{120}$, R. Vuillermet ${ }^{36}$, I. Vukotic ${ }^{37}$, P. Wagner ${ }^{24}$, W. Wagner ${ }^{182}$, J. Wagner-Kuhr ${ }^{114}$, S. Wahdan ${ }^{182}$, H. Wahlberg ${ }^{89}$, V.M. Walbrecht ${ }^{115}$, J. Walder ${ }^{90}$, R. Walker ${ }^{114}$, S.D. Walker ${ }^{94}$,

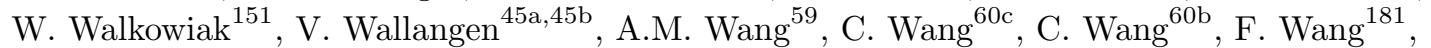

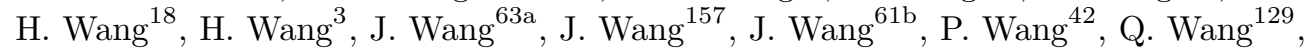
R.-J. Wang ${ }^{100}$, R. Wang ${ }^{60 a}$, R. Wang ${ }^{6}$, S.M. Wang ${ }^{158}$, W.T. Wang ${ }^{60 a}$, W. Wang ${ }^{15 c, a g}$, W.X. Wang ${ }^{60 a, a g}$, Y. Wang ${ }^{60 a, a n}$, Z. Wang ${ }^{60 c}$, C. Wanotayaroj ${ }^{46}$, A. Warburton ${ }^{104}$, C.P. Ward ${ }^{32}$, D.R. Wardrope ${ }^{95}$, N. Warrack ${ }^{57}$, A. Washbrook ${ }^{50}$, A.T. Watson ${ }^{21}$, M.F. Watson ${ }^{21}$, G. Watts ${ }^{148}$, B.M. Waugh ${ }^{95}$, A.F. Webb ${ }^{11}$, S. Webb ${ }^{100}$, C. Weber ${ }^{183}$, M.S. Weber ${ }^{20}$, S.A. Weber ${ }^{34}$, S.M. Weber ${ }^{61 a}$, A.R. Weidberg ${ }^{135}$, J. Weingarten ${ }^{47}$, M. Weirich ${ }^{100}$, C. Weiser ${ }^{52}$, P.S. Wells ${ }^{36}$, T. Wenaus ${ }^{29}$, T. Wengler ${ }^{36}$, S. Wenig ${ }^{36}$, N. Wermes ${ }^{24}$, M.D. Werner ${ }^{79}$, M. Wessels $^{61 a}$, T.D. Weston ${ }^{20}$, K. Whalen ${ }^{132}$, N.L. Whallon ${ }^{148}$, A.M. Wharton ${ }^{90}$, A.S. White ${ }^{106}$, A. White ${ }^{8}$, M.J. White ${ }^{1}$, D. Whiteson ${ }^{171}$, B.W. Whitmore ${ }^{90}$, W. Wiedenmann ${ }^{181}$, M. Wielers ${ }^{144}$, N. Wieseotte ${ }^{100}$, C. Wiglesworth ${ }^{40}$, L.A.M. Wiik-Fuchs ${ }^{52}$, F. Wilk ${ }^{101}$, H.G. Wilkens ${ }^{36}$, L.J. Wilkins ${ }^{94}$, H.H. Williams ${ }^{137}$, S. Williams ${ }^{32}$, C. Willis ${ }^{107}$, S. Willocq ${ }^{103}$, J.A. Wilson ${ }^{21}$, I. Wingerter-Seez ${ }^{5}$, E. Winkels ${ }^{156}$, F. Winklmeier ${ }^{132}$, O.J. Winston ${ }^{156}$, B.T. Winter ${ }^{52}$, M. Wittgen ${ }^{153}$, M. Wobisch ${ }^{96}$, A. Wolf ${ }^{100}$, T.M.H. Wolf ${ }^{120}$, R. Wolff ${ }^{102}$, R. Wölker ${ }^{135}$, J. Wollrath ${ }^{52}$, M.W. Wolter ${ }^{85}$, H. Wolters ${ }^{140 a, 140 c}$, V.W.S. Wong ${ }^{175}$, N.L. Woods ${ }^{146}$, S.D. Worm ${ }^{21}$, B.K. Wosiek ${ }^{85}$, K.W. Woźniak ${ }^{85}$, K. Wraight ${ }^{57}$, S.L. Wu ${ }^{181}$, X. Wu ${ }^{54}$, Y. Wu ${ }^{60 a}$, T.R. Wyatt ${ }^{101}$, B.M. Wynne ${ }^{50}$, S. Xella ${ }^{40}$, Z. Xi ${ }^{106}$, L. Xia $^{178}$, X. Xiao ${ }^{106}$, I. Xiotidis ${ }^{156}$, D. $\mathrm{Xu}^{15 \mathrm{a}}$, H. Xu ${ }^{60 \mathrm{a}, \mathrm{c}}$, L. $\mathrm{Xu}^{29}$, T. $\mathrm{Xu}^{145}$, W. Xu ${ }^{106}$, Z. Xu ${ }^{60 \mathrm{~b}}$, Z. Xu ${ }^{153}$, B. Yabsley ${ }^{157}$, S. Yacoob ${ }^{33 a}$, K. Yajima ${ }^{133}$, D.P. Yallup ${ }^{95}$, D. Yamaguchi ${ }^{165}$, Y. Yamaguchi ${ }^{165}$, A. Yamamoto ${ }^{82}$, M. Yamatani ${ }^{163}$, T. Yamazaki ${ }^{163}$, Y. Yamazaki ${ }^{83}$, Z. Yan ${ }^{25}$, H.J. Yang ${ }^{60 c, 60 d}$, H.T. Yang ${ }^{18}$, S. Yang ${ }^{78}$, X. Yang ${ }^{60 b, 58}$, Y. Yang ${ }^{163}$, W-M. Yao ${ }^{18}$, Y.C. Yap ${ }^{46}$, Y. Yasu ${ }^{82}$, E. Yatsenko ${ }^{60 c, 60 d}$, J. Ye ${ }^{42}$, S. Ye ${ }^{29}$, I. Yeletskikh ${ }^{80}$, M.R. Yexley ${ }^{90}$, E. Yigitbasi ${ }^{25}$, K. Yorita ${ }^{179}$, K. Yoshihara ${ }^{137}$, C.J.S. Young ${ }^{36}$, C. Young ${ }^{153}$, J. Yu ${ }^{79}$, R. Yuan ${ }^{60 b, i}$, X. Yue ${ }^{61 a}$, S.P.Y. Yuen ${ }^{24}$, M. Zaazoua ${ }^{35 e}$, B. Zabinski ${ }^{85}$, G. Zacharis ${ }^{10}$, E. Zaffaroni ${ }^{54}$, J. Zahreddine ${ }^{136}$, A.M. Zaitsev ${ }^{123, \text { ap }}$,

T. Zakareishvili ${ }^{159 b}$, N. Zakharchuk ${ }^{34}$, S. Zambito ${ }^{59}$, D. Zanzi ${ }^{36}$, D.R. Zaripovas ${ }^{57}$, S.V. Zeißner ${ }^{47}$, C. Zeitnitz ${ }^{182}$, G. Zemaityte ${ }^{135}$, J.C. Zeng ${ }^{173}$, O. Zenin ${ }^{123}$, T. Ženišs ${ }^{28 a}$, D. Zerwas ${ }^{65}$,

M. Zgubic ${ }^{135}$, D.F. Zhang ${ }^{15 b}$, G. Zhang ${ }^{15 b}$, H. Zhang ${ }^{15 c}$, J. Zhang ${ }^{6}$, L. Zhang ${ }^{15 c}$, L. Zhang ${ }^{60 a}$, M. Zhang ${ }^{173}$, R. Zhang ${ }^{24}$, X. Zhang ${ }^{60 b}$, Y. Zhang ${ }^{15 a, 15 d}$, Z. Zhang ${ }^{63 a}$, Z. Zhang ${ }^{65}$, P. Zhao ${ }^{49}$, Y. Zhao ${ }^{60 \mathrm{~b}}$, Z. Zhao ${ }^{60 \mathrm{a}}$, A. Zhemchugov ${ }^{80}$, Z. Zheng ${ }^{106}$, D. Zhong ${ }^{173}$, B. Zhou ${ }^{106}$, C. Zhou ${ }^{181}$, M.S. Zhou ${ }^{15 a, 15 d}$, M. Zhou ${ }^{155}$, N. Zhou ${ }^{60 c}$, Y. Zhou ${ }^{7}$, C.G. Zhu ${ }^{60 b}$, H.L. Zhu ${ }^{60 a}$, H. Zhu ${ }^{15 a}$, J. Zhu ${ }^{106}$, Y. Zhu ${ }^{60 a}$, X. Zhuang ${ }^{15 a}$, K. Zhukov ${ }^{111}$, V. Zhulanov ${ }^{122 b, 122 a}$, D. Zieminska ${ }^{66}$, N.I. Zimine ${ }^{80}$, S. Zimmermann ${ }^{52}$, Z. Zinonos ${ }^{115}$, M. Ziolkowski ${ }^{151}$, L. Živković ${ }^{16}$, G. Zobernig ${ }^{181}$, A. Zoccoli ${ }^{23 b, 23 a}$, K. Zoch ${ }^{53}$, T.G. Zorbas ${ }^{149}$, R. Zou ${ }^{37}$, L. Zwalinski ${ }^{36}$

1 Department of Physics, University of Adelaide, Adelaide, Australia

2 Physics Department, SUNY Albany, Albany NY, United States of America

3 Department of Physics, University of Alberta, Edmonton AB, Canada

4 (a) Department of Physics, Ankara University, Ankara; ${ }^{(b)}$ Istanbul Aydin University, Application and Research Center for Advanced Studies, Istanbul; ${ }^{(c)}$ Division of Physics, TOBB University of Economics and Technology, Ankara, Turkey

5 LAPP, Université Grenoble Alpes, Université Savoie Mont Blanc, CNRS/IN2P3, Annecy, France

6 High Energy Physics Division, Argonne National Laboratory, Argonne IL, United States of America

7 Department of Physics, University of Arizona, Tucson AZ, United States of America

8 Department of Physics, University of Texas at Arlington, Arlington TX, United States of America

9 Physics Department, National and Kapodistrian University of Athens, Athens, Greece

10 Physics Department, National Technical University of Athens, Zografou, Greece 
$12{ }^{(a)}$ Bahcesehir University, Faculty of Engineering and Natural Sciences, Istanbul; ${ }^{(b)}$ Istanbul Bilgi University, Faculty of Engineering and Natural Sciences, Istanbul; ${ }^{(c)}$ Department of Physics, Bogazici University, Istanbul; ${ }^{(d)}$ Department of Physics Engineering, Gaziantep University, Gaziantep, Turkey

13 Institute of Physics, Azerbaijan Academy of Sciences, Baku, Azerbaijan

14 Institut de Física d'Altes Energies (IFAE), Barcelona Institute of Science and Technology, Barcelona, Spain

15 (a) Institute of High Energy Physics, Chinese Academy of Sciences, Beijing; ${ }^{(b)}$ Physics Department, Tsinghua University, Beijing; ${ }^{\left({ }^{c}\right)}$ Department of Physics, Nanjing University, Nanjing; ${ }^{(d)}$ University of Chinese Academy of Science (UCAS), Beijing, China

16 Institute of Physics, University of Belgrade, Belgrade, Serbia

${ }_{17}$ Department for Physics and Technology, University of Bergen, Bergen, Norway

18 Physics Division, Lawrence Berkeley National Laboratory and University of California, Berkeley CA, United States of America

19 Institut für Physik, Humboldt Universität zu Berlin, Berlin, Germany

20 Albert Einstein Center for Fundamental Physics and Laboratory for High Energy Physics, University of Bern, Bern, Switzerland

${ }^{21}$ School of Physics and Astronomy, University of Birmingham, Birmingham, United Kingdom

${ }^{22}{ }^{(a)}$ Facultad de Ciencias y Centro de Investigaciónes, Universidad Antonio Nariño, Bogotá;

${ }^{(b)}$ Departamento de Física, Universidad Nacional de Colombia, Bogotá, Colombia, Colombia

${ }^{23}{ }^{(a)}$ INFN Bologna and Universita' di Bologna, Dipartimento di Fisica; ${ }^{(b)}$ INFN Sezione di Bologna, Italy

24 Physikalisches Institut, Universität Bonn, Bonn, Germany

25 Department of Physics, Boston University, Boston MA, United States of America

26 Department of Physics, Brandeis University, Waltham MA, United States of America

${ }^{27}{ }^{(a)}$ Transilvania University of Brasov, Brasov; ${ }^{(b)}$ Horia Hulubei National Institute of Physics and Nuclear Engineering, Bucharest; ${ }^{\left({ }^{c}\right)}$ Department of Physics, Alexandru Ioan Cuza University of Iasi, Iasi; ${ }^{(d)}$ National Institute for Research and Development of Isotopic and Molecular Technologies, Physics Department, Cluj-Napoca; ${ }^{(e)}$ University Politehnica Bucharest, Bucharest; ${ }^{(f)}$ West University in Timisoara, Timisoara, Romania

${ }^{28}{ }^{(a)}$ Faculty of Mathematics, Physics and Informatics, Comenius University, Bratislava;

(b) Department of Subnuclear Physics, Institute of Experimental Physics of the Slovak Academy of Sciences, Kosice, Slovak Republic

29 Physics Department, Brookhaven National Laboratory, Upton NY, United States of America

30 Departamento de Física, Universidad de Buenos Aires, Buenos Aires, Argentina

31 California State University, CA, United States of America

32 Cavendish Laboratory, University of Cambridge, Cambridge, United Kingdom

${ }^{33}{ }^{(a)}$ Department of Physics, University of Cape Town, Cape Town; ${ }^{\left({ }^{b}\right)}$ iThemba Labs, Western Cape;

(c) Department of Mechanical Engineering Science, University of Johannesburg, Johannesburg;

${ }^{(d)}$ University of South Africa, Department of Physics, Pretoria; ${ }^{(e)}$ School of Physics, University of the Witwatersrand, Johannesburg, South Africa

34 Department of Physics, Carleton University, Ottawa ON, Canada

${ }^{35}{ }^{(a)}$ Faculté des Sciences Ain Chock, Réseau Universitaire de Physique des Hautes Energies Université Hassan II, Casablanca; ${ }^{\left({ }^{b}\right)}$ Faculté des Sciences, Université Ibn-Tofail, Kénitra;

${ }^{(c)}$ Faculté des Sciences Semlalia, Université Cadi Ayyad, LPHEA-Marrakech; ${ }^{(d)}$ Faculté des Sciences, Université Mohamed Premier and LPTPM, Oujda; ${ }^{(e)}$ Faculté des sciences, Université Mohammed V, Rabat, Morocco

${ }^{36}$ CERN, Geneva, Switzerland

37 Enrico Fermi Institute, University of Chicago, Chicago IL, United States of America

38 LPC, Université Clermont Auvergne, CNRS/IN2P3, Clermont-Ferrand, France

39 Nevis Laboratory, Columbia University, Irvington NY, United States of America 
Niels Bohr Institute, University of Copenhagen, Copenhagen, Denmark

$41{ }^{(a)}$ Dipartimento di Fisica, Università della Calabria, Rende; ${ }^{(b)}$ INFN Gruppo Collegato di Cosenza, Laboratori Nazionali di Frascati, Italy

42 Physics Department, Southern Methodist University, Dallas TX, United States of America

43 Physics Department, University of Texas at Dallas, Richardson TX, United States of America

44 National Centre for Scientific Research "Demokritos", Agia Paraskevi, Greece

$45{ }^{(a)}$ Department of Physics, Stockholm University; ${ }^{(b)}$ Oskar Klein Centre, Stockholm, Sweden

46 Deutsches Elektronen-Synchrotron DESY, Hamburg and Zeuthen, Germany

47 Lehrstuhl für Experimentelle Physik IV, Technische Universität Dortmund, Dortmund, Germany

48 Institut für Kern und Teilchenphysik, Technische Universität Dresden, Dresden, Germany

49 Department of Physics, Duke University, Durham NC, United States of America

50 SUPA - School of Physics and Astronomy, University of Edinburgh, Edinburgh, United Kingdom

51 INFN e Laboratori Nazionali di Frascati, Frascati, Italy

52 Physikalisches Institut, Albert-Ludwigs-Universität Freiburg, Freiburg, Germany

53 II. Physikalisches Institut, Georg-August-Universität Göttingen, Göttingen, Germany

54 Département de Physique Nucléaire et Corpusculaire, Université de Genève, Genève, Switzerland

$55{ }^{(a)}$ Dipartimento di Fisica, Università di Genova, Genova; ${ }^{(b)}$ INFN Sezione di Genova, Italy

56 II. Physikalisches Institut, Justus-Liebig-Universität Giessen, Giessen, Germany

57 SUPA - School of Physics and Astronomy, University of Glasgow, Glasgow, United Kingdom

58 LPSC, Université Grenoble Alpes, CNRS/IN2P3, Grenoble INP, Grenoble, France

59 Laboratory for Particle Physics and Cosmology, Harvard University, Cambridge MA, United States of America

$60{ }^{(a)}$ Department of Modern Physics and State Key Laboratory of Particle Detection and Electronics, University of Science and Technology of China, Hefei; ${ }^{(b)}$ Institute of Frontier and Interdisciplinary Science and Key Laboratory of Particle Physics and Particle Irradiation (MOE), Shandong University, Qingdao; ${ }^{\left({ }^{c}\right)}$ School of Physics and Astronomy, Shanghai Jiao Tong University, KLPPAC-MoE, SKLPPC, Shanghai; ${ }^{(d)}$ Tsung-Dao Lee Institute, Shanghai, China

$61{ }^{(a)}$ Kirchhoff-Institut für Physik, Ruprecht-Karls-Universität Heidelberg, Heidelberg;

${ }^{(b)}$ Physikalisches Institut, Ruprecht-Karls-Universität Heidelberg, Heidelberg, Germany

62 Faculty of Applied Information Science, Hiroshima Institute of Technology, Hiroshima, Japan

63 (a) Department of Physics, Chinese University of Hong Kong, Shatin, N.T., Hong Kong;

${ }^{(b)}$ Department of Physics, University of Hong Kong, Hong Kong; ${ }^{(c)}$ Department of Physics and Institute for Advanced Study, Hong Kong University of Science and Technology, Clear Water Bay, Kowloon, Hong Kong, China

64 Department of Physics, National Tsing Hua University, Hsinchu, Taiwan

65 IJCLab, Université Paris-Saclay, CNRS/IN2P3, 91405, Orsay, France

66 Department of Physics, Indiana University, Bloomington IN, United States of America

67 (a) INFN Gruppo Collegato di Udine, Sezione di Trieste, Udine; ${ }^{(b)}$ ICTP, Trieste; ${ }^{(c)}$ Dipartimento Politecnico di Ingegneria e Architettura, Università di Udine, Udine, Italy

$68{ }^{(a)}$ INFN Sezione di Lecce; ${ }^{(b)}$ Dipartimento di Matematica e Fisica, Università del Salento, Lecce, Italy

$69{ }^{(a)}$ INFN Sezione di Milano; ${ }^{(b)}$ Dipartimento di Fisica, Università di Milano, Milano, Italy

$70{ }^{(a)}$ INFN Sezione di Napoli; ${ }^{(b)}$ Dipartimento di Fisica, Università di Napoli, Napoli, Italy

71 (a) INFN Sezione di Pavia; ${ }^{(b)}$ Dipartimento di Fisica, Università di Pavia, Pavia, Italy

72 (a) INFN Sezione di Pisa; ${ }^{(b)}$ Dipartimento di Fisica E. Fermi, Università di Pisa, Pisa, Italy

73 (a) INFN Sezione di Roma; ${ }^{(b)}$ Dipartimento di Fisica, Sapienza Università di Roma, Roma, Italy

74 (a) INFN Sezione di Roma Tor Vergata; ${ }^{(b)}$ Dipartimento di Fisica, Università di Roma Tor Vergata, Roma, Italy

75 (a) INFN Sezione di Roma Tre; ${ }^{(b)}$ Dipartimento di Matematica e Fisica, Università Roma Tre, Roma, Italy

$76{ }^{(a)}$ INFN-TIFPA; ${ }^{(b)}$ Università degli Studi di Trento, Trento, Italy

77 Institut für Astro und Teilchenphysik, Leopold-Franzens-Universität, Innsbruck, Austria 
$81{ }^{(a)}$ Departamento de Engenharia Elétrica, Universidade Federal de Juiz de Fora (UFJF), Juiz de Fora; ${ }^{(b)}$ Universidade Federal do Rio De Janeiro COPPE/EE/IF, Rio de Janeiro; ${ }^{(c)}$ Universidade Federal de São João del Rei (UFSJ), São João del Rei; ${ }^{\left({ }^{(}\right)}$Instituto de Física, Universidade de São Paulo, São Paulo, Brazil

82 KEK, High Energy Accelerator Research Organization, Tsukuba, Japan

83 Graduate School of Science, Kobe University, Kobe, Japan

$84{ }^{(a)}$ AGH University of Science and Technology, Faculty of Physics and Applied Computer Science, Krakow; ${ }^{(b)}$ Marian Smoluchowski Institute of Physics, Jagiellonian University, Krakow, Poland

85 Institute of Nuclear Physics Polish Academy of Sciences, Krakow, Poland

86 Faculty of Science, Kyoto University, Kyoto, Japan

87 Kyoto University of Education, Kyoto, Japan

88 Research Center for Advanced Particle Physics and Department of Physics, Kyushu University, Fukuoka , Japan

89 Instituto de Física La Plata, Universidad Nacional de La Plata and CONICET, La Plata, Argentina

90 Physics Department, Lancaster University, Lancaster, United Kingdom

91 Oliver Lodge Laboratory, University of Liverpool, Liverpool, United Kingdom

92 Department of Experimental Particle Physics, Jožef Stefan Institute and Department of Physics, University of Ljubljana, Ljubljana, Slovenia

93 School of Physics and Astronomy, Queen Mary University of London, London, United Kingdom

94 Department of Physics, Royal Holloway University of London, Egham, United Kingdom

95 Department of Physics and Astronomy, University College London, London, United Kingdom

96 Louisiana Tech University, Ruston LA, United States of America

97 Fysiska institutionen, Lunds universitet, Lund, Sweden

98 Centre de Calcul de l'Institut National de Physique Nucléaire et de Physique des Particules (IN2P3), Villeurbanne, France

99 Departamento de Física Teorica C-15 and CIAFF, Universidad Autónoma de Madrid, Madrid, Spain

100 Institut für Physik, Universität Mainz, Mainz, Germany

101 School of Physics and Astronomy, University of Manchester, Manchester, United Kingdom

102 CPPM, Aix-Marseille Université, CNRS/IN2P3, Marseille, France

103 Department of Physics, University of Massachusetts, Amherst MA, United States of America

104 Department of Physics, McGill University, Montreal QC, Canada

105 School of Physics, University of Melbourne, Victoria, Australia

106 Department of Physics, University of Michigan, Ann Arbor MI, United States of America

107 Department of Physics and Astronomy, Michigan State University, East Lansing MI, United States of America

108 B.I. Stepanov Institute of Physics, National Academy of Sciences of Belarus, Minsk, Belarus

109 Research Institute for Nuclear Problems of Byelorussian State University, Minsk, Belarus

110 Group of Particle Physics, University of Montreal, Montreal QC, Canada

111 P.N. Lebedev Physical Institute of the Russian Academy of Sciences, Moscow, Russia

112 National Research Nuclear University MEPhI, Moscow, Russia

113 D. V. Skobeltsyn Institute of Nuclear Physics, M.V. Lomonosov Moscow State University, Moscow, Russia

114 Fakultät für Physik, Ludwig-Maximilians-Universität München, München, Germany

115 Max-Planck-Institut für Physik (Werner-Heisenberg-Institut), München, Germany

116 Nagasaki Institute of Applied Science, Nagasaki, Japan

117 Graduate School of Science and Kobayashi-Maskawa Institute, Nagoya University, Nagoya, Japan

118 Department of Physics and Astronomy, University of New Mexico, Albuquerque NM, United States of America 
120 Nikhef National Institute for Subatomic Physics and University of Amsterdam, Amsterdam, Netherlands

121 Department of Physics, Northern Illinois University, DeKalb IL, United States of America

$122{ }^{(a)}$ Budker Institute of Nuclear Physics and NSU, SB RAS, Novosibirsk; ${ }^{(b)}$ Novosibirsk State University Novosibirsk, Russia

123 Institute for High Energy Physics of the National Research Centre Kurchatov Institute, Protvino, Russia

124 Institute for Theoretical and Experimental Physics named by A.I. Alikhanov of National Research Centre "Kurchatov Institute", Moscow, Russia

125 Department of Physics, New York University, New York NY, United States of America

126 Ochanomizu University, Otsuka, Bunkyo-ku, Tokyo, Japan

127 Ohio State University, Columbus OH, United States of America

128 Faculty of Science, Okayama University, Okayama, Japan

129 Homer L. Dodge Department of Physics and Astronomy, University of Oklahoma, Norman OK, United States of America

130 Department of Physics, Oklahoma State University, Stillwater OK, United States of America

131 Palacký University, RCPTM, Joint Laboratory of Optics, Olomouc, Czech Republic

132 Institute for Fundamental Science, University of Oregon, Eugene, OR, United States of America

133 Graduate School of Science, Osaka University, Osaka, Japan

134 Department of Physics, University of Oslo, Oslo, Norway

135 Department of Physics, Oxford University, Oxford, United Kingdom

136 LPNHE, Sorbonne Université, Université de Paris, CNRS/IN2P3, Paris, France

137 Department of Physics, University of Pennsylvania, Philadelphia PA, United States of America

138 Konstantinov Nuclear Physics Institute of National Research Centre "Kurchatov Institute", PNPI, St. Petersburg, Russia

139 Department of Physics and Astronomy, University of Pittsburgh, Pittsburgh PA, United States of America

$140{ }^{(a)}$ Laboratório de Instrumentação e Física Experimental de Partículas - LIP, Lisboa;

${ }^{(b)}$ Departamento de Física, Faculdade de Ciências, Universidade de Lisboa, Lisboa;

${ }^{(c)}$ Departamento de Física, Universidade de Coimbra, Coimbra; ${ }^{(d)}$ Centro de Física Nuclear da Universidade de Lisboa, Lisboa; ${ }^{(e)}$ Departamento de Física, Universidade do Minho, Braga;

${ }^{(f)}$ Departamento de Física Teórica y del Cosmos, Universidad de Granada, Granada (Spain);

${ }^{(g)}$ Departamento de Física and CEFITEC of Faculdade de Ciências e Tecnologia, Universidade Nova de Lisboa, Caparica; ${ }^{(h)}$ Instituto Superior Técnico, Universidade de Lisboa, Lisboa, Portugal

141 Institute of Physics of the Czech Academy of Sciences, Prague, Czech Republic

142 Czech Technical University in Prague, Prague, Czech Republic

143 Charles University, Faculty of Mathematics and Physics, Prague, Czech Republic

144 Particle Physics Department, Rutherford Appleton Laboratory, Didcot, United Kingdom

145 IRFU, CEA, Université Paris-Saclay, Gif-sur-Yvette, France

146 Santa Cruz Institute for Particle Physics, University of California Santa Cruz, Santa Cruz CA, United States of America

$147{ }^{(a)}$ Departamento de Física, Pontificia Universidad Católica de Chile, Santiago; ${ }^{(b)}$ Universidad Andres Bello, Department of Physics, Santiago; ${ }^{(c)}$ Instituto de Alta Investigación, Universidad de Tarapacá; ${ }^{(d)}$ Departamento de Física, Universidad Técnica Federico Santa María, Valparaíso, Chile

148 Department of Physics, University of Washington, Seattle WA, United States of America

149 Department of Physics and Astronomy, University of Sheffield, Sheffield, United Kingdom

150 Department of Physics, Shinshu University, Nagano, Japan

151 Department Physik, Universität Siegen, Siegen, Germany

152 Department of Physics, Simon Fraser University, Burnaby BC, Canada

153 SLAC National Accelerator Laboratory, Stanford CA, United States of America 
Physics Department, Royal Institute of Technology, Stockholm, Sweden

Departments of Physics and Astronomy, Stony Brook University, Stony Brook NY, United States of America

Department of Physics and Astronomy, University of Sussex, Brighton, United Kingdom

157 School of Physics, University of Sydney, Sydney, Australia

158 Institute of Physics, Academia Sinica, Taipei, Taiwan

$159{ }^{(a)}$ E. Andronikashvili Institute of Physics, Iv. Javakhishvili Tbilisi State University, Tbilisi; ${ }^{(b)}$ High Energy Physics Institute, Tbilisi State University, Tbilisi, Georgia

160 Department of Physics, Technion, Israel Institute of Technology, Haifa, Israel

161 Raymond and Beverly Sackler School of Physics and Astronomy, Tel Aviv University, Tel Aviv, Israel

162 Department of Physics, Aristotle University of Thessaloniki, Thessaloniki, Greece

163 International Center for Elementary Particle Physics and Department of Physics, University of Tokyo, Tokyo, Japan

164 Graduate School of Science and Technology, Tokyo Metropolitan University, Tokyo, Japan

165 Department of Physics, Tokyo Institute of Technology, Tokyo, Japan

166 Tomsk State University, Tomsk, Russia

167 Department of Physics, University of Toronto, Toronto ON, Canada

$168{ }^{(a)}$ TRIUMF, Vancouver BC; ${ }^{(b)}$ Department of Physics and Astronomy, York University, Toronto ON, Canada

169 Division of Physics and Tomonaga Center for the History of the Universe, Faculty of Pure and Applied Sciences, University of Tsukuba, Tsukuba, Japan

170 Department of Physics and Astronomy, Tufts University, Medford MA, United States of America

171 Department of Physics and Astronomy, University of California Irvine, Irvine CA, United States of America

172 Department of Physics and Astronomy, University of Uppsala, Uppsala, Sweden

173 Department of Physics, University of Illinois, Urbana IL, United States of America

174 Instituto de Física Corpuscular (IFIC), Centro Mixto Universidad de Valencia - CSIC, Valencia, Spain

175 Department of Physics, University of British Columbia, Vancouver BC, Canada

176 Department of Physics and Astronomy, University of Victoria, Victoria BC, Canada

177 Fakultät für Physik und Astronomie, Julius-Maximilians-Universität Würzburg, Würzburg, Germany

178 Department of Physics, University of Warwick, Coventry, United Kingdom

179 Waseda University, Tokyo, Japan

180 Department of Particle Physics, Weizmann Institute of Science, Rehovot, Israel

181 Department of Physics, University of Wisconsin, Madison WI, United States of America

182 Fakultät für Mathematik und Naturwissenschaften, Fachgruppe Physik, Bergische Universität Wuppertal, Wuppertal, Germany

183 Department of Physics, Yale University, New Haven CT, United States of America

184 Yerevan Physics Institute, Yerevan, Armenia

a Also at Borough of Manhattan Community College, City University of New York, New York NY, United States of America

${ }^{b}$ Also at CERN, Geneva, Switzerland

c Also at CPPM, Aix-Marseille Université, CNRS/IN2P3, Marseille, France

${ }^{d}$ Also at Département de Physique Nucléaire et Corpusculaire, Université de Genève, Genève, Switzerland

e Also at Departament de Fisica de la Universitat Autonoma de Barcelona, Barcelona, Spain

${ }^{f}$ Also at Departamento de Física, Instituto Superior Técnico, Universidade de Lisboa, Lisboa, Portugal

$g$ Also at Department of Applied Physics and Astronomy, University of Sharjah, Sharjah, United Arab Emirates 
${ }^{h}$ Also at Department of Financial and Management Engineering, University of the Aegean, Chios, Greece

${ }^{i}$ Also at Department of Physics and Astronomy, Michigan State University, East Lansing MI, United States of America

${ }^{j}$ Also at Department of Physics and Astronomy, University of Louisville, Louisville, KY, United States of America

${ }^{k}$ Also at Department of Physics, Ben Gurion University of the Negev, Beer Sheva, Israel

${ }^{l}$ Also at Department of Physics, California State University, East Bay, United States of America

$m$ Also at Department of Physics, California State University, Fresno, United States of America

${ }^{n}$ Also at Department of Physics, California State University, Sacramento, United States of America

- Also at Department of Physics, King's College London, London, United Kingdom

${ }^{p}$ Also at Department of Physics, St. Petersburg State Polytechnical University, St. Petersburg, Russia

$q$ Also at Department of Physics, Stanford University, Stanford CA, United States of America

${ }^{r}$ Also at Department of Physics, University of Adelaide, Adelaide, Australia

$s$ Also at Department of Physics, University of Fribourg, Fribourg, Switzerland

${ }^{t}$ Also at Department of Physics, University of Michigan, Ann Arbor MI, United States of America

${ }^{u}$ Also at Dipartimento di Matematica, Informatica e Fisica, Università di Udine, Udine, Italy

$v$ Also at Faculty of Physics, M.V. Lomonosov Moscow State University, Moscow, Russia

$w$ Also at Giresun University, Faculty of Engineering, Giresun, Turkey

${ }^{x}$ Also at Graduate School of Science, Osaka University, Osaka, Japan

y Also at Hellenic Open University, Patras, Greece

$z$ Also at IJCLab, Université Paris-Saclay, CNRS/IN2P3, 91405, Orsay, France

aa Also at Institucio Catalana de Recerca i Estudis Avancats, ICREA, Barcelona, Spain

ab Also at Institut für Experimentalphysik, Universität Hamburg, Hamburg, Germany

ac Also at Institute for Mathematics, Astrophysics and Particle Physics, Radboud University Nijmegen/Nikhef, Nijmegen, Netherlands

ad Also at Institute for Nuclear Research and Nuclear Energy (INRNE) of the Bulgarian Academy of Sciences, Sofia, Bulgaria

ae Also at Institute for Particle and Nuclear Physics, Wigner Research Centre for Physics, Budapest, Hungary

af Also at Institute of Particle Physics (IPP), Vancouver, Canada

ag Also at Institute of Physics, Academia Sinica, Taipei, Taiwan

ah Also at Institute of Physics, Azerbaijan Academy of Sciences, Baku, Azerbaijan

ai Also at Institute of Theoretical Physics, Ilia State University, Tbilisi, Georgia

aj Also at Instituto de Fisica Teorica, IFT-UAM/CSIC, Madrid, Spain

ak Also at Istanbul University, Department of Physics, Istanbul, Turkey

al Also at Joint Institute for Nuclear Research, Dubna, Russia

am Also at Louisiana Tech University, Ruston LA, United States of America

an Also at LPNHE, Sorbonne Université, Université de Paris, CNRS/IN2P3, Paris, France

ao Also at Manhattan College, New York NY, United States of America

ap Also at Moscow Institute of Physics and Technology State University, Dolgoprudny, Russia

${ }^{a q}$ Also at National Research Nuclear University MEPhI, Moscow, Russia

ar Also at Physics Department, An-Najah National University, Nablus, Palestine

as Also at Physics Dept, University of South Africa, Pretoria, South Africa

at Also at Physikalisches Institut, Albert-Ludwigs-Universität Freiburg, Freiburg, Germany

au Also at School of Physics, Sun Yat-sen University, Guangzhou, China

av Also at The City College of New York, New York NY, United States of America

aw Also at The Collaborative Innovation Center of Quantum Matter (CICQM), Beijing, China

ax Also at TRIUMF, Vancouver BC, Canada

ay Also at Universita di Napoli Parthenope, Napoli, Italy

* Deceased 